Aus der Neurologischen Abteilung des Klinikums Großhadern der Ludwig-Maximilians-Universität München Prof. Dr. med. Dr. h. c. Thomas Brandt

\title{
Intima-Media-Thickness (IMT) \\ bei Patienten mit vertebrobasilären und karotidalen Stenosen/Verschlüssen
}

\author{
Dissertation \\ zum Erwerb des Doktorgrades der Medizin \\ an der Medizinischen Fakultät der \\ Ludwig-Maximilians-Universität zu München \\ 2003
}

\author{
vorgelegt von \\ Joachim Oberseider \\ geboren am 6. Oktober 1969 \\ in Neuendettelsau
}


Mit Genehmigung der Medizinischen Fakultät

der Universität München

Berichterstatter:

Mitberichterstatter:

Dekan:

Tag der mündlichen Prüfung:
Prof. Dr. G. Hamann

Prof. Dr. C. von Schacky

Prof. Dr. H. Mudra

Prof. Dr. B. Steckmeier

16. Oktober 2003 
Für meine Eltern 



\section{Inhaltsverzeichnis}

Patienten und Methoden .............................................................................9

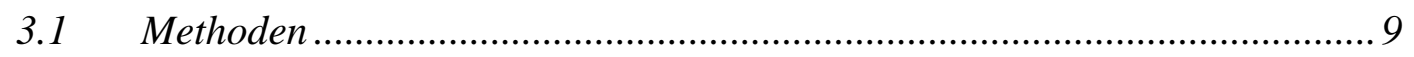

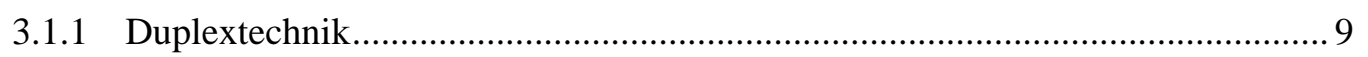

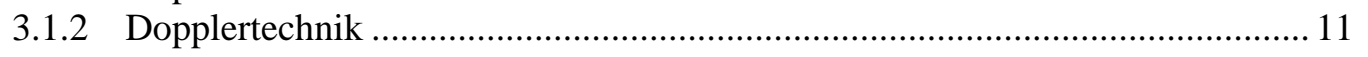

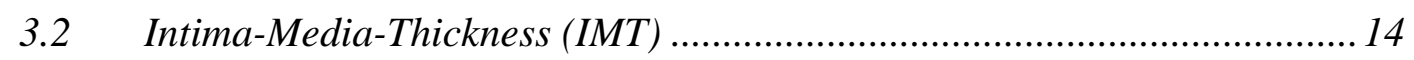

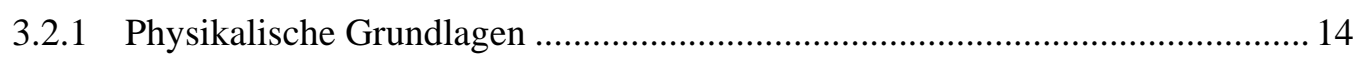

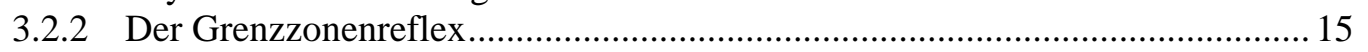

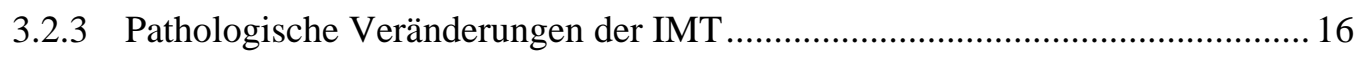

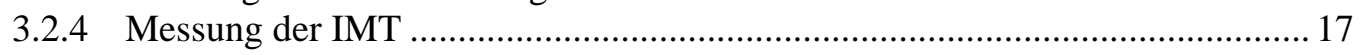

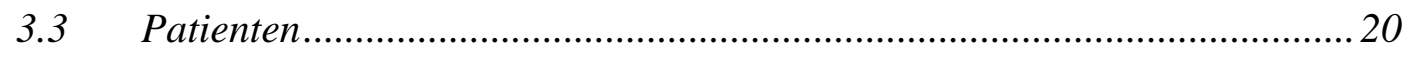

3.3.1 Stenosen/Verschlüsse im karotidalen Stromgebiet (KS) .................................. 20

3.3.2 Stenosen/Verschlüsse im vertebrobasilären Stromgebiet (VBS)........................ 21

3.3.3 Stenosen/Verschlüsse im karotidalen und vertebrobasilären Stromgebiet

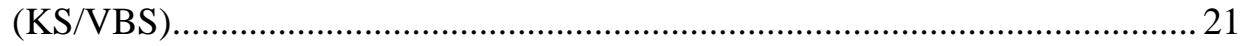

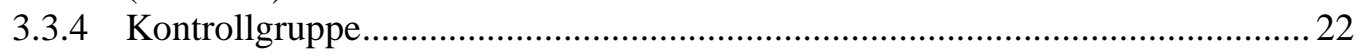

3.4 Patientenfragebogen........................................................................ 22

3.4.1 Klassische zerebrovaskuläre Risikofaktoren ........................................................ 22

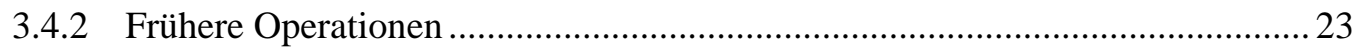

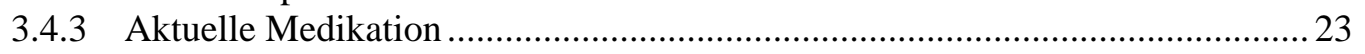

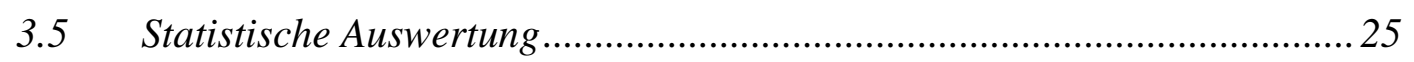

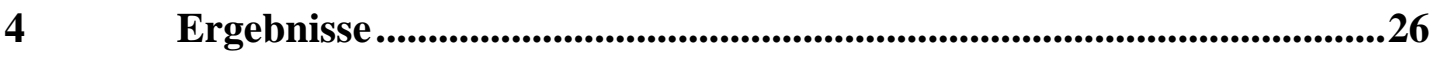

4.1 Intima-Media-Thickness bei vertebrobasilärer und karotidaler

Makroangiopathie ............................................................................... 26

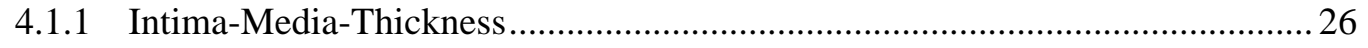

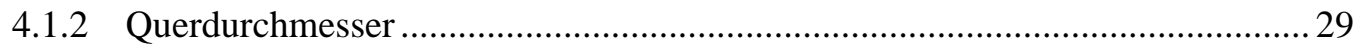

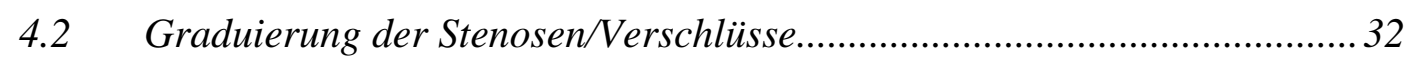

4.3 Messung der IMT der A. carotis communis (ACC) ...................................34

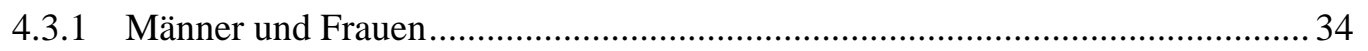

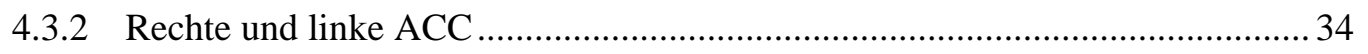


4.4 Messung des inneren Querdurchmessers (QD) der ACC ...........................36

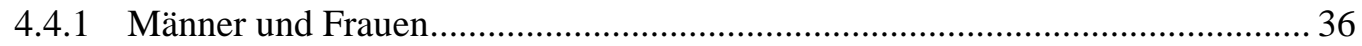

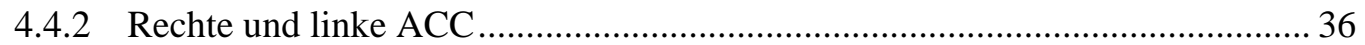

4.5 Seitenverteilung der Stenosen/Verschlüsse .............................................38

4.6 Zerebrovaskuläre Risikofaktoren.......................................................40

$5 \quad$ Diskussion 46

5.1 Intima-Media-Thickness der A. carotis communis bei vertebrobasilärer und karotidaler Makroangiopathie (Dominanztypen) ..............................46

5.2 Graduierung der Stenosen und Verschlüsse ............................................47

5.3 Zusammenhang zwischen der Intima-Media-Thickness und dem

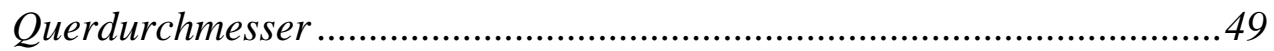

5.4 Querdurchmesser der A. carotis communis bei Männern und Frauen .......52

5.5 Intima-Media-Thickness bei Männern und Frauen ....................................54

5.6 Intima-Media-Thickness der rechten und linken A. carotis communis ......56

5.7 Seitenverteilung der Stenosen und Verschlüsse .....................................57

$5.8 \quad$ Zerebrovaskuläre Risikofaktoren.............................................................59

5.9 Methodenkritik zum Studiendesign und zur Studiengröße.........................65

$6 \quad$ Literaturverzeichnis.........................................................................6 67

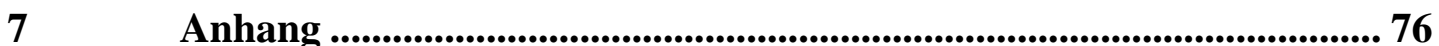

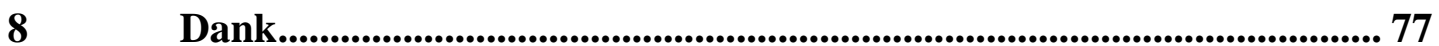

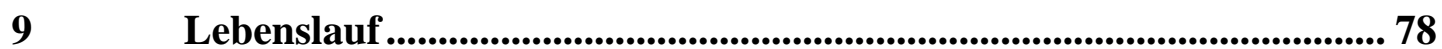




\section{Zusammenfassung}

\section{Intima-Media-Thickness (IMT) bei Patienten mit vertebrobasilären und karotidalen Stenosen/Verschlüssen}

Einleitung: Die Intima-Media-Thickness (IMT) der A. carotis communis (ACC) wird häufig als Maß für generalisierte arteriosklerotische Gefäßwandveränderungen verwendet und die Zunahme der IMT kann schon in einem frühen Stadium der Arteriosklerose im sonographischen Bild (B-mode) als Doppellinienmuster dargestellt werden. Die demographischen Unterschiede der IMT (z.B. zwischen Männern und Frauen) und die Wirkung der klassischen zerebrovaskulären Risikofaktoren auf die IMT sind hinreichend bekannt. Auch die Seitenunterschiede (rechte und linke Karotis) sowie die Prädilektionsstellen innerhalb des karotidalen Stromgebietes der IMT wurden schon beleuchtet. Unklar bleibt jedoch bisher, ob verschiedene Dominanztypen der Arteriosklerose mit einer unterschiedlichen IMT einhergehen. Untersucht wurde die IMT bei Patienten mit hämodynamisch wirksamen makroangiopathischen Veränderungen (Stenosen/Verschlüsse) des vertebrobasilären (VBS) und des karotidalen (KS) Stromgebietes.

Patienten und Methoden: Es wurden hierzu die IMT (Methode nach Touboul) und der innere Querdurchmesser (QD) der ACC (als Parameter der dilatativen Form der Arteriosklerose) beiderseits in Regionen ohne Stenosen oder deutlicher Plaquebildung gemessen. Untersucht wurden insgesamt 121 Personen, hierunter 32 Normalpersonen, 57 Patienten mit Karotisstenosen und -verschlüssen und 16 Patienten mit rein vertebrobasilären Stenosen/Verschlüssen. 16 Patienten, die sowohl vertebrobasiläre (VBS) als auch karotidale (KS) Stenosen/Verschlüsse hatten, wurden in eine vierte Gruppe (KS/VBS) eingeteilt (nach doppler- und duplexsonographischen Kriterien). Die klassischen zerebrovaskulären Risikofaktoren (Alter, Arterieller Hypertonus, Diabetes mellitus, Hypercholesterinämie, Familienanamnese, Nikotinabusus) wurden in einem Fragebogen erfaßt.

Ergebnisse: Bei Kontrollpersonen fanden sich eine IMT von 0,67 $\pm 0,1 \mathrm{~mm}$ und ein QD von $5,8 \pm 1,2 \mathrm{~mm}$. Patienten mit KS-Makroangiopathie zeigten mit 0,96 $\pm 0,2 \mathrm{~mm}$ eine signifikant erhöhte IMT $(\mathrm{p}<0,0001)$ und einen größeren QD der ACC $(6,6 \pm 1,2 \mathrm{~mm}, \mathrm{p}<0,01)$. Gegenüber den Kontrollpersonen ergaben sich auch für die Patienten mit VBS- 
Makroangiopathie eine signifikant erhöhte IMT $(0,88 \pm 0,2 \mathrm{~mm}, \mathrm{p}<0,001)$ und ein größerer QD (auf 6,9 $\pm 0,9$ mm, p<0,01). Signifikante Unterschiede zwischen den beiden Gruppen fanden sich nicht, obwohl tendenziell die IMT in der Gruppe mit Stenosen/Verschlüssen im VBS geringer erschien. Probanden ohne Makroangiopathien zeigten eine signifikant dünnere IMT $(0,67 \pm 0,08 \mathrm{~mm})$ als Patienten mit Stenosen oder Verschlüssen $(1,03 \pm 0,26$ $\mathrm{mm}, \mathrm{p}<0,001)$. Der QD korrelierte nur gering $(\mathrm{r}=0,46)$ mit der IMT.

Bei Männern wurde eine signifikant $(\mathrm{p}<0,05)$ dickere IM-Schicht mit 0,92 $\pm 0,2 \mathrm{~mm}$

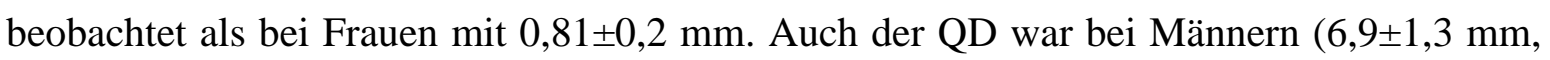
$\mathrm{p}<0,001)$ signifikant größer als bei Frauen $(5,8 \pm 1,0 \mathrm{~mm})$. Bei seitengetrennter Betrachtung (rechts/links) der IMT zeigte sich kein signifikanter Unterschied. Makroangiopathien in den hirnversorgenden Arterien waren jedoch häufiger auf der linken Seite zu finden. Hypertoniker $(0,95 \pm 0,3 \mathrm{~mm}, \mathrm{p}<0,05)$ und Diabetiker $(1,03 \pm 0,2 \mathrm{~mm}, \mathrm{p}<0,01)$ zeigten eine dickere IMT als Nicht-Hypertoniker $(0,84 \pm 0,2 \mathrm{~mm}, \mathrm{p}<0,01)$ und Nicht-Diabetiker $(0,86 \pm 0,4$ $\mathrm{mm}, \mathrm{p}<0,01)$. Bei Rauchern wurde eine dünnere IM-Schicht $(0,81 \pm 0,2 \mathrm{~mm}, \mathrm{p}<0,05)$ beobachtet als bei Nicht-Rauchern (0,92 $\pm 0,2 \mathrm{~mm})$. Raucher (57 Jahre) waren jedoch im Schnitt zehn Jahre jünger als Nicht-Raucher (67 Jahre) und das Alter korrelierte signifikant $(\mathrm{p}<0,01)$ mit der IMT.

Diskussion: Die IMT zwischen Patienten mit hämodynamischen Stenosen oder Verschlüssen im VBS und im KS unterscheidet sich im untersuchten Patientengut nicht signifikant. Patienten mit Makroangiopathien in den hirnversorgenden Arterien zeigen eine dickere IM-Schicht im Vergleich zu gefäßgesunden Probanden. Sowohl Patienten mit karotidalen als auch Patienten mit vertebrobasilären Stenosen oder Verschlüssen zeigen ein größeres Lumen (QD) der ACC im Vergleich zur Kontrollpersonen. Der QD korrelierte nur gering mit der IMT.

Unsere Ergebnisse bestätigen vorangehende Untersuchungen: Das Lumen (QD) der ACC ist bei Männern größer ist als bei Frauen und Männer zeigen eine dickere IM-Schicht als Frauen. Die IMT der rechten und linken ACC verhält sich nicht signifikant unterschiedlich. Stenosen oder Verschlüsse in den hirnversorgenden Arterien sind jedoch häufiger auf der linken Seite zu finden. Hypertoniker und Diabetiker weisen eine dickere IM-Schicht auf als Nicht-Hypertoniker und Nicht-Diabetiker. Auch das Alter korrelierte gut mit der IMT. Nicht nur die klassischen zerebrovaskulären Risikofaktoren sondern auch Faktoren wie genetische Disposition, ethnische Unterschiede, sozioökonomische Aspekte und Umwelteinflüsse können eine Zunahme der IMT bewirken. 


\section{Intima-Media-Thickness in Patients with Stenoses or Occlusions of the Carotid or the Vertebrobasilar Arteries}

Introduction: The Intima-Media-Thickness (IMT) of the commom carotid artery (CCA) is often used as a parameter for generalized atherosclerotic lesions of the vessel wall. It is possible to visualize the increase of the IMT in an early degree of athrosclerosis as a double line pattern in the sonographical picture (B-mode). The demographic difference of the IMT (e.g. between men an women) and the effect of the classical cerebrovascular risk factors on the IMT are sufficiently known. The difference of the side (right and left carotid artery) and the localisation in the carortid arteries of the IMT are already illuminated. It is not known actually, if different types of atherosclerosis are accompanied by a different IMT. The purpose of the study was to invesigate the IMT in patients with stenoses or occlusions in the vertebrobasilar system (VBS) and the carotid system (CS).

Patients and methods: The Intima-Media-Thickness (IMT) and the diameter (QD) of the common carotid artery (CCA) (as a parameter of proliferative and dilatative form of atherosclerosis) were measured on both sides on areas of vessel wall without stenoses and plaques. We examined of the neck 121 people, including 32 normal healthy counds, 57 patients with stenoses or occlusions in the CS and 16 patients with macroangiopathy exclusively in the VBS. Patients of the fourth group $(n=16)$ suffered from both VBS- and CS-macroangiopathy (CS/VBS) (following doppler- and duplex sonographical criteria).

The classical cerebrovascular risk factors (age, arterial blood pressure, hypercholesterolemia, diabetes mellitus, family history, smoking status) were entered in a questionnaire.

Results: In control persons, a IMT of $0,67 \pm 0,2 \mathrm{~mm}$ and a CCA-diameter of $5,8 \pm 1,2 \mathrm{~mm}$ was found. Patients with CS-macroangiopathy exhibited a significant higher IMT with 0,97 $\pm 0,2 \mathrm{~mm}(\mathrm{p}<0,0001)$ and a larger CCA-diameter $(6,6 \pm 1,2 \mathrm{~mm}, \mathrm{p}<0,01)$. In comparison to the controls we also found a significant higher IMT $(0,86 \pm 0,2 \mathrm{~mm}, \mathrm{p}<0,001)$ and a larger CCA-diameter $(6,8 \pm 0,9 \mathrm{~mm}, \mathrm{p}<0,01)$ in patients with VBS-macroangiopathy. There was no significant difference between both groups, despite a tendency of less severe changes in patients suffering from VBS-macroangiopathy. Persons without macroangiopthy exhibited a significant thinner $\operatorname{IMT}(0,67 \pm 0,08 \mathrm{~mm})$ than patients with stenoses and occlusions $(1,03 \pm 0,26 \mathrm{~mm}, \mathrm{p}<0,001)$. The diameter correlated only weakly $(\mathrm{r}=0,46)$ with the IMT. 
In men was found a significant thicker IMT $(0,92 \pm 0,2 \mathrm{~mm}, \mathrm{p}<0,05)$ than in women $(0,81$ $\mathrm{p}<0,050,2 \mathrm{~mm})$. Also the diameter was in men with $6,9 \pm 1,3 \mathrm{~mm}$ significantly $(\mathrm{p}<0,001)$ larger than in women with 5,8 $\pm 1,0 \mathrm{~mm}$. Considering both sides of the IMT seperately (right/ left) there was no significant difference. Macroangiopathies of the cerebral arteries were found more frequently on the left side. Patients with arterial hypertension $(0,95 \pm 0,3$ $\mathrm{mm}, \mathrm{p}<0,05)$ and patients with diabetes $(1,03 \pm 0,2 \mathrm{~mm}, \mathrm{p}<0,01)$ showed a significant thicker IMT than patients without arterial hypertension $(0,84 \pm 0,2 \mathrm{~mm}, \mathrm{p}<0,01)$ and diabetes $(0,86 \pm 0,4 \mathrm{~mm}, \mathrm{p}<0,01)$. In smokers we found a thinner $\operatorname{IMT}(0,81 \pm 0,2 \mathrm{~mm}, \mathrm{p}<0,05)$ than in non-smokers $(0,92 \pm 0,2 \mathrm{~mm})$. Smoker (57 jears) were however on average ten years younger than non-smokers and the age correlated significantly $(p<0,01)$ with the IMT.

Discussion: The IMT between patients with stenosis or occlusions in the vertebrobasilar system (VBS) and the carotid system (CS) does not differ signifcantly in the examined collective of patients. Patients with macroangiopathy exhibit a thicker IMT than normal healthy counds. Both patients with CS- and VBS-macroangiopathy show a larger diameter of the CCA in comparison to the controls.

Our results support earlier observations: The diameter of the CCA in men is larger than in women and men show a higher IMT than women. Considering both sides of the IMT seperately (right/ left) there is no difference. Macroangiopathy of the cerebral arteries is found more frequently on the left side. Patients with arterial hypertension or diabetes show a thicker IMT than patients without arterial hypertension or diabetes. The age correlates well with the IMT. Not only the classical cerebrovascular risk factors but also factors like genetic disposition, ethnic differences, socioecomomical aspects and environment may attribute to an increase in IMT. 


\section{Einleitung}

Die Arteriosklerose ist in den Industrieländern eine der häufigsten Erkrankungen. Statistisch gesehen steht sie durch ihre Folgeerscheinungen unter den Todesursachen an erster Stelle. Die arterielle Hypertonie, die Hyperlipidämie, der Diabetes mellitus, der Nikotinabusus und das Alter sind klassische zerebrovaskuläre Risikofaktoren. Sie begünstigen die Entstehung und das Voranschreiten der Arteriosklerose. Unter den Folgeerscheinungen sind neben kardialen Manifestationen vor allem zerebrovaskuläre Erkrankungen zu nennen.

Die Entstehung der Arteriosklerose geht den klinischen Symptomen oft um viele Jahre voraus. STARY et al. (1987) fanden Lipide und Schaumzellen, wahrscheinlich als ein frühes Zeichen der Arteriosklerose, schon in Arterien von Kindern. Sie dokumentierten eine erhöhte Anzahl arteriosklerotischer Läsionen bei jungen Soldaten, die im Kampf gefallen waren. Die Arterioskleroseprogression bleibt solange klinisch stumm bis die Läsionen einen irreversiblen Endzustand erreicht haben (z.B. Lumeneinengung, Verkalkung und Nekrose). Deshalb ist es eine Herausforderung frühe Manifestationen der Arteriosklerose wie z.B. die Zunahme der IMT rechtzeitig zu erkennen.

Die Angiographie ist eine sehr genaue Untersuchungsmethode um die Morphologie der Karotiden darzustellen. Obwohl sie möglicherweise nichtstenotische ulzerative arteriosklerotische Veränderungen bei einigen Patienten nicht erkennt, demonstriert die Karotisangiographie Stenosen, intraluminale Thromben und extrakranielle sowie intrakranielle Gefäßverschlüsse mit höchster Präzision. Aufgrund der Risiken (invasive Methode) und der Kosten einer Angiographie, sind nicht-invasive Untersuchungsmethoden zur Darstellung der Karotiden bei asymptomatischen Patienten mit zerebrovaskolärem Risikopotential oder bei Patienten mit asymptomatischen Karotisläsionen entwickelt worden.

Seit dem Ende der 80er Jahre ist es möglich mit hochauflösenden Ultraschallgeräten, die Wanddicke arterieller Gefäße zu messen [POLI 88, PIGNOLI 86]. Zahlreiche Studien zeigten Korrelationen klassischer zerebrovaskulärer Risikofaktoren mit der IMT der A. carotis communis (ACC), so daß eine hier nachweisbare Verdickung als ein frühes Stadium 
der Arteriosklerose angesehen werden kann [GROBBEE 94, HEISS 91, SALONEN R 91, BOTS 97, O'LEARY 92, OBERSEIDER 99].

Schon 1982 erkannten JAMES et al., daß bei sonographischer Darstellung der Arterienwand zwei parallele echoreiche und eine echoarme Schicht sichtbar werden. In dieser Studie zeigte sich eine gute Korrelation zwischen angiographischer und sonographischer Bildgebung, wenn man das Ausmaß der Plaquemorphologie beurteilte.

TERWEY et al. beschrieben 1984 die charakteristische Echostruktur (Doppellinienmuster) als physikalisches Artefakt, den sogenannten "Grenzzonenreflex", welcher durch Reflexion der Schallwellen an den Grenzflächen zweier unterschiedlicher Strukturen (z.B. Blut und Intima) entsteht. Sie lancierten die Hypothese, "daß es sich bei diesen Strukturen um die histologisch differenzierbaren Wandschichten Intima, Media und Adventitia handeln könne."

Die Validität der IMT-Messung stützt sich auf die Ergebnisse von PIGNOLI et al., welche 1986 als erste einen Vergleich zwischen in vitro und in vivo Messung der IMT der ACC anstellten. Dabei zeigte sich, daß die Dicke von Intima plus Media sonographisch (BMode) mit der Dicke von Intima plus Media am Obduktionsresektat weitgehend übereinstimmte. Es ergab sich kein signifikanter Unterschied der IMT.

Die erste computer-gesteuerte Messung der IMT in der ACC wurde von POLI et al. (1988) in einer Studie mit Patienten, die an Hypercholesterinämie litten, durchgeführt. Sie fanden eine signifikante Verdickung des Intima-Media-Komplexes (IM-Komplex) im Vergleich zu Gefäßgesunden und postulierten die IMT als Screeningparameter zur Früherkennung und Verlaufskontrolle der Arteriosklerose.

Seitdem sind zahlreiche epidemiologische und klinische Interventionsstudien [CROUSE 94, CROUSE 96, BOND 91, CHAMBLESS 96, HOWARD 93] abgeschlossen worden oder gerade in Bearbeitung, welche die IMT der extrakraniellen karotidalen Arterien gemessen mit hochauflösenden Ultraschall als prognostischen Marker für Arteriosklerose einsetzen.

Prädilektionsstellen der zerebrovaskulären Arteriosklerose sind die Arteria carotis interna (ACI) sowie die Karotisifurkation (Bif.) [ZARINS 83, SITZER 03]. Stenosen oder 
Verschlüsse dieser Gefäße sowie Embolisationen aus diesem Bereich können zu zerebralen Ischämien führen.

Wandverdickungen der ACC (IMT) lassen auch Rückschlüsse auf das Stadium der Arteriosklerose in anderen Gefäßregionen zu, wie Aorta, Koronar- und Femoralarterien [GROBBEE 94, SALONEN 93, STARY 92, GEROULAKOS 94, VELLER 93].

Die IMT in der distalen ACC nimmt schon in einem frühen Stadium der Arteriosklerose zu [PERSSON 94] und kann möglicherweise als prognostischer Faktor für zukünftige arteriosklerotische Komplikationen dienen. So hatten z.B. Patienten mit einer Zunahme der IMT ein erhöhtes Risiko für koronare Ischämien [HODIS 98].

Häufig wurden die demographischen Unterschiede der IMT dargestellt. So ist bekannt, daß die IMT mit dem Alter kontinuierlich zunimmt [ROSFORS 98, FABRIS 94] und Männer eine dickere IM-Schicht aufweisen als Frauen [BOTS 97, MANNAMI 00].

HOWARD et al. (1994) unterschieden verschiedene Formen der Arteriosklerose. Die gleichmäßige Wirkung der zerebrovaskulären Risikofaktoren auf das gesamte arterielle Gefäßsystem hob die systemische Form der Arteriosklerose hervor. Die anatomische Form der Arteriosklerose wurde durch die bevorzugte Enstehung von Plaques/Stenosen (Prädilektionsstellen) wie z.B. in und oberhalb der Karotisbifurkation deutlich.

Auch die Seitenpräferenz von Makroangiopathien deutet auf eine anatomische Form der Arteriosklerose hin. Stenosen und Verschlüsse waren im karotidalen Stromgebiet häufiger links lokalisiert [ROSFORS 98].

Unklar bleibt jedoch bisher, ob verschiedene Dominanztypen der Arteriosklerose mit einer unterschiedlichen allgemeinen Arterioskleroseausprägung (IMT) einhergehen. In unserer Studie werden zwei Dominanztypen unterschieden: Patienten mit rein vertebrobasilären und Patienten mit rein karotidalen Stenosen und Verschlüssen. Untersucht wurde hierzu die IMT bei Patienten mit hämodynamisch wirksamen makroangiopathischen Veränderungen (Stenosen/Verschlüsse) des vertebrobasilären (VBS) und des karotidalen (KS) Stromgebietes. 
Die Fragestellungen und Ziele unserer Studie lassen sich wie folgt zusammenfassen:

1) Gibt es signifikante Unterschiede der Intima-Media-Thickness (IMT) bzw. des inneren Querdurchmessers (QD), wenn man gesunde Probanden und Patienten mit Makroangiopathien vergleicht?

2) Gibt es signifikante Unterschiede der IMT bei verschiedenen Dominanztypen der Arteriosklerose wie rein vertebrobasiläre und rein karotidale Stenosen und Verschlüssen?

3) Findet man bei Patienten mit mehr als einer Stenose/Verschluß der hirnversorgenden Arterien eine signifikant größere IMT als bei Patienten mit nur einer Stenose/Verschluß?

4) Besteht eine signifikante Korrelation zwischen der IMT und dem QD?

5) Gibt es signifikante Unterschiede des QD zwischen Männern und Frauen?

6) Gibt es signifikante Unterschiede der IMT zwischen Männern und Frauen?

7) Gibt es signifikante Unterschiede der IMT, wenn man die rechte und linke Seite vergleicht?

8) Gibt es bei Stenosen/Verschlüssen der hirnversorgenden Arterien eine Seitenbevorzugung zwischen linker und rechter Seite?

9) Wie verhält sich die IMT, wenn man Patienten mit zerebrovaskulären Risikofaktoren (Arterieller Hypertonus, Diabetes mellitus, Hypercholesterinämie, Nikotinabusus usw.) und Normalpersonen vergleicht?

10) Besteht eine signifikante Korrelation zwischen dem Alter und der IMT bzw. des QD?

11) Gibt es einen Zusammenhang zwischen vorangehenden zerebralen bzw. kardialen Ereignissen (zerebrale oder kardiale Ischämie) und der IMT? 


\section{Patienten und Methoden}

\subsection{Methoden}

\subsubsection{Duplextechnik}

Alle Untersuchungen wurden mit einem Duplexgerät durchgeführt. Die Duplexsonographie ist die sinnvolle Verknüpfung von Doppler- und Schnittbildsonographie. Der besondere Vorteil der Duplexsonographie liegt in der Möglichkeit der simultanen Beurteilung von Gefäßmorphologie und Blutfluß. Die Schnittbildsonographie (B-mode-Technik) ist ein Verfahren zur Signalverarbeitung von Ultraschall-Echos, bei dem die Signale als Punkte intensitätsproportionaler Helligkeit („,brightness“) auf der Nullinie eines Oszillographen abstandsproportional wiedergegeben werden. Mehrere B-mode-Linien können zu zweidimensionalen B-Bildern kombiniert werden. Die Darstellung der A. carotis communis im Longitudinal- und Transversalschnitt mit B-mode-Technik war die Voraussetzung zur Messung der IMT.

Anfangs wurde die Duplextechnik durch Installation von Schnittbildschallkopf und Dopplersonde in einem Gehäuse realisiert. Heute verwendet man dieselben Schallwandler für beide Techniken. Je nach Verfahren zur Bewegung der akustischen Achse wird zwischen mechanisch und elektronisch gesteuerten Wandlern unterschieden. Elektronisch gesteuerte Wandler (phased-array-transducer) bieten demgegenüber die Möglichkeit, einen „virtuellen“ Schallstrahl relativ beliebig im Bildausschnitt zu positionieren. Das ist von großem Vorteil, wenn der Schallstrahl (wie bei mechanisch gesteuerten Tranducern z.B. Sektorschallköpfe) vom Zentrum des ausgeschnittenen Sektors ausgeht. Dann entsteht nämlich zu Gefäßen, die parallel zur Hautoberfläche verlaufen (z.B. ACC), ein senkrechter Winkel.

Anhand eines drehbaren Balkens ist es möglich den „Beschallungswinkel“ (Cosinusfunktion in der Dopplergleichung) durch Winkelkorrektur zu optimieren und somit die Messungen zu standardisieren. Es ist nun naheliegend, jetzt nicht nur Aussagen über Dopplerfrequenzverschiebungen zu machen, sondern auch Strömungsgeschwindigkeiten $(\mathrm{cm} / \mathrm{s})$ zu messen. Es ist jedoch darauf zu achten, daß Winkel von $70^{\circ}$ und mehr vermieden 
werden, da sonst inakzeptable Meßfehler auftreten können. Zwei wesentliche Vorteile der winkelkorrigierten Messung seien nachfolgend erwähnt:

1. Zum einen sind auch bei Gefäßanomalien (z.B. Knick- und Schlingenbildung) zuverlässige Untersuchungen möglich.

2. Zum anderen liefern die winkelkorrigierten Messungen Ergebnisse besserer Reproduzierbarkeit.

Die Berechnung des Volumenflusses I erfolgt bei Kenntnis der Strömungsgeschwindigkeit $\mathrm{v}_{\mathrm{m}}$ und des Gefäßquerschnitts F nach der Gleichung:

$$
I=F * v_{m}
$$

Die Bestimmung des Volumenflusses ist z.B. bei arteriovenösen Fisteln von Bedeutung. Durch Messung des Shuntvolumens kann das Ausmaß der kardialen Belastung beurteilt werden. Eine korrekte Messung erhält man nur dann, wenn das Meßvolumen ausreichend groß ist und das Gefäß im Longitudinalschnitt auf wenigstens 1-2 cm dargestellt wird. Zur Berechnung wird die arithmetisch gemittelte Strömungsgeschwindigkeit „Mean-Wert“ herangezogen:

$$
v_{m}=1 / 3\left(v_{s}+2 v_{d}\right)
$$

$\mathrm{v}_{\mathrm{m}}=$ Mean-Wert der Strömungsgeschwindigkeit

$\mathrm{v}_{\mathrm{s}}=$ maximale systolische Strömungsgeschwindigkeit

$\mathrm{v}_{\mathrm{d}}=$ maximale enddiastolische Strömungsgeschwindigkeit

Besonders bei der Schnittbildsonographie sind biologisch bedingte Fehlerquellen stets zu berücksichtigen. Wiederholechos erscheinen immer dann, wenn sich zwei starke Reflektoren parallel gegenüberstehen. Der Ultraschallimpuls wird hin und her geschleudert. Der dadurch entstehende „Ping-Pong-Effekt“ sorgt für störende Artefakte auf dem Bildschirm. 
Durch echoreiche, oft kalkhaltige Gefäßwandanteile werden (in Schallausbreitungsrichtung) dahinter befindliche Strukturen ausgelöscht oder undeutlich wiedergegeben. Die dadurch entstehenden Schallschatten sind ein häufiges Problem bei der Beurteilung von Gefäßstenosen.

Echoreiche Plaques an der lateralen Gefäßwand (exzentrische Stenosen) projizieren sich aufgrund der eingeschränkten seitlichen Auflösung der Schallwandler zuweilen in die Schnittebene. Dies kann zu erheblichen Fehleinschätzungen des Stenosegrades führen. Deshalb ist es wichtig Gefäße in möglichst vielen Ebenen darzustellen.

\subsubsection{Dopplertechnik}

In allen Untersuchungen mit dem Duplexscan wurde das gepulste Dopplerverfahren (pwDoppler) mit der Schnittbildsonographie verknüpft. Dies bedeutet, daß das Gerät nicht wie die kontinuierlich sendenden Doppler (cw-Doppler) ständig Ultraschallwellen sendet und empfängt, sondern der Schallkopf abwechselnd kurz Sendeimpulse abgibt und in der Zeit zwischen den Sendeimpulsen als Empfänger wirkt. Die Sendezeit (einige $\mu s$ ) ist im Vergleich zu der für den Empfang zur Verfügung stehenden Zeit relativ kurz. Die Laufzeit des Sendeimpulses zu einem bewegten Reflektor bzw. Zerstreuer, im Falle des Gefäßes also die korpuskulären Bestandteile des Bluts, und zurück, ist ein Maß für die Entfernung desselben von der Sonde bzw. der Annäherung des Zerstreuers auf die Sonde zu. Je größer die Fließgeschwindigkeit dieser Körperchen ist, desto größer ist die Differenz zwischen gesendeter und zurückgestreuter Frequenz, die sogenannte Doppler-Verschiebefrequenz. Die Berechnung dieser Frequenz erfolgt sowohl für die Verfahren mit kontinuierlicher als auch mit gepulster Schallaussendung nach der Gleichung:

$$
\Delta f=\frac{2 * v * f_{0} * \cos \alpha}{c}
$$

$\mathrm{v}=$ Blutströmungsgeschwindigkeit $(\mathrm{cm} / \mathrm{s})$

$\mathrm{f}_{0}=$ Mittelwert der Sendefrequenz $(\mathrm{Hz})$

$\cos \alpha=$ Beschallungswinkel

$\mathrm{c}=$ Schallgeschwindigkeit im Gewebe $(\mathrm{cm} / \mathrm{s})$

$\Delta \mathrm{f}=$ Freqenzverschiebung in $\mathrm{Hz}$ (Dopplefrequenz) 
Aufgelöst nach der Geschwindigkeit der Blutkörperchen ergibt sich also

$$
v=\frac{\Delta f * c}{2 f_{0} * \cos \alpha}
$$

Hieraus wird die Wichtigkeit des Beschallungswinkels $\alpha$ deutlich. Da nur die Relativbewegung eines Reflektors, bezogen auf die Beschallungsrichtung, gemessen wird, wird die Strömungsgeschwindigkeit nur dann 100\%ig wiedergegeben, wenn die Bewegung des Reflektors und die Schallausbreitung gleichgerichtet sind (Cosinus=1). Bei einer Beschallung im rechten Winkel dagegen kann keine Geschwindigkeitsmessung erfolgen (Cosinus=0). Die Messung der höchstmöglichen Dopplerfrequenz bedeutet also immer, daß der kleinstmögliche Beschallungswinkel gewählt wurde, was sich auf die Reproduzierberkeit bereits gewonnener Ergebnisse positiv auswirkt. Der Meßfehler der Dopplerfrequenz bleibt um so kleiner, je flacher der Beschallungswinkel gewählt wird, wobei zusätzlich die Frequenz der gewählten Sonde zu berücksichtigen ist. Die folgende Tabelle verdeutlicht dies für eine $2 \mathrm{MHz}$ Sonde (Tab. 1). 
Tab. 1 Meßfehler der Dopplerfrequenz bei Berücksichtigung der Blutflußgeschwindigkeit und des Beschallungswinkels $\cos \alpha$

\begin{tabular}{|c|c|c|}
\hline $\begin{array}{c}\text { Blutgeschwindigkeit in } \\
\mathrm{cm} / \mathrm{s}\end{array}$ & $\begin{array}{c}\text { Beschallungswinkel cos } \alpha \\
\text { (Meßgenauigkeit der } \\
\text { Dopplerfrequenz in \%) }\end{array}$ & $\begin{array}{c}\text { Dopplerfrequenz in } \\
\mathbf{k H z}\end{array}$ \\
\hline 50 & $0(1,0)$ & 1,3 \\
\hline 50 & $30(0,87)$ & 1,1 \\
\hline 50 & $45(0,71)$ & 0,9 \\
\hline 50 & $60(0,50)$ & 0,6 \\
\hline 50 & $90(0)$ & 0 \\
\hline
\end{tabular}

Beschallungswinkel $\cos \alpha=$ Winkel zwischen Strömungsrichtung des Blutes und der Schallausbreitungsrichtung, Beschallungswinkel $0^{\circ}=100 \%$ ige Wiedergabe der Strömungsgeschwindigkeit $=$ Blutfluß und Schallausbreitung sind gleichgerichtet

Der entstehende Fehler bei einem Beschallungswinkel von $60^{\circ}$ beträgt also ca. $50 \%$.

Mittels einer Torschaltung, welche den Empfang nur innerhalb fester Zeitgrenzen ermöglicht, ist eine Festlegung der Beschallungsgrenzen zu erreichen. Dabei beeinflußt der zeitliche Abstand zwischen Impuls und Beginn des Empfangs im wesentlichen die Beschallungstiefe, die Dauer der Toröffnung und vor allem die räumliche Ausdehnung des Meßvolumens. Der Vorteil des gepulsten Dopplers im Gegensatz zum kontinuierlichen Doppler besteht darin, daß er in einem wählbaren Tiefenbereich die überlagerungsfreie Messung der Fließgeschwindigkeit gewährleistet und somit Einflüsse anderer Gefäße, die in der gleichen Schallkeule liegen, ausgeschlossen werden.

Dennoch beinhaltet der gepulste Doppler einen Nachteil. Ist die Entfernung des Sendeelements vom Reflektor groß, so sinkt die mögliche Pulsrepetitionsfrequenz (PRF) des Senders, da der Weg des Echos der Distanz gemäß längere Zeit benötigt, um den Empfänger zu erreichen. Die PRF bestimmt die höchste meßbare Dopplerfrequenz. Diese 
entspricht der halben PRF, der sogennanten Nyquist-Frequenz. Liegt nun die zu messende Dopplerfrequenz deutlich über der PRF, wird eine zu geringe Frequenz gemessen. Bei langen Laufzeiten der Echos kann es also schwierig sein, mit gepulster Dopplersonographie hohe Frequenzen, wie sie unter anderem bei Stenosen vorkommen, zu messen. Dieses Problem stellt sich jedoch vor allem bei der Darstellung abdomineller oder kardialer Gefäße und spielt bezüglich des extrakraniellen Dopplers der Halsgefäße (ACC) eine untergeordnete Rolle.

Die zu verwendende Ultraschallfrequenz des Senders wird durch die anatomische Lage des zu untersuchenden Gefäßes sowie durch den Schallwiderstand desjenigen Gewebes bestimmt, das zwischen Sender und Gefäß durchdrungen werden muß. Je größer die erforderliche Eindringtiefe und je schalldichter das dazwischen liegende Gewebe ist, desto niedriger muß die Sendefrequenz gewählt werden. Im Falle der extrakraniellen Dopplersonographie der ACC kommen 4-5 MHz Sonden zum Einsatz.

\subsection{Intima-Media-Thickness (IMT)}

\subsubsection{Physikalische Grundlagen}

\section{Reflexion}

Der Grenzzonenreflex [TERWEY 83, WIDDER 95] entsteht durch Reflexion der Schallwellen an Grenzflächen zweier unterschiedlicher Strukturen des menschlichen Körpers (z.B. Blut und Intima). Denn jede dieser Strukturen besitzt einen spezifischen Schallwiderstand, der in der Physik als Impedanz bezeichnet wird. Je größer dieser Impedanzunterschied ist, desto stärker imponiert die Reflexion. Die Reflexion gemäß der optischen Gesetze kann nur dann beobachtet werden, wenn die Grenzfläche annähernd senkrecht zum Schallstrahl steht und die Größe des untersuchten Gewebes mindestens der Wellenlänge des Ultraschalls entspricht (0,2-1mm). Der Impedanzsprung zwischen Blut und Gewebe ist groß, so daß sich die Gefäßwand durch eine Reflexion an der Außen- und Innenfläche darstellt. 


\section{Auflösung}

Das Auflösungsvermögen eines Ultraschallgerätes ist für die exakte Darstellung der Grenzflächen von großer Bedeutung [WIDDER 95]. Auch die Gefäßwanddicke, der Beschallungswinkel und der Schallwiderstand des davorliegeneden Gewebes beeinflussen die Wiedergabequalität.

Das Auflösungsvermögen ist der kleinste Abstand zweier Punkte (Reflektoren) im Gewebe, die noch getrennt voneinander dargestellt werden können. Die axiale Auflösung stellt die in Schallausbreitungsrichtung angeordneten Punkte dar und ist direkt proportional zur Wellenlänge des erzeugten Ultraschalls d.h. die Auflösung ist um so besser je kürzer die Wellenlänge ist. Hochfrequente Wellen haben aber eine geringere Eindringtiefe d.h. desto kürzer die Wellenlänge desto geringer kann der Ultraschall ins Gewebe eindringen.

Die ideale Einstellung ist also immer ein Kompromiß zwischen Auflösungsvermögen und Eindringtiefe. Da die ACC in der Regel nur wenige Millimeter unter der Hautoberfläche verläuft, konnte der Kompromiß in dieser Studie zugunsten des Auflösungsvermögens geschlossen werden. Im besten Fall ist eine axiale Auflösung gleich der Wellenlänge zu erreichen. In Abhängigkeit zur Sendefrequenz beträgt diese dann zwischen 0,2 und 1mm. RILEY et al. (1992) zeigten, daß eine Änderung der IMT von kleiner 0,1 mm meßbar ist.

Zusammenhang zwischen der Wellenlänge $\lambda$ und Frequenz f:

$$
\lambda=\mathrm{c} / \mathrm{f} \quad \mathrm{c}=\text { Schallgeschwindigkeit }
$$

(ca. 1500 m/s im Körpergewebe)

\subsubsection{Der Grenzzonenreflex}

Die Grenzen zwischen den verschiedenen Gefäßwandschichten werden mit der hochauflösenden B-Mode Sonographie sowohl in der gesunden als auch in der erkrankten Arterie dargestellt. Diese Grenzen erscheinen im Longitudinalschnitt als zwei parallele echoreiche Linien, die von einer echoarmen Zwischenschicht getrennt werden. Diese charakteristische Echostuktur ist ein physikalisches Artefakt, der sogenannte "Grenzzonenreflex" [TERWEY 84]. 
Das Doppellinienmuster kann in der schallkopfnahen und -fernen Wand beobachtet werden Das echoarme Lumen der Arterie dient als akustisches Fenster und bildet das Doppellinienmuster der schallkopffernen Wand deutlicher ab. Die erste echoreiche Linie der schallkopffernen Wand entspricht der Grenzfläche zwischen Lumen und Intima (L-I) während die zweite echoreiche Linie die Media-Adventitia-Grenzfläche (M-A) repräsentiert (Abb. 1).

Abb. 1 Intima-Media-Thickness (IMT) gemessen in der A. carotis communis (ACC) $>0,8 \mathrm{~mm}$ bei einem Patienten mit fortgeschrittener Arteriosklerose
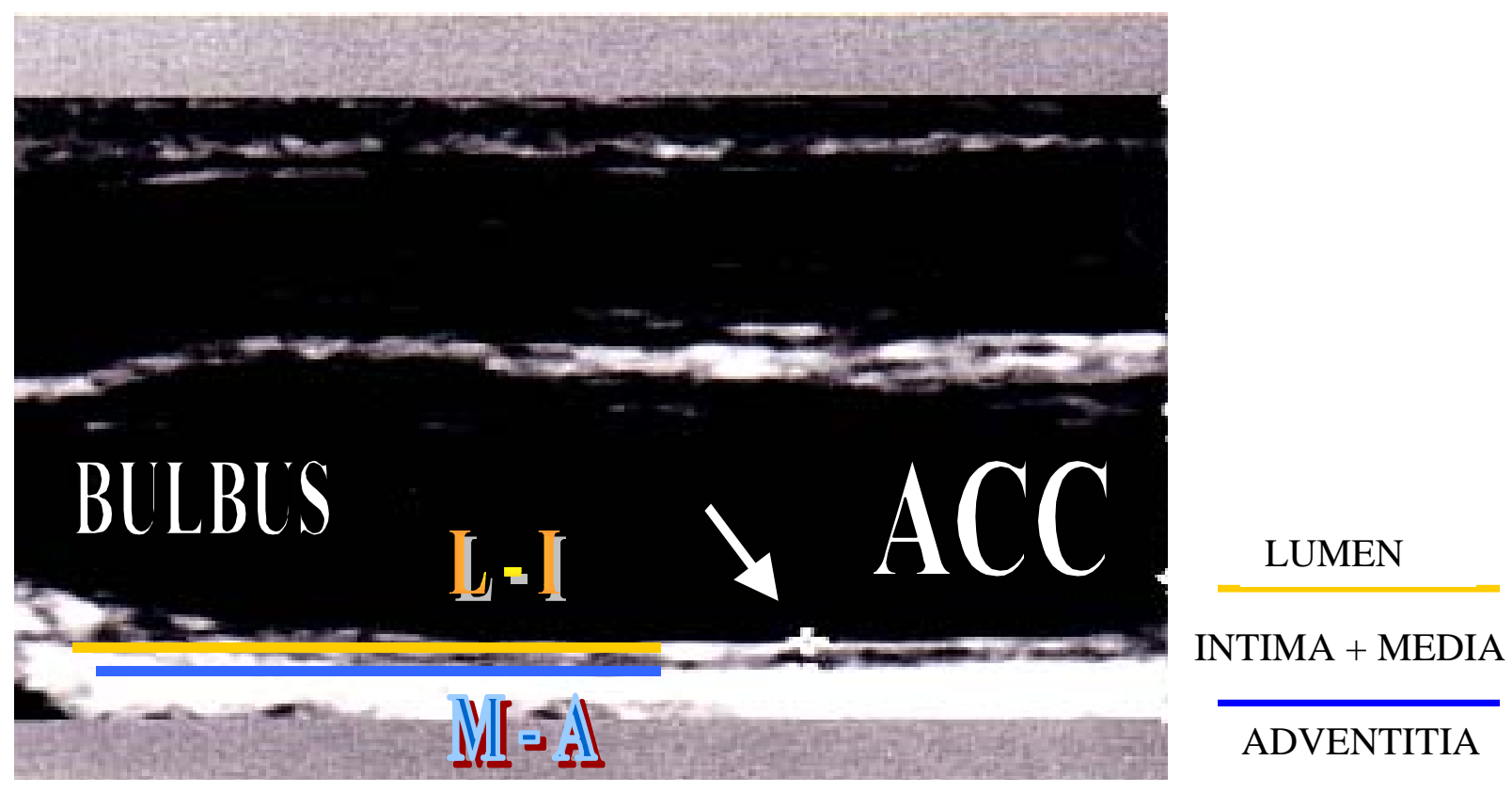

ADVENTITIA

$\downarrow$ Messung der IMT in der ACC $1 \mathrm{~cm}$ proximal des Flußteilers,

L-I = Lumen-Intima-Grenzschicht, $\mathrm{M}-\mathrm{A}$ = Media-Adventitia-Grenzschicht, ACC $=$ A. carotis communis, Bulbus $=$ Flußteiler der ACC

Die IMT ist die Distanz zwischen den zwei parallelen echoreichen Linien, wobei die Media den größten Anteil dieses Komplexes einnimmt [TOUBOUL 92]. JAMES et al. waren die ersten, die dieses Phänomen 1982 beschrieben [JAMES 82]. PIGNOLI et al. (1986) untersuchten die ACC histologisch um zu beweisen, daß die Distanz zwischen diesen beiden Linien mit der Dicke von Intima plus Media (IMT) in etwa übereinstimmt.

\subsubsection{Pathologische Veränderungen der IMT}

Eine IMT mit Werten größer $1 \mathrm{~mm}$ ist stets als pathologisch zu betrachten, insbesondere bei jungen Menschen mit Normalwerten von 0,5 mm [RUBBA 94]. Bei älteren Menschen 
wird eine IMT von 0,7-0,8 mm als physiologisch angesehen [LUDWIG 89, LUDWIG U. STUMPE 94].

Abb. 2 Intima-Media-Thickness (IMT) gemessen in der A. carotis communis (ACC) $<0,8 \mathrm{~mm}$ bei einem gesunden Probanden

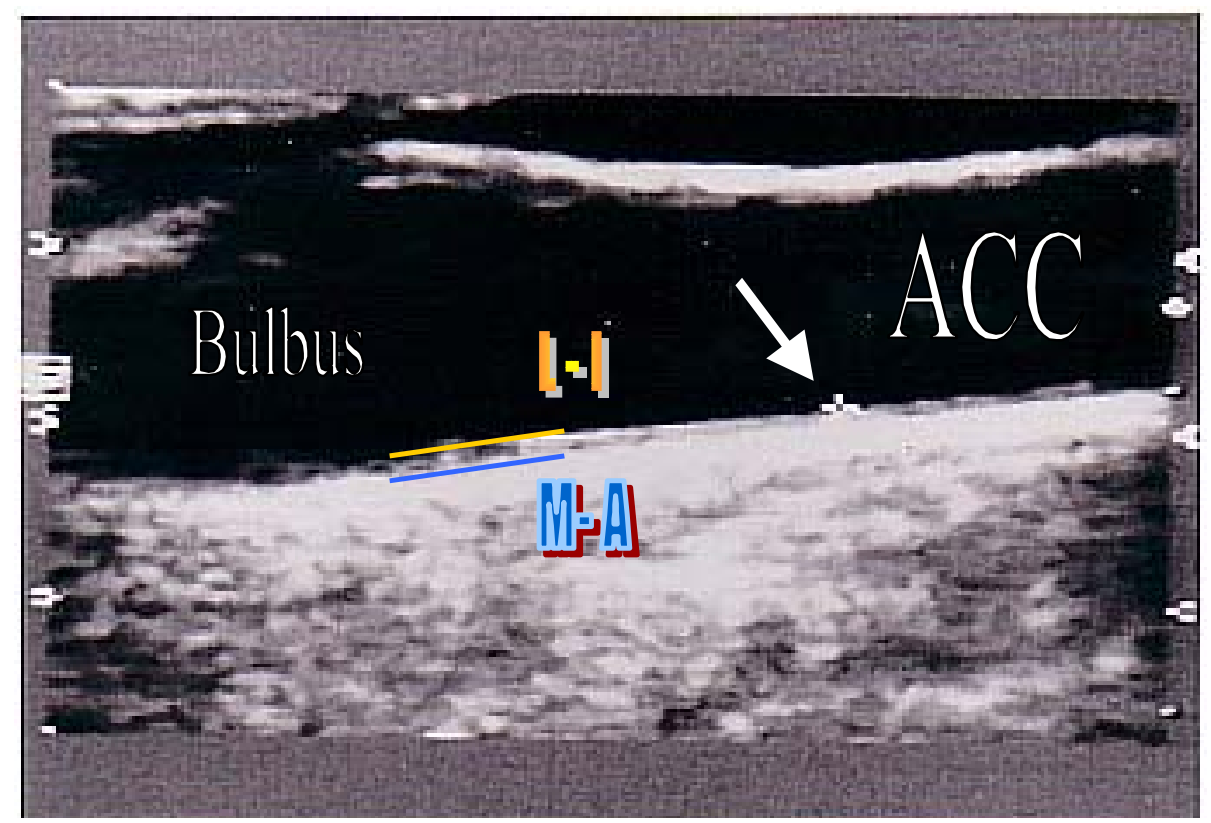

\footnotetext{
Messung der IMT in der ACC $1 \mathrm{~cm}$ proximal des Flußteilers, L-I = Lumen-Intima-Grenzschicht, M-A = Media-Adventitia-Grenzschicht, ACC $=$ A. carotis communis, Bulbus $=$ Flußteiler der ACC
}

$\mathrm{Zu}$ unterscheiden sind diffuse generalisierte Gefäßwandveränderungen von fokal anatomischen. Während bei der generalisierten Gefäßwandverdickung die IMT durchgehend vergrößert ist, tritt die fokale Form herdförmig begrenzt auf, wie z.B. bei Plaques und Stenosen. Die Echodichte der Plaques und Stenosen ist variabel und häufig inhomogen. Echoreiche generalisierte Gefäßwandverdickungen sind typisch bei Fettstoffwechselstörungen oder Diabetes mellitus. Findet man eine echoarme generalisierte Wandverdickung, muß man auch an einen entzündlichen Prozeß denken (z.B. Vaskulitis).

\subsubsection{Messung der IMT}

Wir wählten die Methode nach Touboul. Die IMT der ACC wurde auf der rechten und linken Seite immer $1 \mathrm{~cm}$ proximal des Flußteilers gemessen. Es wurde ausschließlich die 
schallkopfferne Wand zur IMT-Messung herangezogen. Deutlich erkennbare Plaques wurden nicht in die Messung miteinbezogen. IMT-Messungen wurden nur dann gewertet, wenn die Lumen-Intima-Grenzschicht gerade und in etwa parallel zur Adventitia verlief [TOUBOUL 92]. Die Messungen wurden mit einem Duplexgerät Sonos 2000 von HP durchgeführt. Das Gerät war mit einem 7,5 MHz Trapez-Linear Schallkopf ausgestattet.

WONG et al. (1993) zeigten, daß bei Messung der schallkopfnahen IMT die Werte um etwa zwanzig Prozent niedriger lagen als bei der exakten histologischen Messung am Karotisresektat. Bei den Messungen der schallkopffernen Wand stimmten die Ergebnisse mit der Histologie überein [WONG 93, PIGNOLI 86]. Um Meßfehler zu vermeiden wurde in unserer Studie nur die IMT der schallkopffernen Wand bestimmt [WIKSTRAND 94, WENDELHAG 92].

Die IMT wurde auf beiden Seiten jeweils in zwei Ebenen (anterolateral/lateral) gemessen [BLANKENHORN 93]. Wir wählten diese Methode wegen der leichten Durchführbarkeit und der guten Reproduzierbarkeit. Der Vergleich aller im Zeitraum von 1991 bis einschließlich 1995 veröffentlichten Methoden zur in vivo Messung der IMT in der ACC zeigten, daß die beste Reproduzierbarkeit gegeben war, wenn die IMT in mehr als einer Ebene gemessen wurde [KANTERS 97].

Die IMT wurde stets während der Diastole bestimmt. FROST et al. (1998) fanden eine negative Korrelation zwischen dem aktuellen Blutdruckwert und der IMT. Wegen der verläßlicheren Messung, an der während der Diastole etwas dickeren Gefäßwand, empfiehlt sich die IMT-Bestimmung zu diesem Zeitpunkt.

Bei allen untersuchten Patienten und Probanden wurde die ACC beiderseits sowohl im Longitudinal- als auch im Transversalschnitt im Zoom-Modus (2,5fache Vergrößerung) dargestellt. Bei den Longitudinalschnitten wurden zwei Ebenen (anterolateral/lateral) beschallt. Diese (insgesamt sechs Messungen pro Teilnehmer) wurden digital eingefroren und auf Video gespeichert.

In einer zweiten Sitzung (off-line analysis) wurde dann die IMT nach den Prinzipien der "leading-edge" - Methode [WIKSTRAND 94, WENDELHAG 92] gemessen. Der "leadingedge" ist die obere Demarkationslinie eines Echos (in Schallausbreitungsrichtung). Die Messung wurde manuell mit elektronisch gesetzten Markern durchgeführt und mit einem 
integrierten Meßprogramm berechnet [WENDELHAG 92, O'LEARY 92, PERSSON 94]. Jede Messung wurde dokumentiert (Video Graphic Printer, Sony).

Im Tranversalschnitt wurde zusätzlich der innere Querdurchmesser (QD) der ACC ermittelt. Der QD wurde als der Abstand zwischen Lumen-Intima-Grenzschicht der schallkopfnahen Wand und der Lumen-Intima-Grenzschicht der schallkopffernen Wand definiert [WIKSTRAND 94]. Auch der QD wurde nach den Prinzipien der "leading-edge" Methode bestimmt.

Die Untersuchungen wurden in einem licht- und geräuscharmen Raum durchgeführt. Dabei lagen die Patienten in Rückenlage bequem auf einem gepolsterten Kipptisch bei etwa $60^{\circ}$ mit dem Hals nach hinten rechts bzw. links überstreckt (Abb. 3).

Abb. 3 Situation einer Patientin während der doppler- und duplexsonographischen Untersuchung

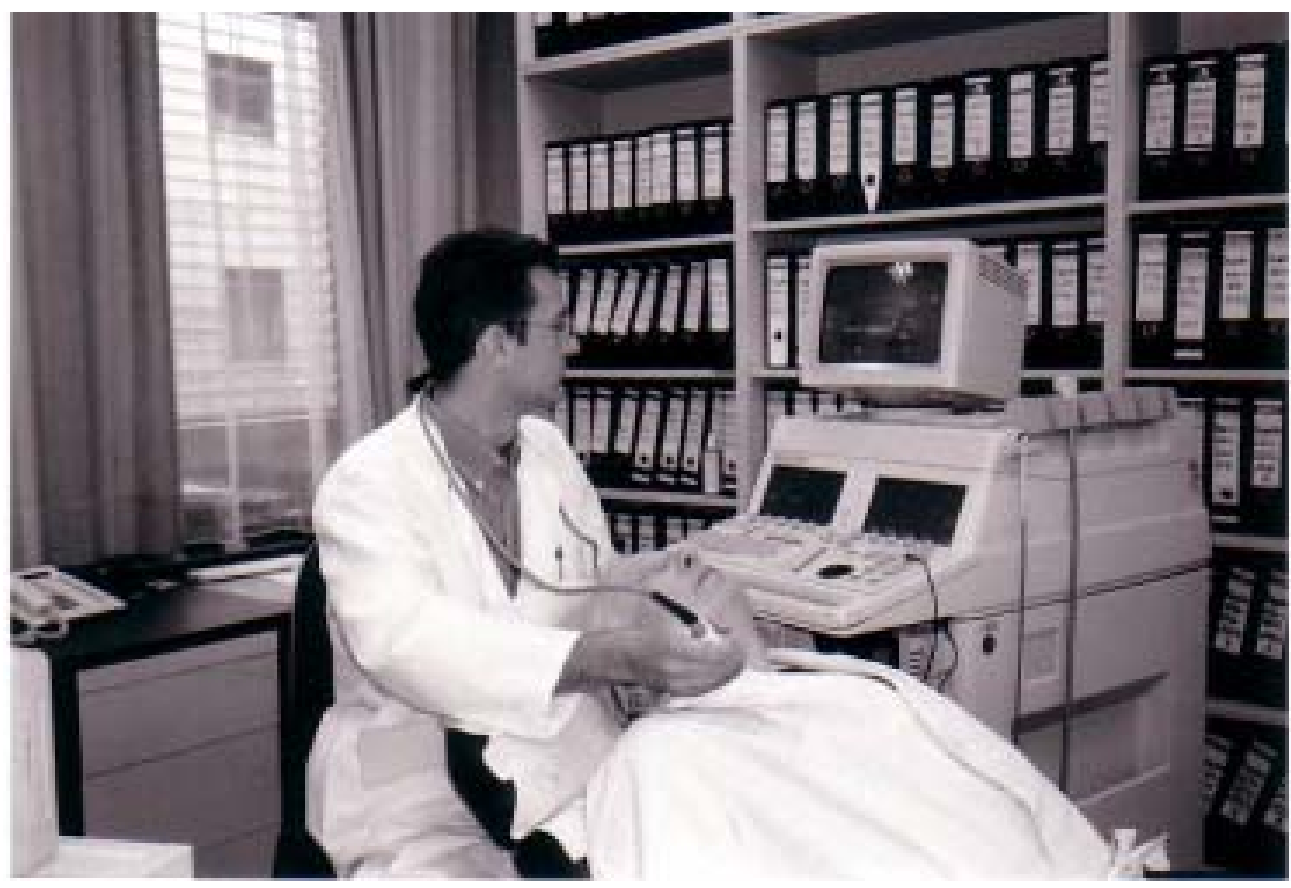




\subsection{Patienten}

An der Studie nahmen 121 Patienten und Probanden in einem Zeitraum von ca. 12 Monaten teil, davon 44 Frauen und 77 Männer im Alter von 32-88 Jahren (Mittelwert 64,5 $\pm 12,5$ ). Eine Altersbegrenzung erfolgte nicht. Ausgeschlossen wurden Patienten nach Thrombendarteriektomie $(\mathrm{n}=3)$ und Personen mit schlechter Bildqualität und dadurch unklarer Wiedergabe der charakteristischen Echostruktur $(n=10)$. Es handelt sich um konsekutive Patienten und Probanden, welche sich von August 1997 bis August 1998 in stationärer oder ambulanter Behandlung der neurologischen Abteilung im Klinikum Großhadern befanden. Die untersuchten Studienteilnehmer wurden in die folgenden vier Hauptgruppen unterteilt.

\subsubsection{Stenosen/Verschlüsse im karotidalen Stromgebiet (KS)}

Die erste Gruppe bestand aus 57 Patienten, 17 Frauen und 40 Männer im Alter von 68 (Median) Jahren, mit karotidalen Stenosen oder Verschlüssen (Tab. 2). Einige Personen dieser Gruppe zeigten zum Zeitpunkt der Untersuchung Symptome, die von TIA's bis zum kompletten Insult reichten. Auch Patienten mit koronarer Herzkrankheit (KHK) und rezidivierender Angina pectoris wurden als symptomatisch eingestuft.

Stenosen $\geq 50 \%$ wurden als hämodynamisch wirksam definiert und in diese Gruppe aufgenommen. 29 Stenosen (davon 5 Verschlüsse) der ACI waren auf der linken Seite zu finden und 30 (davon 2 Verschlüsse) auf der rechten Seite. 26 Patienten hatten eine Stenose der linken A. carotis externa (ACE) und 16 Patienten der rechten ACE. Patienten mit Stenosen der ACC wurden nicht in die Studie mit einbezogen. Stenosen kleiner 50\% wurden als hämodynamisch irrelevant bewertet. Sie wurden nicht in die Stenosegruppen eingeschlossen. Die Zuordnung zu den einzelnen Gruppen erfolgte aufgrund der Befunde der extrakraniellen Doppler- und Duplexuntersuchung und nach den üblichen dopplersonographischen Kriterien [WIDDER 95]. 


\subsubsection{Stenosen/Verschlüsse im vertebrobasilären Stromgebiet (VBS)}

16 Patienten, 6 Frauen und 10 Männer, im Alter von 72,5 (Median) Jahren mit vertebrobasilären hämodynamisch wirksamen Stenosen und Verschlüssen (Tab. 2) wurden in die zweite Gruppe eingeteilt. Einige Patienten waren zum Zeitpunkt der Untersuchung symptomatisch. Die klinische Symptomatik variierte von Schwindel über TIA bis zum Insult. 4 Patienten hatten zusätzlich kardiovaskuläre Erkrankungen wie z.B. chronisches Vorhofflimmern (VHF), KHK oder Zustand nach Myokardinfarkt.

Die Vertebralisstenose war bei 5 Patienten links lokalisiert und bei 3 Patienten rechts. 2 Patienten hatten Vertebralisstenosen auf beiden Seiten und ein Patient hatte eine kombinierte langstreckige vertebrobasiläre Stenose. 2 Verschlüsse der A. vertebralis waren auf der linken Seite zu finden und einer auf der rechten Seite. Bei 3 Patienten wurde eine Stenose der A. basilaris diagnostiziert.

\subsubsection{Stenosen/Verschlüsse im karotidalen und vertebrobasilären Stromgebiet (KS/VBS)}

Die dritte Gruppe bestand aus 16 Patienten, 4 Frauen und 12 Männer, im Alter von 74,5 (Median) Jahren mit gleichzeitig bestehenden karotidalen und vertebrobasilären Stenosen und Verschlüssen (Tab. 2).

8 hämodynamisch wirksame Makroangiopathien der A. vertebralis waren auf der linken Seite zu finden, davon 5 Stenosen und 3 Verschlüsse. Wir sahen 3 hämodynamisch wirksame Makroangiopathien auf der rechten Seite, davon 2 Stenosen und 1 Verschluß. In der linken ACI erfaßten wir 11 und in der rechten 8 Stenosen, davon 2 Verschlüsse links und einer rechts.

Wir haben die Studienteilnehmer nochmals in vier Gruppen mit unterschiedlichen Arteriosklerosestadien eingeteilt (1. ohne Stenose 2. mit einer Stenose 3. mindestens zwei Stenosen 4. Verschluß in den hirnversorgenden Arterien) und definierten dann, daß sich die Probanden der ersten Gruppe in einem frühen Stadium und die Probanden der vierten Gruppe in einem fortgeschrittenen Stadium der Arteriosklerose befanden. Schließlich haben wir die Mittelwerte der IMT zwischen den vier Gruppen verglichen (einfaktorielle ANOVA). 


\subsubsection{Kontrollgruppe}

32 Kontrollpersonen (Tab. 2), 17 Frauen und 15 Männer im Alter von 52 (Median) Jahren waren symptomlos. Keiner der Probanden litt an zerebrovaskulären Erkrankungen. Sie hatten weder Stenosen noch Verschlüsse. Diese Probanden dienten in der vierten Gruppe als Kontrollkollektiv.

\subsection{Patientenfragebogen}

Alle Studienteilnehmer mußten einen Fragebogen ausfüllen, der klassische kardiovaskuläre Risikofaktoren, vorangehende zerebrale und koronare Ereignisse, frühere Operationen und die aktuelle Medikation erfaßte. Fehlende oder mangelhafte Angaben wurden aus den Patientenakten ergänzt. Der Fragebogen wurde nach folgenden Kriterien ausgewertet.

\subsubsection{Klassische zerebrovaskuläre Risikofaktoren}

90\% der von uns untersuchten Studienteilnehmer hatten mindestens einen der zerebrovaskulären Risikofaktoren. Nur $10 \%$ der Teilnehmer gaben keinen der nachfolgenden Risikofaktoren an.

Arterielle Hypertonie wurde angenommen, wenn der Patient erhöhte Blutdruckwerte (systolisch>160 mmHg, diastolisch>95 mmHg) zeigte und/oder Antihypertonika einnahm. Mehr als die Hälfte aller Teilnehmer (52\%) waren Hypertoniker.

Hypercholesterinämie wurde definiert als ein Gesamtspiegel des Plasmacholesterins von $>200 \mathrm{mg} / \mathrm{dl}$ in den vorherigen 12 Monaten. Bei Einnahme von lipidsenkenden Medikamenten und dokumentierter Hypercholesterinämie wurde dieser Risikofaktor ebenfalls als positiv bewertet. $46 \%$ der Teilnehmer unserer Studie hatten eine Hypercholesterinämie zum Zeitpunkt der Untersuchung.

18\% unserer Patienten litten an Diabetes mellitus. Wir schlossen Patienten in diese Risikogruppe ein, falls eine positive Anamnese vorlag und/oder der Patient mit Insulin oder blutzuckersenkenden Medikamenten behandelt wurde.

Die Familienanamnese war positiv, wenn bei Verwandten ersten Grades zerebrale und/oder koronare Ereignisse zu eruieren waren. Etwa ein Drittel (34\%) der von uns untersuchten Patienten hatten eine positive Familienanamnese. 
23\% der Studienteilnehmer waren Raucher. Nur Personen, die zum Zeitpunkt der Untersuchung rauchten, wurden dieser Gruppe zugeteilt. Ehemalige Raucher wurden als Nichtraucher eingestuft.

$\mathrm{Zu}$ den vorangehenden koronaren Ereignissen zählten wir eine Anamnese von Angina pectoris, Myokardinfarkten, koronaren Angioplastien oder Bypasschirurgie. 57\% unserer Patienten litten an einer chronisch-ischämischen Herzerkrankung.

TIA's, RIND's (reversibles neurologisches Defizit) und komplette Insulte wurden als Untergliederung der zerebrale Ereignisse gewählt.

Auch Alter und Geschlecht wurden zu den klassischen zerebrovaskulären Risikofaktoren gezählt und auf dem Patientenfragebogen vermerkt.

\subsubsection{Frühere Operationen}

Die Anamnese der operativen Eigriffe gab Aufschluß über OP's an den hirnversorgenden Arterien oder am zentralen Nervensystem. Bei Zustand nach Thrombendarteriektomie der Karotiden wurde der Patient von dieser Studie ausgeschlossen. Auch andere chirurgische Eingriffe wie z.B. Tumorresektionen konnten wichtige Hinweise auf den Gesundheitszustand des Patienten geben.

\subsubsection{Aktuelle Medikation}

Alle Medikamente, die der Studienteinehmer zum Zeitpunkt der Untersuchung einnahmen, wurden notiert. Die Dosierung wurde in Milligramm angegeben. Die Verteilung der Tagesdosis auf morgens, mittags und abends wurde berücksichtigt. Nahmen die Patienten Antihypertonika, Lipidsenker oder Insulin ein, so konnten sie in die jeweilige Risikogruppe eingeteilt werden. Bei Einnahme von Kontrazeptiva (Östrogene) wurde von einem erhöhtem Risikopotential für zerebrale und koronare Ereignisse ausgegangen werden. 


\section{PATIENTENFRAGEBOGEN}

Datum: .Nummer:

Name: .Vorname:

Geburtsdatum:

\section{Risikofaktoren}

Hypercholesterinämie (mg/dl): . seit wann:

Art. Hypertonie (mmHg): . seit wann:

Nikotinabusus (Zigaretten/Tag): . seit wann:

Alkohol (ml/Tag): seit wann:

Diabetes mellitus (mg/dl): .Typ: .seit wann:

Familienanamnese:

Geschlecht: .Alter:

Größe: .Gewicht:

\section{Herz- Kreislauferkrankungen}

TIA; RIND; kompletter Hirninfarkt:

Periphere AVK:

Angina pectoris, Myokardinfarkt:

\section{Operationen}

\section{vorangehende Doppler- Untersuchung}

Datum: .Nummer:

\section{vorangehende Duplex- Untersuchung}

Datum: .Nummer:

\section{Aktuelle Medikation (Präparat, Dosis)}




\subsection{Statistische Auswertung}

Die Daten wurden in der Regel als Mittelwert \pm Standardabweichung angegeben. Die Intima-Media-Thickness (IMT) wurde seitengetrennt (IMTR"rechts" bzw. IMTL "links") und als Mittelwert $(\mathrm{IMT}=$ rechts + links/2) dargestellt. Auch der innere Querdurchmesser (QD) wurde seitengetrennt (QDR bzw. QDL) und als Mittelwert (QD = rechts + links/2) angegeben. Das Alter in den einzelnen Stenosegruppen wurde als Median angegeben. Für den Vergleich der IMT- bzw. QD-Messungen wurde der unverbundene bzw. verbundene TTest angewendet. Dabei wurde der F-Test (Levene-Test der Varianzengleichheit) berücksichtigt. Zwischen IMT und QD wurde die Korrelation nach Pearson berechnet und im klassischen Streudigramm dargestellt. Die Risikofaktoren wurden als gruppiertes Merkmal (ja/nein) dargestellt und der Mittelwertvergleich der IMT wurde ebenfalls mit dem T-Test ausgewertet. Die Studienteilnehmer wurden in vier Gruppen mit unterschiedlichen Arteriosklerosestadien eingeteilt (1. ohne Stenose 2. eine Stenose 3. mindestens zwei Stenosen 4. Verschluß). Zunächst wurde der Test der Homogenität der Varianzen durchgeführt. Der Levene-Test zeigte keine signifikante Abweichung von der Homogenität der Varianzen. Es konnte also die klassische ANOVA-Tabelle interpretiert werden. Die Mittelwerte der IMT zwischen den vier Gruppen wurden verglichen (einfaktorielle ANOVA). Zwischen Alter und IMT bzw. QD wurde die Korrelation (Spearman) sowie die Regression geprüft und das Bestimmtheitsmaß berechnet. Statistische Signifikanz wurde bei $\mathrm{p}<0,05$ angenommen. 


\section{Ergebnisse}

\subsection{Intima-Media-Thickness bei vertebrobasilärer und karotidaler Makroangiopathie}

\section{Folgende Ergebnisse wurden neu gefunden:}

\subsubsection{Intima-Media-Thickness}

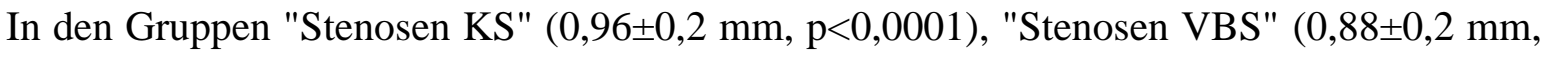
$\mathrm{p}<0,001)$ und "Stenosen KS/VBS" $(0,99 \pm 0,4 \mathrm{~mm}, \mathrm{p}<0,003)$ findet sich eine signifikant erhöhte IMT gegenüber den Kontrollpersonen $(0,67 \pm 0,1 \mathrm{~mm})$. Die IMT der Patienten mit VBS-Makroangiopathie ist tendenziell niedriger als die IMT der Patienten mit KSMakroangiopathie, ohne daß dieser Unterschied eine statistische Signifikanz erreicht (Tab. 2, Abb. 4). Bei Vergleich der Mittelwerte der IMT zwischen Patienten mit sowohl karotidalen als auch vertebrobasilären Stenosen/Verschlüssen ("Stenosen KS/VBS") und Patienten mit rein karotidalen ("Stenosen KS") bzw. rein vertebrobasilären ("Stenosen VBS") Stenosen/Verschlüssen zeigt sich kein signifikanter Unterschied (Tab. 2). 


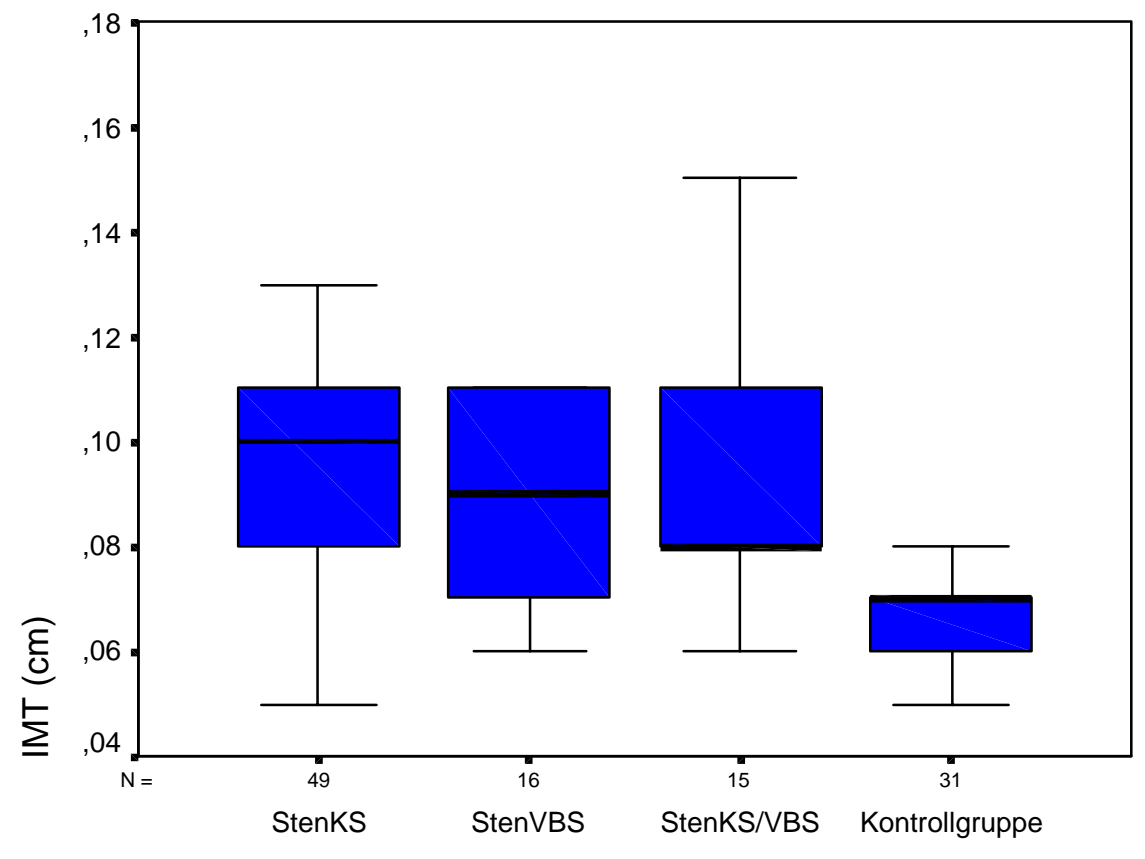

Gruppe

Abb. 4 Boxplots der Intima-Media-Thickness (IMT) in cm der A. carotis communis Vergleich zwischen den Gruppen Stenosen KS, Stenosen VBS, Stenosen KS/VBS und der Kontrollgruppe. KS = karotidales Stromgebiet, VBS = vertebrobasiläres Stromgebiet 
Tab. 2. Intima-Media-Thickness (IMT) und innerer Querdurchmesser (QD) in den Stenosegruppen im Vergleich

\begin{tabular}{|c|c|c|c|c|}
\hline & $\begin{array}{c}\text { Stenosen KS } \\
(n=57)\end{array}$ & $\begin{array}{c}\text { Stenosen VBS } \\
(\mathrm{n}=16)\end{array}$ & $\begin{array}{c}\text { Stenosen } \\
\text { KS/VBS } \\
(n=16)\end{array}$ & $\begin{array}{c}\text { Kontrolle } \\
(n=32)\end{array}$ \\
\hline Alter (Median) & 68 & 72,5 & 74,5 & 52 \\
\hline $\operatorname{Sex}(m / w)$ & $40 / 17$ & $10 / 6$ & $12 / 4$ & $15 / 17$ \\
\hline $\mathrm{IMT}_{(\mathrm{mm})}$ & $0,96 \pm 0,2 *$ & $0,88 \pm 0,2^{* *}$ & $0,99 \pm 0,4 * * *$ & $0,67 \pm 0,1$ \\
\hline $\mathbf{Q D}_{(\mathrm{mm})}$ & $6,6 \pm 1,2^{\circ}$ & $6,9 \pm 0,9^{\circ \circ}$ & $7,4 \pm 1,4^{\circ \circ \circ}$ & $5,8 \pm 1,2$ \\
\hline \multicolumn{5}{|c|}{ 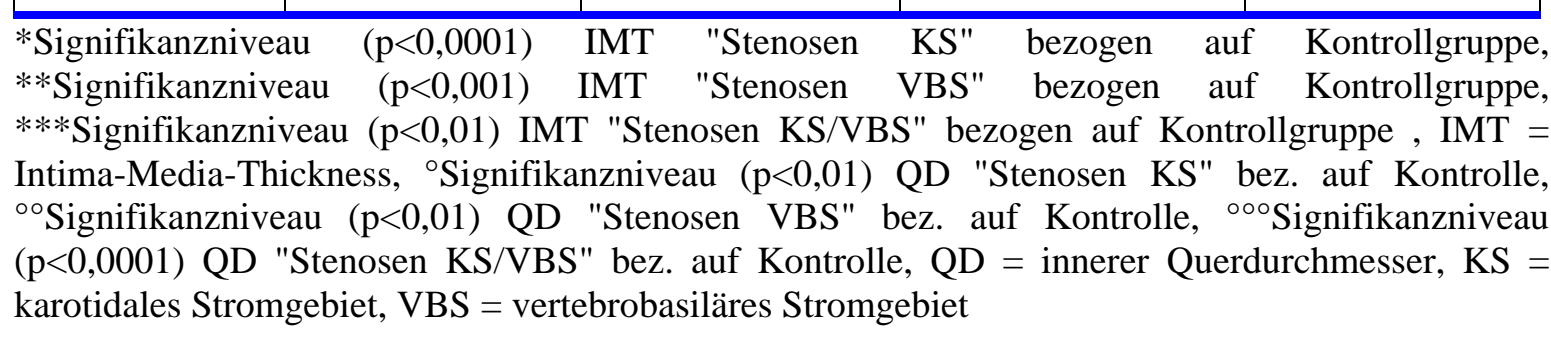 } \\
\hline
\end{tabular}




\subsubsection{Querdurchmesser}

Der QD in der Gruppe mit überwiegend karotidalen Stenosen "KS" beträgt 6,6 $\pm 1,2 \mathrm{~mm}$. In

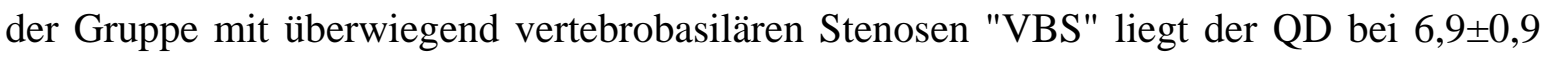
mm. In der Gruppe mit Stenosen sowohl im karotidalen als auch im vertebrobasilären Stromgebiet "KS/VBS" ergibt der QD einen Wert von 7,4 $\pm 1,4 \mathrm{~mm}$. Im Vergleich zur Kontrollgruppe mit 5,8 $\pm 1,2 \mathrm{~mm}$ ist in allen Stenosegruppen ein signifikant größerer QD der ACC zu beobachten (Tab. 2, Abb. 5). Bei Vergleich des QD der einzelnen Stenosegruppen untereinander war kein signifikanter Unterschied zu finden.

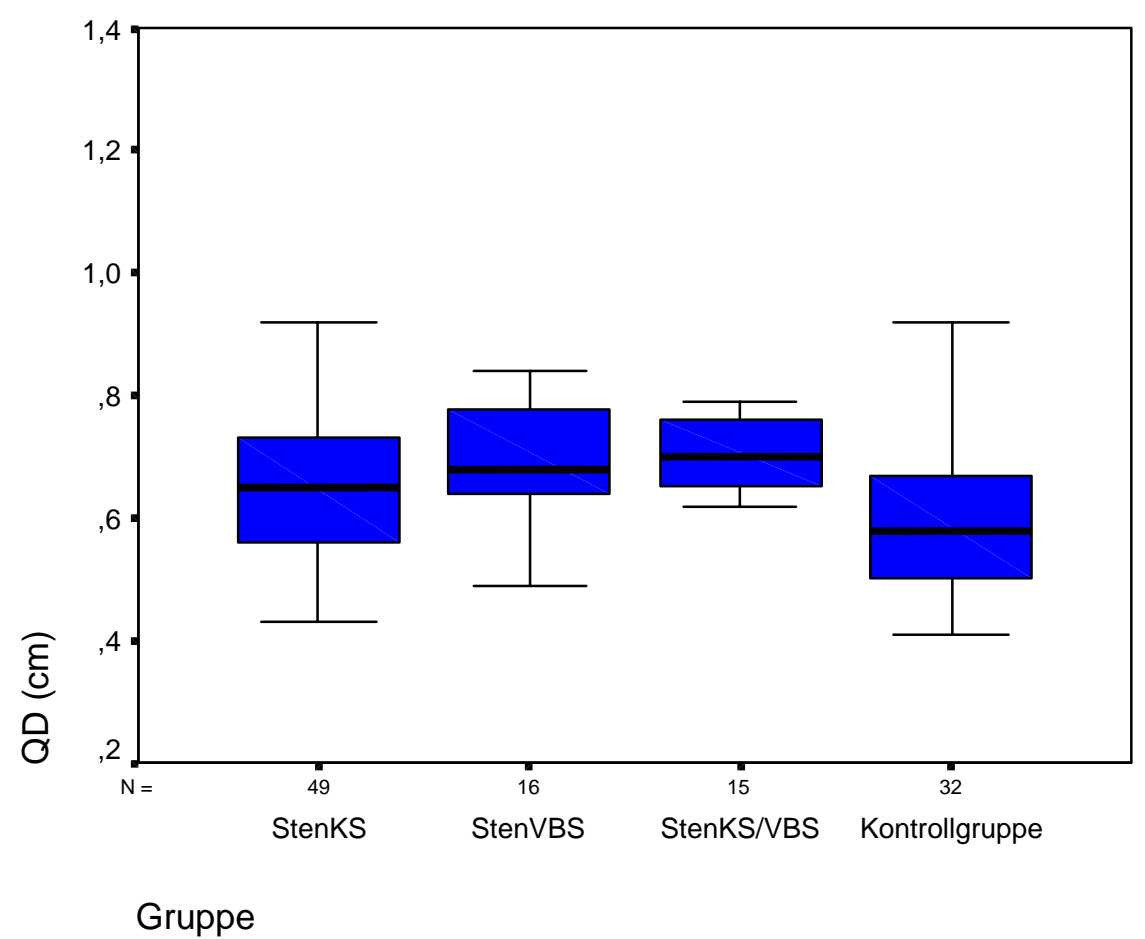

Abb. 5 Boxplots des inneren Querdurchmessers (QD) in cm der A. carotis communis Vergleich zwischen den Gruppen Stenosen KS, Stenosen VBS, Stenosen KS/VBS und der Kontrollgruppe. KS = karotidales Stromgebiet, VBS = vertebrobasiläres Stromgebiet 
Es besteht ein positiver linearer Zusammenhang zwischen dem QD und der IMT aller Studienteilnehmer (Abb. 6). Zwischen QD und IMT zeigt sich eine geringe Korrelation $(\mathrm{r}=0,46)$, welche beidseitig höchst signifikant ist $(\mathrm{p}<0,0001$, Tab. 3$)$.

Tab. 3 Korrelation nach Pearson zwischen innerem Querdurchmesser (QD) und Intima-Media-Thickness (IMT)

\section{Korrelationen}

\begin{tabular}{|ll|r|r|}
\hline & & \multicolumn{1}{|c|}{ QD } & \multicolumn{1}{|c|}{ IMD } \\
\hline QD & Korrelation nach Pearson & 1,000 &, $460 *$ \\
& Signifikanz (2-seitig) &, &, 000 \\
& $\mathrm{~N}$ & 112 & 110 \\
\hline IMT & Korrelation nach Pearson &, $460^{* *}$ & 1,000 \\
& Signifikanz (2-seitig) &, 000 &, \\
& $\mathrm{~N}$ & 110 & 111 \\
\hline
\end{tabular}

**. Die Korrelation ist auf dem Niveau von 0,01 (2-seitig) signifikant. 


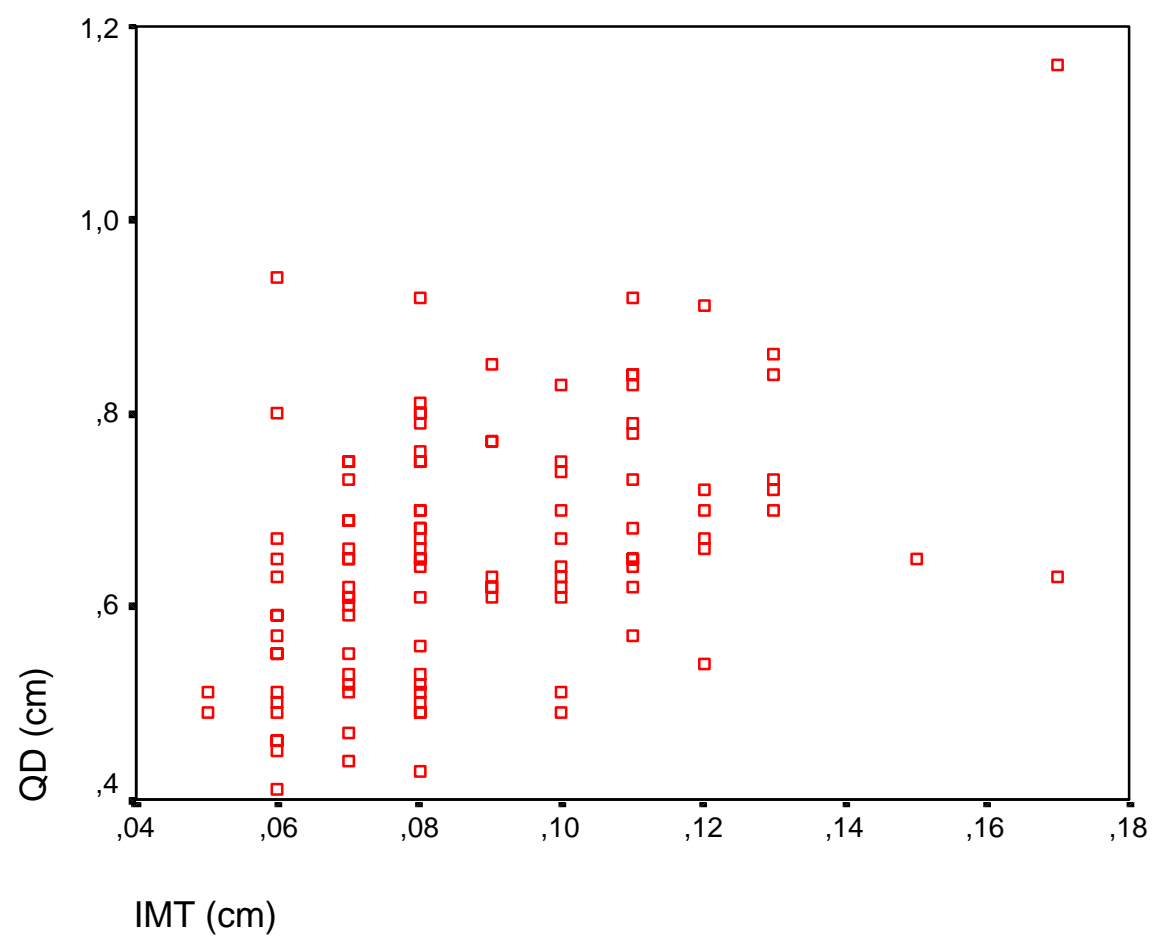

Abb. 6 Streudiagrammm - Korrelation zwischen innerem Querdurchmesser (QD) und Intima-Media-Thickness (IMT) der A. carotis communis in cm aller Studienteilnehmer 


\subsection{Graduierung der Stenosen/Verschlüsse}

Wir unterteilen alle Studienteilnehmer in vier Gruppen. In der ersten Gruppe $(n=31)$ sind Probanden ohne Stenosen/Verschlüsse in den hirnversorgenden Gefäßen. Die zweite Gruppe ( $\mathrm{n}=35$ ) besteht aus Patienten mit nur einer Stenose. Mindestens zwei Stenosen haben die Patienten in der dritten Gruppe $(n=31)$. Patienten mit Verschlüssen werden in die vierte Gruppe $(n=13)$ eingeteilt.

Nach Berechnung der Mittelwerte der IMT in den vier Gruppen, vergleichen wir die Mittelwerte untereinander (ANOVA). Die Unterschiede zwischen den Gruppen insgesamt sind höchst signifikant ( $\mathrm{p}<0,001$, Tab. 4).

Tab. 4 Vergleich der Intima-Media-Thickness (IMT) nach einfaktorieller ANOVA zwischen den vier Gruppen (1. ohne Stenose/Verschluß, 2. eine Stenose, 3. mindestens zwei Stenosen, 4. Verschluß)

ANOVA

IMT
\begin{tabular}{|l|c|r|c|c|r|}
\hline & $\begin{array}{c}\text { Quadrats } \\
\text { umme }\end{array}$ & df & $\begin{array}{c}\text { Mittel der } \\
\text { Quadrate }\end{array}$ & F & Signifikanz \\
\hline Zwischen den Gruppen & $2,093 \mathrm{E}-02$ & 3 & $6,977 \mathrm{E}-03$ & 16,908 &, 000 \\
Innerhalb der Gruppen & $4,374 \mathrm{E}-02$ & 106 & $4,127 \mathrm{E}-04$ & & \\
Gesamt & $6,468 \mathrm{E}-02$ & 109 & & & \\
\hline
\end{tabular}

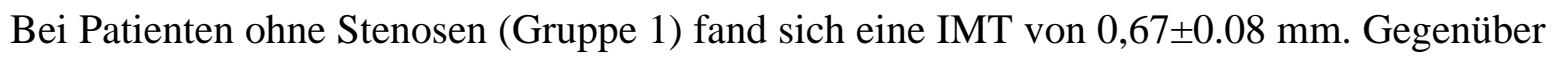
den Probanden aus der ersten Gruppe zeigten die Patienten aus der zweiten (0,91 $\pm 0,22 \mathrm{~mm}$, $\mathrm{p}<0,001)$, dritten Gruppe $(1,03 \pm 0,26 \mathrm{~mm}, \mathrm{p}<0,001)$ und vierten Gruppe $(0,95 \pm 0,21 \mathrm{~mm}$, $\mathrm{p}<0,001$ ) eine höchst signifikante Zunahme der IMT (Tab. 5, Abb. 7). 
Tab. 5 Intima-Media-Thickness (IMT) in der A. carotis communis - Vergleich zwischen den Gruppen ohne Stenosen, mit einer Stenose, mit mindestens zwei Stenosen und mit Verschluß

\begin{tabular}{|l|c|c|c|c|}
\hline & keine & 1-Gefäß & mehr-Gefäß & Verschluß \\
\hline Anzahl & $\mathrm{n}=31$ & $\mathrm{n}=35$ & $\mathrm{n}=31$ & $\mathrm{n}=13$ \\
\hline IMT (mm) & $0,67 \pm 0,08$ & $0,91 \pm 0,22^{*}$ & $1,03 \pm 0,26 *$ & $0,95 \pm 0,21 *$ \\
\hline
\end{tabular}

*Signifikanzniveau $(\mathrm{p}<0,0001)$ bezogen auf IMT bei Patienten ohne Stenosen/Verschlüsse, keine = keine Stenosen oder Verschlüsse in den hirnversorgenden Arterien (ACI, ACE, VA u. BA), 1-Gefäß = eine Stenose in den in den hirnversorgenden Arterien, mehr-Gefäß = mindestens zwei Stenosen in den hirnversorgenden Arterien, $\mathrm{ACI}=\mathrm{A}$. carotis interna, $\mathrm{ACE}=\mathrm{A}$. carotis externa, $\mathrm{VA}=\mathrm{A}$. vertebralis, BA $=$ A. basilaris, IMT = Intima-Media-Thickness (Mittelwert \pm Standardabweichung)

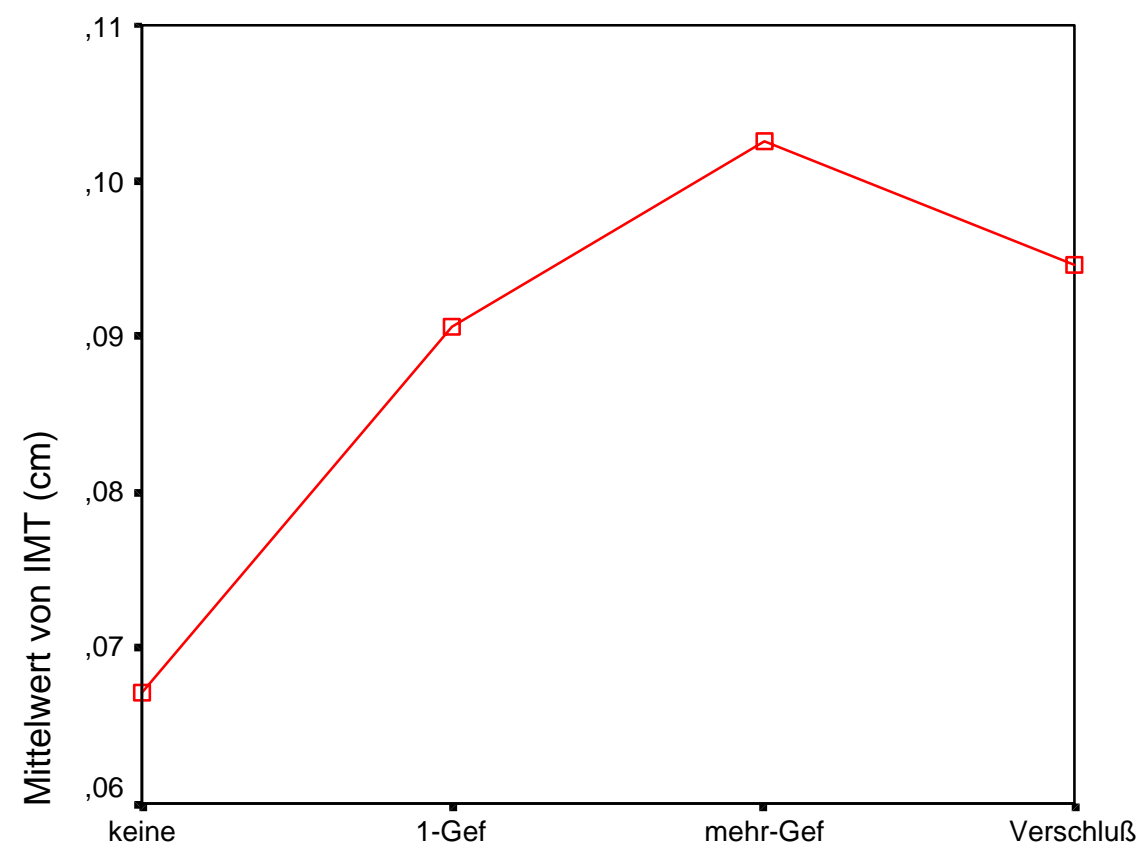

Gefstat

Abb. 7 Intima-Media-Thickness (IMT) in cm der A. carotis communis nach Graduierung der Stenosen/Verschlüsse, Gefstat = Gefäßstatus: keine Stenosen, 1-Gef = eine Stenose, mehr-Gef = > eine Stenose 


\subsection{Messung der IMT der A. carotis communis (ACC)}

\section{Folgende Ergebnisse wurden bestätigt:}

\subsubsection{Männer und Frauen}

Die IMT in der ACC (s. Kap. 3.2.4.) von allen Patienten und Kontrollpersonen im mittleren Alter von $64,5 \pm 12,5$ Jahren liegt bei $0,88 \pm 0,2 \mathrm{~mm}$. Mit 0,92 $\pm 0,2 \mathrm{~mm}$ ist die IMT bei Männern signifikant $(\mathrm{p}<0,05)$ dicker als bei Frauen $(0,81 \pm 0,2 \mathrm{~mm})$.

\subsubsection{Rechte und linke ACC}

Die IMT zeigt auf der linken Seite tendenziell höhere Werte als auf der rechten Seite. Der Vergleich der Seitendifferenz der IMT ergibt jedoch keine Signifikanz. Auch bei getrennter Betrachtung von Männern und Frauen ist die IMT auf der linken und rechten Seite annähernd gleich und zeigt keine signifikanten Unterschiede (Tab. 6, Abb. 8).

Tab. 6 Intima-Media-Thickness (IMT) in der A. carotis communis - Vergleich zwischen Männern und Frauen sowie zwischen der rechten und linken Seite

\begin{tabular}{|l|c|c|c|}
\hline & Frauen & Männer & Insgesamt \\
& $(\mathbf{n}=44)$ & $(\mathbf{n}=77)$ & $(\mathbf{n}=121)$ \\
\hline Alter (Median) & $27-87(60)$ & $36-88(66)$ & $27-88(64)$ \\
\hline IMT (mm) & $0,81 \pm 0,2$ & $0,92 \pm 0,2^{*}$ & $0,88 \pm 0,2$ \\
\hline IMTR (mm) & $0,80 \pm 0,3$ & $0,91 \pm 0,3$ & $0,87 \pm 0,3$ \\
\hline IMTL (mm) & $0,81 \pm 0,2$ & $0,93 \pm 0,3$ & $0,88 \pm 0,3$ \\
\hline
\end{tabular}

* Signifikanzniveau $(\mathrm{p}<0,05)$ bezogen auf IMT Frauen, IMT = Intima-Media-Thickness (Mittelwert \pm Standardabweichung), IMTR = IMT der rechten A.carotis communis, IMTL = IMT der linken A. carotis communis 


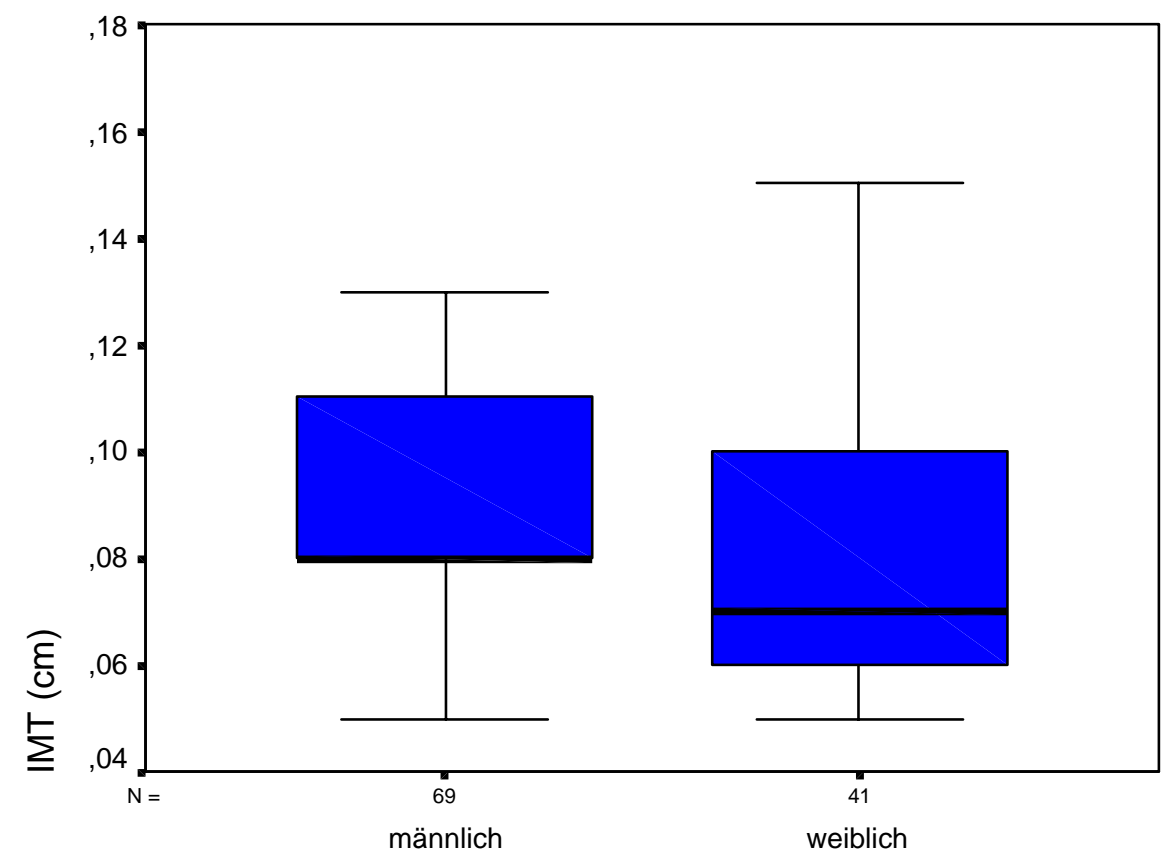

Geschlecht

Abb. 8 Boxplots der Intima-Media-Thickness (IMT) in cm der A. carotis communis Vergleich zwischen Männern und Frauen 


\subsection{Messung des inneren Querdurchmessers (QD) der ACC}

\subsubsection{Männer und Frauen}

Der durchschnittliche QD von allen Teilnehmern unserer Studie ergibt 6,6 $\pm 1,2 \mathrm{~mm}$. Der Unterschied des QD zwischen Frauen und Männern ist höchst signifikant $(\mathrm{p}<0,001)$ verschieden. Männer zeigen einen QD von 7,0 $\pm 1,3 \mathrm{~mm}$, während Frauen nur einen QD von $5,8 \pm 1,0 \mathrm{~mm}$ aufweisen.

\subsubsection{Rechte und linke ACC}

Der Seitenunterschied mit einem QDR (rechts) von 6,6 $\pm 1,4 \mathrm{~mm}$ und einem QDL auf der linken Seite von 6,4 $\pm 1,3 \mathrm{~mm}$ ist höchst signifikant $(\mathrm{p}<0,001)$, allerdings nur wenn man die Männergruppe testet (Tab. 7, Abb. 9) Bei Frauen zeigt sich kein signifikanter Seitenunterschied des QD.

Tab. 7 Innerer Querdurchmesser (QD) in der A. carotis communis - Vergleich zwischen Männern und Frauen sowie zwischen der rechten und linken Seite

\begin{tabular}{|l|c|c|c|}
\hline & Frauen & Männer & Insgesamt \\
& $(\mathrm{n}=44)$ & $(\mathrm{n}=77)$ & $(\mathrm{n}=121)$ \\
\hline Alter (Median) & $27-87(60)$ & $36-88(66)$ & $27-88(64)$ \\
\hline QD (mm) & $5,8 \pm 1,0$ & $6,9 \pm 1,3^{*}$ & $6,6 \pm 1,2$ \\
\hline QDR (mm) & $5,9 \pm 1,2$ & $7,1 \pm 1,4^{* *}$ & $6,6 \pm 1,4$ \\
\hline QDL (mm) & $5,8 \pm 1,0$ & $6,8 \pm 1,3$ & $6,5 \pm 1,3$ \\
\hline
\end{tabular}

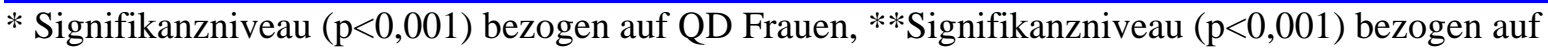
QDL bei Männern, QD = innerer Querdurchmesser (Mittelwert \pm Standardabweichung), QDR = QD der rechten A.carotis communis, QDL = QD der linken A. carotis communis 


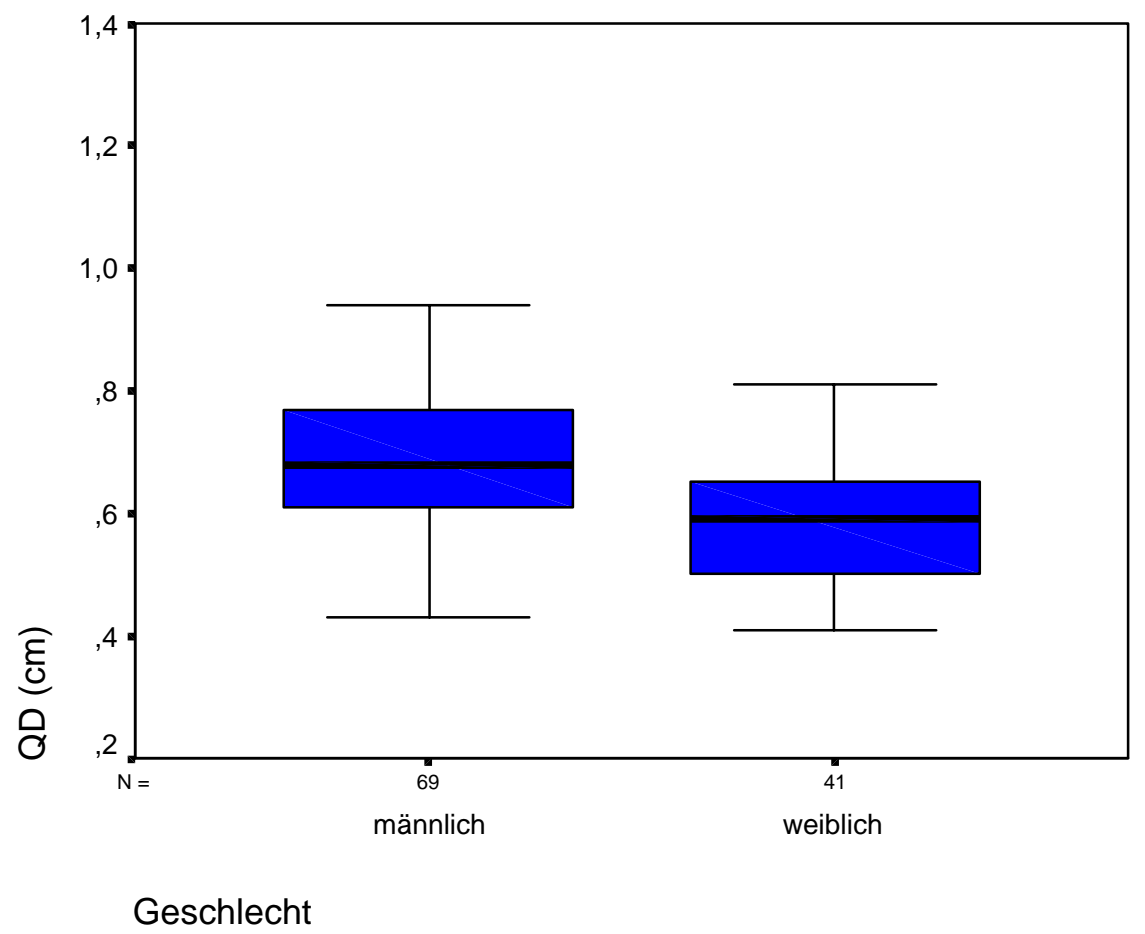

Abb. 9 Boxplots des inneren Querdurchmessers (QD) in cm der A. carotis communis Vergleich zwischen Männern und Frauen 


\subsection{Seitenverteilung der Stenosen/Verschlüsse}

Bei den Patienten aus den Stenosegruppen $(n=82)$ finden sich 184 hämodynamisch wirksame makroangiopathische Veränderungen (Stenosen/Verschlüsse). Im Schnitt zeigt jeder Patient mindestens zwei Stenosen oder Verschlüsse. Davon sind 142 mittelgradige Stenosen $(\geq 50 \%<70 \%), 24$ hochgradige Stenosen $(\geq 70 \%)$ und 18 Verschlüsse (nach dopplersonographischen Kriterien). Makroangiopathien im karotidalen Stromgebiet ( $n=152$ ) sind häufiger nachweisbar als im vertebrobasilären Stromgebiet $(n=32)$.

Von 142 mittelgradigen Stenosen sind 53 rechts und 83 links lokalisiert. Sechs Stenosen finden sich in der A. basilaris (BA). Auch in den einzelnen Gruppen (Stenosen KS, Stenosen VBS, Stenosen KS/VBS) wird eine Seitenbevorzugung links beobachtet. Bei den Patienten der Gruppe "Stenosen KS" zeigen sich z.B. 49 Makroangiopathien auf der rechten und 72 auf der linken Seite. Insgesamt sind deutlich mehr Makroangiopathien auf der linken Seite der hirnversorgenden Arterien nachzuweisen (Tab. 8). 
Tab. 8 Seitenverteilung der Stenosen und Verschlüsse in den hirnversorgenden Arterien

\begin{tabular}{|l|c|c|c|c|}
\hline & Stenosen & Stenosen & Stenosen & Insgesamt \\
& KS & VBS & KS/VBS & \\
\hline Zahl der Stenosen $<70 \%$ & 97 & 14 & 31 & 142 \\
\hline Seite (rechts/links) & $40 / 57$ & $4 / 7+3$ BA & $9 / 19+3$ BA & $53 / 83+6$ BA \\
\hline Zahl der Stenosen $\geq 70 \%$ & 17 & keine & 7 & 24 \\
\hline Seite (rechts/links) & $6 / 11$ & keine & $1 / 5+1$ BA & $7 / 16+1 B A$ \\
\hline Verschlüsse & 7 & 4 & 7 & 18 \\
\hline Seite (rechts/links) & $3 / 4$ & $1 / 2+1$ BA & $2 / 5$ & $6 / 11+1$ BA \\
\hline
\end{tabular}

Die Anzahl der Stenosen/Verschlüsse im KS ergibt sich aus der Summe aller Stenosen/Verschlüsse der ACI und ACE auf beiden Seiten. Die Zahl der Stenosen/Verschlüsse im VBS ist die Summe aller Stenosen/Verschlüsse der VA und der BA, KS $=$ karotidales Stromgebiet, $\mathrm{VBS}=$ vertebrobasiläres Stromgebiet, $\mathrm{ACI}=\mathrm{A}$. carotis interna, $\mathrm{ACE}=\mathrm{A}$. carotis externa, $\mathrm{BA}=\mathrm{A}$. basilaris, $\mathrm{VA}=\mathrm{A}$. vertebralis 


\subsection{Zerebrovaskuläre Risikofaktoren}

Hypertoniker und Diabetiker haben im Vergleich zu Nicht-Hypertonikern (Abb. 10) und Nicht-Diabetikern (Abb. 11) eine signifikant dickere IM-Schicht der ACC. Bei Rauchern sehen wir eine signifikant dünnere IM-Schicht im Vergleich zu Nicht-Rauchern (Abb. 12). Alle anderen Risikofaktoren sind zwar mit einer Erhöhung der IMT verbunden. Bei einem Vergleich der Mittelwerte zeigen diese Unterschiede aber keine Signifikanz (Tab. 9).

Patienten mit arterieller Hypertonie $(0,95 \pm 0,3 \mathrm{~mm})$ haben im Vergleich zu Studienteilnehemern ohne Bluthochdruck $(0,84 \pm 0.2 \mathrm{~mm})$ eine signifikant $(\mathrm{p}<0,05)$ erhöhte IMT. Die Hypertoniker (69 Jahre) sind im Schnitt acht Jahre älter als die Nicht-Hypertoniker (61 Jahre) (Tab. 9). Der Intima-Media-Komplex (IMT) der Diabetiker ist deutlich dicker als der der Personen ohne Diabetes mellitus $(1,03 \pm 0,2$ vs. 0,86 $\pm 0,4 \mathrm{~mm}, \mathrm{p}<0,01$, Tab. 9). Bei Rauchern findet sich mit $0,81 \pm 0,2 \mathrm{~mm}$ eine signifikant $(\mathrm{p}<0,05)$ niedrigere IMT als bei Nicht-Rauchern $(0,92 \pm 0,2 \mathrm{~mm})$. Studienteilnehmer, die rauchen (Mittelwert 57 Jahre) sind durchschnittlich zehn Jahre jünger als Teilnehmer die nicht rauchen (Mittelwert 67 Jahre). 
Tab. 9 Intima-Media-Thickness (IMT) bei Personen mit und ohne zerebrovaskulären Risikofaktor im Vergleich

\begin{tabular}{|c|c|c|c|}
\hline Risikofaktoren & ja & nein & Sig (2-seitig) \\
\hline $\begin{array}{l}\text { Arterieller Hypertonus } \\
\text { (IMT) } \\
\text { Alter (Median) }\end{array}$ & $\begin{array}{c}0,95 \pm 0,3 \mathrm{~mm} \\
32-88(69)\end{array}$ & $\begin{array}{c}0,84 \pm 0,2 \mathrm{~mm} \\
28-85(61)\end{array}$ & $\bar{~} \overline{p<<0,01}$ \\
\hline $\begin{array}{l}\text { Hypercholesterinämie (IMT) } \\
\text { Alter (Median) }\end{array}$ & $\begin{array}{c}0,93 \pm 0,2 \mathrm{~mm} \\
28-87(65)\end{array}$ & $\begin{array}{c}0,85 \pm 0,2 \mathrm{~mm} \\
32-88(64)\end{array}$ & $p<0,098$ \\
\hline $\begin{array}{l}\text { Diabetes mellitus (IMT) } \\
\text { Alter (Median) }\end{array}$ & $\begin{array}{c}1,03 \pm 0,2 \mathrm{~mm} \\
45-85(69)\end{array}$ & $\begin{array}{c}0,86 \pm 0,4 \mathrm{~mm} \\
28-88(64)\end{array}$ & $p<0,01$ \\
\hline $\begin{array}{l}\text { Familienanamnese (IMT) } \\
\text { Alter (Median) }\end{array}$ & $\begin{array}{c}0,91 \pm 0,2 \mathrm{~mm} \\
28-78(63)\end{array}$ & $\begin{array}{c}0,88 \pm 0,2 \mathrm{~mm} \\
32-88(66)\end{array}$ & $p<0,588$ \\
\hline $\begin{array}{l}\text { Nikotinkonsum (IMT) } \\
\text { Alter (Median) }\end{array}$ & $\begin{array}{c}0,81 \pm 0,2 \mathrm{~mm} \\
32-82(57)\end{array}$ & $\begin{array}{c}0,92 \pm 0,2 \mathrm{~mm} \\
28-88(67)\end{array}$ & $p<0,01$ \\
\hline $\begin{array}{l}\text { Koronare Ereignisse (IMT) } \\
\text { Alter (Median) }\end{array}$ & $\begin{array}{c}0,91 \pm 0,2 \mathrm{~mm} \\
37-88(66)\end{array}$ & $\begin{array}{r}0,86 \pm 0,3 \mathrm{~mm} \\
28-85(63)\end{array}$ & $p<0,251$ \\
\hline $\begin{array}{l}\text { Zerebrale Ereignisse (IMT) } \\
\text { Alter (Median) }\end{array}$ & $\begin{array}{r}0,93 \pm 0,2 \mathrm{~mm} \\
41-80(65)\end{array}$ & $\begin{array}{c}0,87 \pm 0,2 \mathrm{~mm} \\
28-88(65)\end{array}$ & $\mathrm{p}<0,424$ \\
\hline
\end{tabular}

Sig = Signigikanzniveau, IMT = Intima-Media-Thickness (Mittelwert \pm Standardabweichung) 


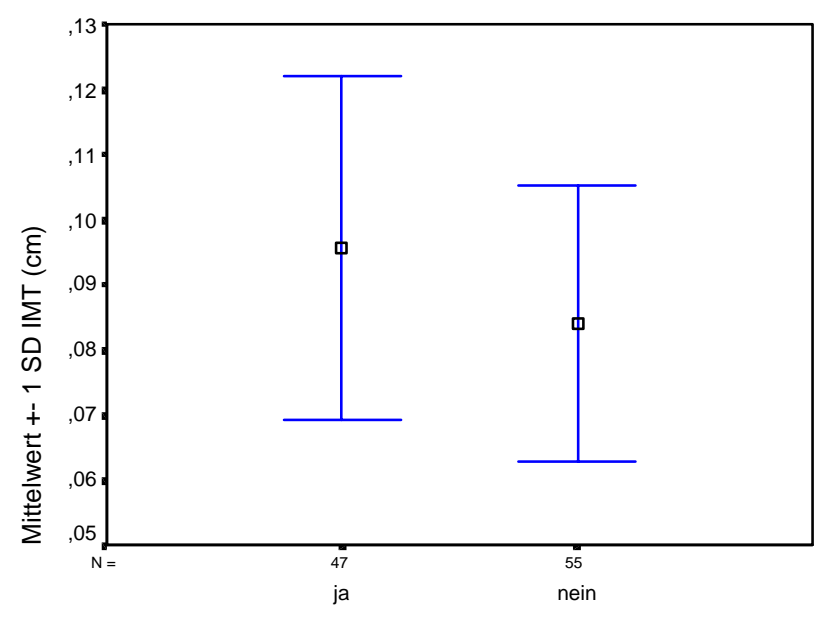

Abb. 10 IMT bei Hypertonikern und Nicht-Hypertonikern $(\mathrm{SD}=$ Standardabweichung)

Arterieller Hypertonus

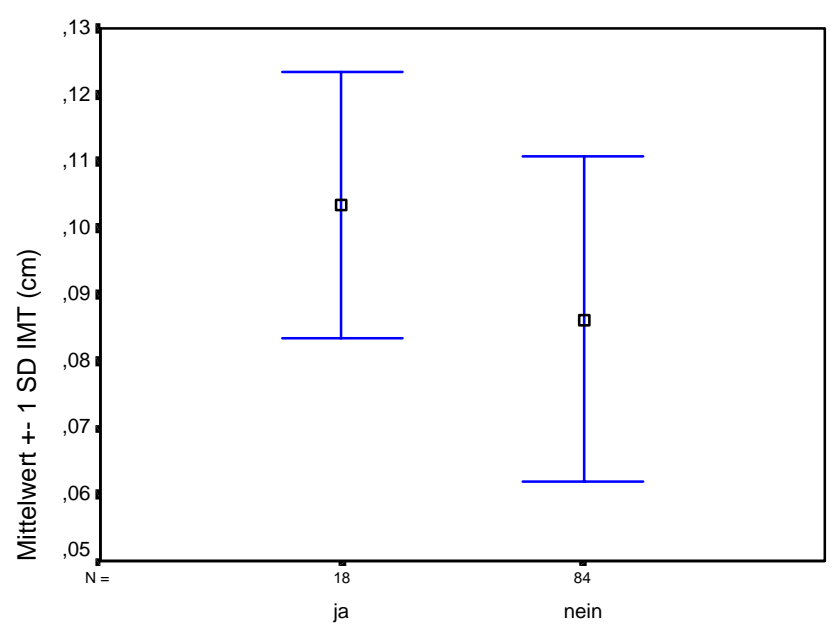

Abb. 11 IMT bei Diabetikern und NichtDiabetikern $(\mathrm{SD}=$ Standardabweichung)

Diabetes mellitus

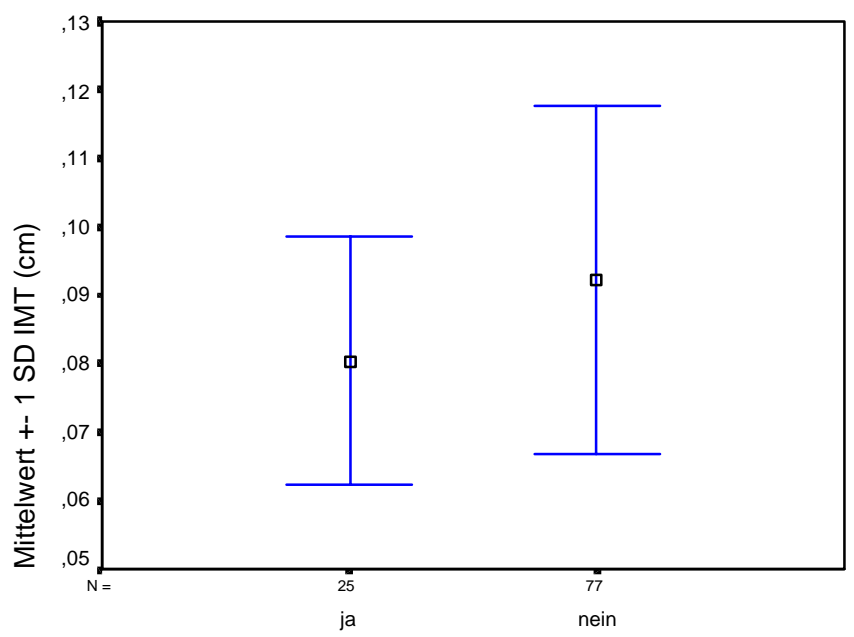

Abb. 12 IMT bei Rauchern und NichtRauchern (SD = Standardabweichung)

Nikotinabusus 
Es besteht eine positive lineare Beziehung zwischen dem Alter aller Studienteilnehmer und der IMT bzw. QD. Zwischen Alter und IMT ergibt sich eine mittlere Korrelation $(r=0,54)$, die beidseitig höchst signifikant $(p<0,0001)$ ist (Tab 10, Abb. 13). Eine geringe Korrelation $(\mathrm{r}=0,32, \mathrm{p}<0,0001)$ zeigt sich zwischen Alter und QD (Tab. 11, Abb. 14)

Tab. 10 Korrelation nach Spearman zwischen Alter und Intima-Media-Thickness (IMT) der A.carotis communis

Korrelationen

\begin{tabular}{|lll|r|r|}
\hline & & \multicolumn{1}{|c|}{ Alter } & \multicolumn{1}{|c|}{ IMT } \\
\hline Spearman-Rho & Alter & Korrelationskoeffizient & 1,000 &, $535^{\star *}$ \\
& & Sig. (2-seitig) &, 000 \\
& & 121 & 111 \\
\cline { 2 - 5 } & $\mathrm{N}$ &, $535^{\star *}$ & 1,000 \\
& IMT & Korrelationskoeffizient &, 000 & 111 \\
& Sig. (2-seitig) & 111 & 11 \\
& $\mathrm{~N}$ & & \\
& & &
\end{tabular}

**. Korrelation ist auf dem Niveau von 0,01 signifikant (2-seitig), Sig = Signifikanzniveau

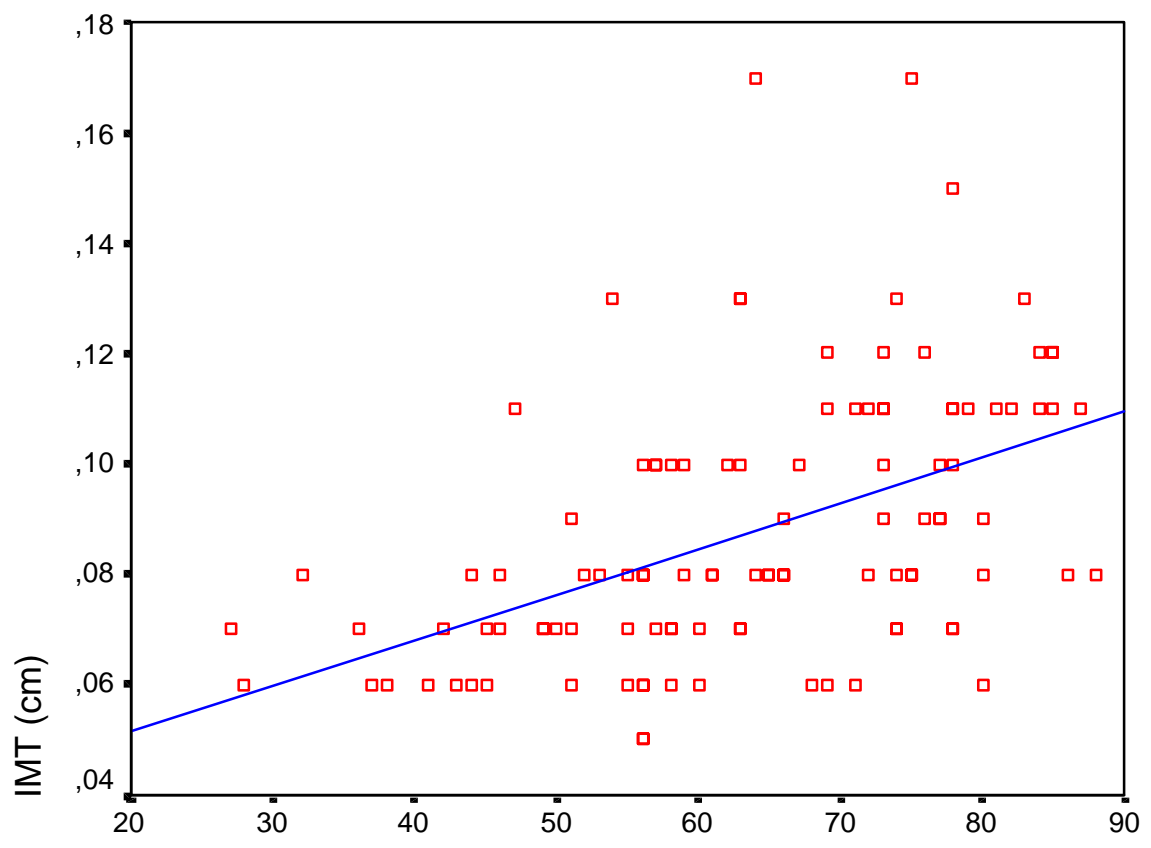

Alter 
Abb. 13 Streudiagramm mit Regressionsgerade - Korrelation und Regression zwischen Alter und Intima-Media-Thickness (IMT) der A.carotis communis

Tab. 11 Korrelation nach Spearman zwischen Alter und innerem Querdurchmesser (QD) der A. carotis communis

\begin{tabular}{|c|c|c|c|c|}
\hline \multicolumn{5}{|c|}{ Korrelationen } \\
\hline & & & QD & Alter \\
\hline \multirow[t]{6}{*}{ Spearman-Rho } & QD & Korrelationskoeffizient & 1,000 & ,321*x \\
\hline & & Sig. (2-seitig) & & ,001 \\
\hline & & $\mathrm{N}$ & 112 & 112 \\
\hline & Alter & Korrelationskoeffizient & ,321*x & 1,000 \\
\hline & & Sig. (2-seitig) & ,001 & \\
\hline & & $\mathrm{N}$ & 112 & 121 \\
\hline
\end{tabular}

${ }^{\star *}$. Korrelation ist auf dem Niveau von 0,01 signifikant (2-seitig), Sig = Signifikanzniveau 


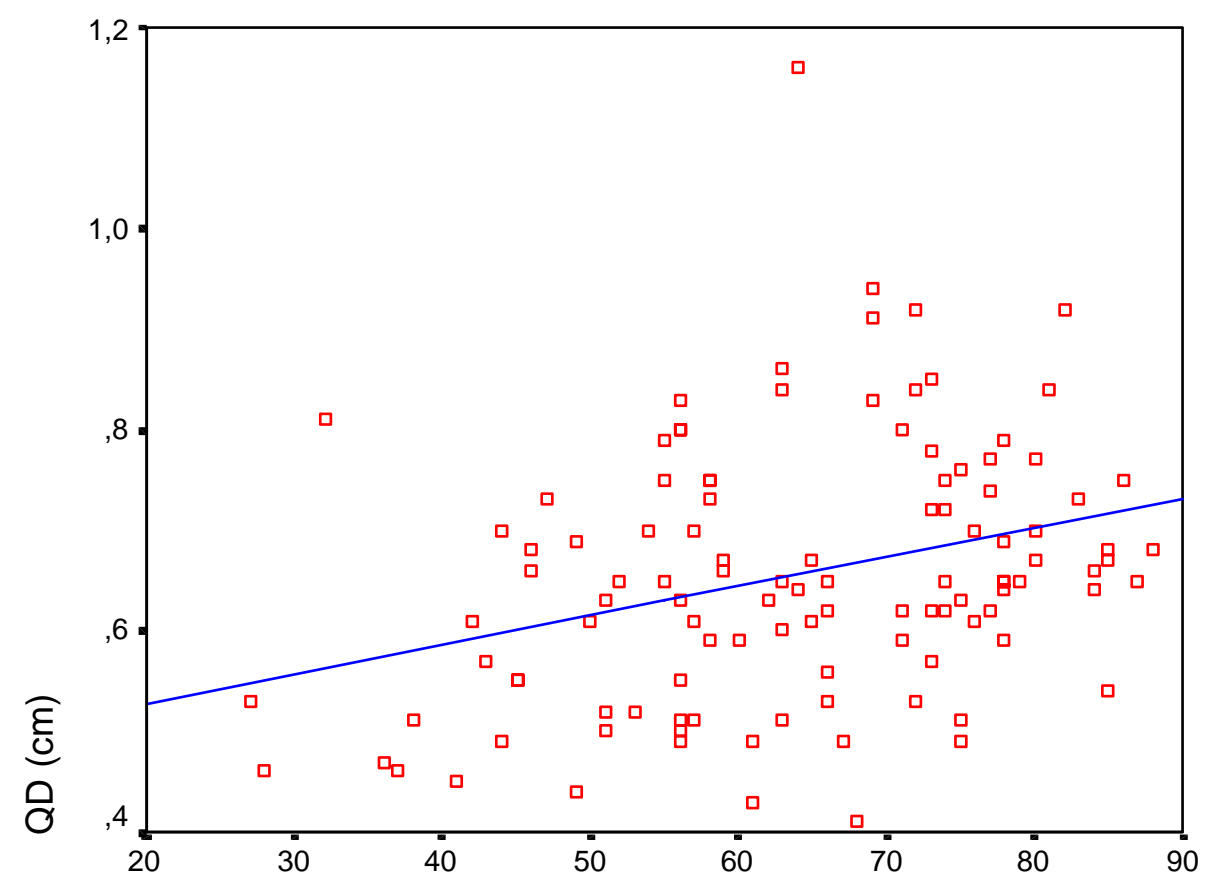

Alter

Abb. 14 Streudiagramm mit Regressionsgerade - Korrelation und Regression zwischen Alter und innerem Querdurchmesser (QD) der A.carotis communis 


\section{Diskussion}

\subsection{Intima-Media-Thickness der A. carotis communis bei vertebrobasilärer und karotidaler Makroangiopathie (Dominanztypen)}

\section{Folgendes wurde neu gefunden:}

Unsere Ergebnisse zeigten eine positive und signifikante Korrelation zwischen der IMT in der ACC und hämodynamisch wirksamen Stenosen oder Verschlüssen in den hirnversorgenden Arterien im Vergleich zu den Kontrollpersonen. Obwohl die IMT bei Patienten mit ausschließlich vertebrobasilären Makroangiopathien tendenziell geringer war als bei Patienten mit ausschließlich karotidalen Makroangiopathien, konnte dieser Unterschied keine Signifikanz erreichen (Tab. 2). Patienten mit vertebrobasilären Stenosen oder Verschlüssen waren jedoch seltener als Patienten mit karotidalen Stenosen oder Verschlüssen. Möglicherweise ist die geringere Fallzahl der Studienteilnehmer mit ausschließlich vertebrobasilären Makroangiopathien ein Grund für die fehlende Signifikanz. Trotzdem müssen wir nach unseren Ergebnissen davon ausgehen, daß die IMT bei Patienten aus beiden Gruppen vergleichbar ist.

Bei Betrachtung der karotidalen Arterien fiel eine bevorzugte Manifestation der Arteriosklerose (IMT) im Bereich der Karotisbifurkation auf [ZARINS 83]. So zeigten sich z.B. Makroangiopathien in der A. carotis interna am häufigsten an der lateralen Wand (gegenüber der Flußgabel) in den proximalen Abschnitten [FOX 82].

Dies ist wahrscheinlich auf unterschiedliche hämodynamische Bedingungen im Vergleich zur distalen A. carotis interna und proximalen A. carotis communis zurückzuführen. Die Karotisbifurkation ist eine Region der Flußteilung mit niedriger Flußgeschwindigkeit, wo der Blutfluß von der laminaren Strömung abweicht [Glagov 88]. Der Flußteiler der Karotis ist eine Prädilektiosstelle für die Entstehung von Arteriosklerose.

Es zeigte sich eine signifikante Korrelation zwischen Anzahl bzw. Größe der Makroangiopathien im karotidalen Stromgebiet und der IMT der ACC [ROSFORS 98, PERSSON 94, BONITHON-KOPP 96]. 
Unklar blieb jedoch bisher, ob sich die IMT bei Patienten mit Makroangiopathien im vertebrobasilären Stromgebiet im Vergleich zu Patienten mit Makroangiopathien im karotidalen Stromgebiet unterscheidet. Untersucht wurde hierzu die IMT bei Patienten mit hämodynamisch wirksamen makroangiopathischen Veränderungen (Stenosen/Verschlüsse) des vertebrobasilären (VBS) und des karotidalen (KS) Stromgebietes.

Nach unseren Ergebnissen müssen wir davon ausgehen, daß sich die IMT bei Patienten aus beiden Gruppen (VBS und KS) im untersuchten Patientengut nicht signifikant unterscheidet. Gesunde Probanden zeigen eine geringere IMT als Patienten mit KS-Makroangiopathie und Patienten mit VBS-Makroangiopathie.

\subsection{Graduierung der Stenosen und Verschlüsse}

Mit der Messung der IMT in den Karotiden könne die Entstehung von karotidalen Plaques vorausgesagt werden. ZUREIK et al. (2000) zeigten in einer Longitudinalstudie, daß mit zunehmender IMT auch die Wahrscheinlichkeit der Plaquebildung zunimmt und erkannten, daß die IMT ein verläßlicher Parameter zur Vorhersage von Makroangiopathien in den Karotiden sei. Auch in anderen vorangehenden Untersuchungen wurde der Verlauf der Arteriosklerose durch follow-up-Studien deutlicher als in Querschnittstudien dargestellt [BONITHON-KOPP 93, HENNERICI 85].

In unserer Studie wurde die IMT nur zu einem Zeitpunkt gemessen. Deshalb stehen keine "follow-up"-Daten zur Verfügung. Um mit unseren Ergebnissen dennoch eine Aussage über die Dynamik der Arteriosklerose gewinnen zu können, wurden die Studienteilnehmer zunächst in vier Gruppen unterschiedlicher Arteriosklerosestadien eingeteilt: 1. ohne Stenose 2. mit einer Stenose 3. mindestens zwei Stenosen 4. Verschluß in den hirnversorgenden Arterien. Dann wurde definiert, daß sich die Probanden der ersten Gruppe in einem frühen Stadium und die Patienten der vierten Gruppe in einem fortgeschrittenen Stadium der Arteriosklerose befanden. Schließlich wurden die Mittelwerte der IMT zwischen der vier Gruppen verglichen (einfaktorielle ANOVA).

Der Unterschied der IMT zwischen den vier Gruppen war höchst signifikant (Tab. 4). Beim Vergleich der einzelnen Gruppen fiel auf, daß die Gruppen mit Stenosen/Verschlüssen (Gruppe 2,3 und 4) nur jeweils im Vergleich zur Kontrollgruppe (Gruppe 1) eine 
signifikant erhöhte IMT zeigten (Tab. 5). Wenn man die Stenosegruppen einzeln miteinander verglich, ergab sich kein signifikanter Unterschied. Das heißt, Patienten mit Makroangiopathien in den hirnversorgenden Arterien zeigten eine dickere IM-Schicht als Patienten ohne Makroangiopathien. Bei Patienten mit nur einer Stenose sahen wir jedoch keine signifikant unterschiedliche IMT im Vergleich zu Patienten mit mehr als einer Stenose.

In einer Longitudinalstudie untersuchten ZUREIK et al. (2000) die Studienteilnehmer zwei und vier Jahre nach der Erstuntersuchung und sahen bei den Patienten mit der dicksten IM die deutlichste Plaquezunahme.

PERSSON et al. (1994) beschrieben den Zusammenhang zwischen der Anzahl der Plaques in den Karotiden und der IMT. Sie entwickelten einen "Plaque Score", vergleichbar mit unserer Rangskala für Stenosen, und fanden mit zunehmender Zahl der Plaques in den Karotiden einen signifikanten Anstieg der IMT.

ROSFORS et al. (1998) unterteilten Studienteilnehmer in Probanden ohne Plaques, Patienten mit Plaques und Patienten mit hämodynamisch wirksamen Stenosen in der Karotisbifurkation. Patienten mit kleineren Plaques hatten eine dickere IM als Patienten ohne Plaques, und eine dünnere IM als Patienten mit Stenosen. Analog zu unseren und den oben genannten Ergebnissen beobachteten sie eine Zunahme der IMT bei voranschreitender Arteriosklerose (Stenosen/Plaques).

BONITHON-KOPP et al. (1993) entwarfen eine Studie mit einem follow-up nach zwei Jahren. Sie beobachteten die Dynamik der Makroangiopathien (Zunahme oder Abnahme der Plaques) in den Karotiden bei bekannten kardiovaskulären Risikofaktoren und sahen eine signifikante Zunahme der Plaques bei Frauen mit IM-Verdickung im Vergleich zu Frauen mit normaler IMT.

In unserer Querschnittstudie fehlen Verlaufskontrollen, wodurch nur eine eingeschränkte Aussage über die Zunahme der IMT möglich war. Dennoch konnten wir mit unserer Rangskala für Stenosen beim Vergleich mehrerer Mittelwerte der IMT einen signifikanten Unterschied feststellen. Patienten mit Makroangiopathien hatten im Vergleich zu Probanden ohne Makroangiopathien eine dickere IM-Schicht. 
Zusammenfassung: Patienten mit Makroangiopathien in den hirnversorgenden Arterien zeigen eine erhöhte IMT im Vergleich zu Probanden ohne Makroangiopathien. Bei Patienten mit nur einer Stenose/Verschluß ergibt sich kein signifikanter Unterschied der IMT im Vergleich zu Patienten mit mehr als einer Stenose/Verschluß.

\subsection{Zusammenhang zwischen der Intima-Media-Thickness und dem Querdurchmesser}

Es zeigte sich sowohl bei Patienten mit ausschließlich karotidalen als auch bei Patienten mit ausschließlich vertebrobasilären Stenosen oder Verschlüssen ein signifikant größerer QD der ACC im Vergleich zur Kontrollgruppe (Abb. 5, Tab. 2).

Das läßt auf eine begleitende dilatative Form der Arteriosklerose bei Patienten beider Gruppen (VBS und KS) schließen. Der QD korrelierte jedoch nur gering $(\mathrm{r}=0,46$, $\mathrm{p}<0,0001$ ) mit der IMT [BOTS 97], vergleichbar mit unseren Ergebnissen.

In vorangehenden Studien über Koronararterien zeigte sich, daß der äußere QD in einem frühen Stadium der Arteriosklerose dilatierte um das Lumen und folglich den intraluminalen Druck konstant zu halten [GLAGOV 88]. Auch an den hirnversorgenden Arterien wurde bei einer IM-Verdickung der ACC eine Dilatation des äußeren QD beobachtet [BONITHON-KOPP 96, CROUSE 94, STEINKE 94].

Der äußere QD korrelierte gut mit der IMT bei POLAK et al. (1996), während der innere QD weder zu- noch abzunehmen schien.

Bei MANNAMI et al. (2000) dilatierte der innere QD mit Zunahme der IMT nicht so stark wie der äußere QD. Möglicherweise wurde dieses Ergebnis durch die zunehmende Dicke der IM-Schicht verfälscht.

BOTS et al. (1997) erkannten, daß sowohl der innere als auch der äußere QD in gleichem Maße kontinuierlich zunahm wie die IMT. Überschritt die IMT einen bestimmten Wert $(>1,10 \mathrm{~mm})$ so tendierte das Lumen sich eher zu verengen, da die IMT schneller zunahm als der QD. 
In einigen der vorangehenden Untersuchungen wurde behauptet, daß die IMT bei geringgradigen Wandverdickungen nicht Arteriosklerose sondern lediglich die Anpassung an Veränderungen der Hämodynamik mit einer Dilatation des Lumens (Scherkräfte im Blutfluß und Blutdruck) widerspiegelte [GLAGOV 88]. Erst bei höhergradigen Wandverdickungen wurde von einer Manifestation der Arteriosklerose ausgegangen [POLAK 96, BOTS 97]. Deswegen wurde vorgeschlagen, daß die IMT in Relation zum QD bewertet werden solle.

Es wurde gezeigt, daß Änderungen des Blutflusses z.B. durch IM-Verdickung zu Änderungen der Scherkräfte führen. Möglicherweise dilatiert der Gefäßdurchmesser (QD) um die ursprünglichen Scherkraftverhältnisse wiederherzustellen. Und um auch den Blutdruck konstant zu halten nimmt die IMT zu. Dadurch werden beide Faktoren im arteriellen Gefäßsystem relativ konstant gehalten. Es pendelt sich sozusagen immer wieder ein neues Gleichgewicht zwischen den beiden Größen ein [PRIES 95, LANGILLE 86, BROWNLEE 91, STARY 92].

Nicht zu vergessen ist die Wirkung der Faktoren wie Alterung der Gefäße oder Bluthochdruckstreß auf die elastischen Fasern über viele Jahre, die zu degenerativen Veränderungen und Versteifung der Arterienwände führen können [O'ROURKE 90]. POPELE et al. (2001) fanden eine positive Korrelation zwischen der IMT in der ACC und der Steifheit der arteriellen Gefäße.

Einige Untersucher konnten einen Zusammenhang zwischen Zunahme der IMT und Dilatation des Lumens nur bis zu bestimmten Grad der $\operatorname{IMT}(<1,10 \mathrm{~mm})$ erkennen [POLAK 96, BOTS 97].

Wir haben ausschließlich den inneren QD der ACC gemessen und diesen zwischen Patienten mit hämodynamisch wirksamen Stenosen/Verschlüssen und gesunden Kontrollpersonen verglichen. Möglicherweise ist das eine Ursache für die geringe Korrelation zwischen QD und IMT. Um den Zusammenhang zwischen QD und IMT noch besser zu verstehen wäre es sinnvoll beide, den inneren und äußeren QD zu betrachten.

Zusammenfassung: Sowohl Patienten mit karotidalen als auch Patienten mit vertebrobasilären Stenosen oder Verschlüssen zeigen ein größeres Lumen (QD) der ACC im Vergleich zur Kontrollpersonen. Der QD korreliert jedoch nur gering mit der IMT. 


\subsection{Querdurchmesser der A. carotis communis bei Männern und Frauen}

\section{Folgendes wurde bestätigt:}

Der durchschnittliche innere QD (Lumen) der ACC bei jüngeren gesunden Menschen beträgt etwa $5 \mathrm{~mm}$. Der äußere QD ist der Abstand zwischen den beiden Adventitiagrenzschichten, also der innere QD abzüglich zweimal der IMT. Die durchschnittliche IMT beträgt bei Gefäßgesunden ca. 0,5-0,8 mm. Viele Faktoren wie z.B. arterieller Hypertonus und Gefäßalterung können dazu beitragen, daß die Arterien dilatieren. So ist das Gefäßlumen z.B. während der Systole weiter als in der Diastole. Klinische Experimente mit Tieren zeigten, daß arterielle Gefäße bei Arteriosklerose (Zunahme der IMT) zunächst dilatierten, d.h. den Umfang vergrößerten um den ursprünglichen QD annähernd konstant zu halten [CLARKSON 81]. Dadurch resultierte weniger Lumenverengung als erwartet. Autopsiestudien von Glagov und Zarins lieferten eine qualitative Beschreibung über die Kompensationsmechanismen der menschlichen Arterien [GLAGOV 88, ZARINS 83].

In unserer Studie wurde der innere QD während der Diastole gemessen. Der durchschnittliche QD lag bei unseren älteren Patienten bei ca. 6,5 mm. In neueren Untersuchungen wurde neben der IMT auch die Dilatation der Arterien (QD) als ein adäquater prognostischer Indikator für Arteriosklerose in den Karotiden propagiert [MANNAMI 00].

In unserer Arbeit war der Unterschied des QD zwischen Männern und Frauen höchst signifikant (Tab. 7). Männer zeigten mit 6,9 mm ein sehr viel größeres Lumen als Frauen, welche nur 5,8 mm Durchmesser vorwiesen. Der innere und äußere QD in der ACC war in allen bisherigen Studien bei Männern signifikant größer als bei Frauen.

CROUSE et al. (1994) bestätigten unsere Ergebnisse. Bei männlichen Probanden wurde ein signifikant größerer innerer und äußerer QD gemessen als bei Frauen (Alter 45-64). Sie postulierten, daß die isolierte Betrachtung der IMT oder des QD die arteriosklerotische Erkrankung der Gefäße nur zum Teil beschreiben könnten und empfehlen beide Parameter in die Betrachtungen mit einzubeziehen um die Pathophysiologie besser zu erfassen.

BONITHON-KOPP et al. (1996) zeigten, daß Männer ein signifikant größeres Lumen hatten als Frauen (Alter 59-71). Es stellte sich jedoch auch heraus, daß die Körpergröße bei 
beiden Geschlechtern einen wesentlichen Einfluß auf den Querschnitt der Lumens hatte. Die Untersucher wiesen auf folgende Schwachpunkte ihrer Studie hin: 1. An der Studie nahmen nur ältere Patienten teil. 2. Der Herzzyklus wurde nicht berücksichtigt. 3. Es handelt sich um eine Querschnittstudie.

POLAK et al. (1996) fanden ebenfalls einen signifikant größeren inneren und äußeren QD bei Männern gegenüber Frauen. Sie untersuchten Männer und Frauen, die älter als 65 Jahre waren.

MANNAMI et al. (2000) beobachteten eine große Anzahl von Probanden aus der Allgemeibevölkerung. Männer hatten signifikant größere innere und äußere Diameter (QD) als Frauen.

BOTS et al. (1997) erkannten, daß sowohl der innere als auch der äußere QD in gleichem Maße kontinuierlich zunahmen wie die IMT. Überschritt die IMT einen bestimmten Wert (>1,10 mm) so tendierte das Lumen sich eher zu verengen, da die IMT schneller zunahm als der innere QD.

In den meisten aktuellen Studien wurde der Geschlechtsunterschied des Querdurchmessers bestätigt. Möglicherweise liegt der Manifestationszeitpunkt der Arteriosklerose bei Männern doch einige Jahre vor dem der Frauen. Es konnte jedoch auch bei jüngeren gesunden Probanden im Alter von 30 bis 44 Jahren ein signifikanter Geschlechtsunterschied gezeigt werden [MANNAMI 00]. Auch junge Männer hatten größere ACC-Querdurchmesser im Vergleich zu jungen Frauen.

Nicht zu vergessen ist, daß die Körpergröße der untersuchten Patienten und Probanden mit dem Durchmesser der Karotiden positiv korrelierte, d.h. größere Menschen hatten im Durchschnitt Gefäße mit größerem QD [CROUSE 94, BONITHON-KOPP 96, POLAK 96, FROST 98, PRIES 95]. Der Body-Mass-Index (BMI), welcher Gewicht und Größe berücksichtigt, korrelierte gut mit dem Durchmesser der ACC bei Männern und Frauen [BOTS 97, MANNAMI 00]. Das bedeutet, daß auch das Körpergewicht einen Einfluß auf das Kaliber der ACC nehmen kann. Schwerere Menschen hatten im Schnitt dickere Karotiden [POLAK 96]. Allgemein bekannt ist, daß Männer durchschnittlich größer und schwerer sind als Frauen. Es ist nun naheliegend zu folgern, daß Männer auch mit Karotiden von größerem QD ausgestattet sind. Folglich ist eine Begründung für den unterschiedlichen Gefäßdurchmesser zwischen den Geschlechtern möglicherweise auch in 
einer unterschiedlichen Physiologie zu suchen [BOTS 97]. Da Alter, Geschlecht, Größe und Gewicht einen bedeutenden Einfluß auf den QD der ACC haben, ist es ratsam bei statistischen Vergleichen des QD diese Parameter zu berücksichtigen.

In unserer Querschnittstudie war nur eine Momentaufnahme des Gefäßstatus möglich. Interessant dahingegen wäre es den Verlauf der Arteriosklerose (Dilatation der Karotiden) durch Längsschnittstudien zu erfassen..

Zusammenfassend findet sich, daß das Lumen (QD) der ACC bei Männern signifikant größer ist als bei Frauen.

\subsection{Intima-Media-Thickness bei Männern und Frauen}

In fast allen vergleichbaren Studien wurden die wichtigsten demographischen Daten erhoben und Frauen und Männer getrennt voneineander betrachtet.

Unsere Ergebnisse zeigten eine signifikant dickere IMT der ACC bei Männern gegenüber Frauen (Tab. 6). Beobachtet man die Arterioskleroseentwicklung und die daraus resultierenden klinischen Komplikationen wie z.B. Hirn- oder Myokardinfarkt einer gemischten Population so fällt auf, daß Männer im Schnitt früher erkranken als Frauen [ALLAN 97]. Da die IMT mit dem Alter bei Frauen und Männern kontinuierlich zunahm [FROST 98, SALONEN JT 93, BOTS 97, JENSEN-URSTAD 96, VELLER 93, ALLAN 97] vergleichbar mit unseren Ergebnissen müssen wir davon ausgehen, daß der Manifestationszeitpunkt der Arteriosklerose in der ACC bei Männern 5-10 Jahre vor dem der Frauen liegt [ROSFORS 98].

Unsere Ergebnisse bestätigen die Resultate der im folgenden zitierten Autoren:

BONITHON-KOPP (1996), O'LAERY (1992), ROSFORS (1998) und BALDASSARRE et al. (2000) untersuchten Männern und Frauen mittleren bis höheren Alters und sahen eine signifikant erhöhte IMT in der ACC bei männlichen Probanden gegenüber weiblichen Probanden.

ALLAN et al. (1997) beobachteten Probanden im Alter zwischen 60 und 80 Jahren und unterteilten sie in fünf Altersgruppen (60-64/65-69/70-74/75-80/60-80). Männer zeigten in allen fünf Gruppen signifikant höhere IMT-Werte als Frauen.

HOWARD et al. (1993) fanden beim Vergleich der IMT in der ACC zwischen Männern und Frauen mittleren Alters eine signifikant stärkere Zunahme der IMT pro Jahr bei 
männlichen weißen Probanden gegenüber weiblichen weißen Probanden. Auch bei schwarzen Studienteilnehmern konnte der Geschlechtsunterschied bestätigt werden. Jedoch zeigte die erwartete Zunahme der IMT sowohl bei Männern als auch bei Frauen insgesamt niedrigere Werte als bei den weißen Teilnehmern.

FROST et al. (1998) stellten bei 20 jüngeren Probanden die Karotiden dar und fanden, daß bei jüngeren Gesunden die IMT der Karotis zwar abhängig vom Alter und von der Körpergröße war, jedoch nicht vom Geschlecht. Die von einigen Untersuchern gefundenen Geschlechtsunterschiede können wahrscheinlich auch auf Größenunterschiede zurückgeführt werden. Deshalb sollte vor allem bei epidemiologischen Studien der Einfluß von Größe auf die IMT nicht vernachlässigt werden.

Insgesamt ist jedoch zu berücksichtigen, daß an unserer Studie im Durchschnitt ältere Patienten (Mittelwert 64,5 Jahre) teilnahmen und 90\% der Teilnehmer ein erhöhtes Risikofaktorenpotential aufwiesen. Alle Probanden wurden aus einer Fachklinik rekrutiert. In einigen Studien mit jungen gesunden Probanden aus der Allgemeinbevölkerung [FROST 98] konnten keine geschlechtsspezifischen Unterschiede der IMT festgestellt werden. Die IMT der Karotis wurde schon häufig bei älteren symptomatischen Männern und Frauen gemessen. Es wurden bisher jedoch nur wenige Untersuchungen bei gesunden jungen Probanden durchgeführt. In Anbetracht der unterschiedlichen Ergebnisse bei jungen bzw. älteren Probanden wäre es sinnvoll in folgenden Studien asymptomatische Männer und Frauen im Alter von 18 bis 35 Jahren unter die Lupe zu nehmen.

Die Frage nach den Ursachen dieser unterschiedlichen Entwicklungen zwischen Männern und Frauen wurden bisher jedoch nur zum Teil beantwortet. Genetische Disposition, ethnische Unterschiede, sozioökonomische Aspekte, differentes soziales Rollenverhalten (Streß) sowie ein unterschiedliches Risikofaktorenprofil sind weitere mögliche Ursachen für diese Diskrepanz [MATTHEWS 98, D'AGOSTINO 96, DUGGIRALA 96] (s. Kap. 5.8).

\section{Zusammenfassend haben Männer eine signifikant dickere IM-Schicht als Frauen.}




\subsection{Intima-Media-Thickness der rechten und linken A. carotis communis}

In den meisten veröffentlichten Studien wurden die beiden Karotiden (links und rechts) nicht getrennt voneinander untersucht oder es wurde berichtet, daß die IMT auf beiden Seiten vergleichbar sei [SOLBERG 71].

In unserer Studie zeigte die IMT linksseitig tendenziell höhere Werte als rechtsseitig, auch wenn Männer und Frauen getrennt voneinander untersucht wurden. Dieser Unterschied war jedoch nicht signifikant (Tab. 6). Wir müssen nach unseren Ergebnissen davon ausgehen, daß die IMT auf beiden Seiten vergleichbar ist. In einigen vorangehenden sonographischen Untersuchungen wurde jedoch eine unterschiedliche IMT der beiden Seiten festgestellt.

ROSFORS et al. (1998) gaben in ihrer Untersuchung eine signifikant erhöhte IMT der linken ACC im Vergleich zur rechten Seite an, jedoch nur bei Männern [ALLAN 97].

JENSEN-URSTAD et al. (1996) untersuchten 59 Patienten (Durchschnittsalter 44 Jahre), welche alle an Diabetes mellitus (IDDM) erkrankt waren. 31 Patienten erhielten eine intensivierte konventionelle Langzeitinsulinbehandlung (HbA1c 7,1). 28 Patienten wurden mit Standardinsulin (HbA1c 8,2) behandelt. Die Patienten mit der Standardinsulinbehandlung zeigten eine signifikant erhöhte IMT der linken Karotis im Vergleich zur rechten.

LEMNE et al. (1995) beobachteten 161 Männer im Alter von 35 bis 55 Jahren. 81 Patienten litten an einem arteriellen Bluthochdruck (Borderline-Hypertension). 80 Probanden bildeten die Kontrollgruppe. In beiden Gruppen war die IMT linksseitig zwar nur tendenziell aber signifikant erhöht gegenüber rechtsseitig. Beim Vergleich zwischen den beiden Gruppen fiel auf, daß nur in der rechten Karotis eine signifikante Zunahme der IMT gegenüber der Kontrollgruppe zu verzeichnen war [BOTS 93].

Beim Vergleich zwischen Patienten mit Borderline-Hypertension und gesunden Probanden sahen LEMNE et al. (1995) eine deutliche Zunahme der IMT in der rechten A. carotis communis bei den an Bluthochdruck erkrankten Studienteinehmern. Eine mögliche Erklärung für die unterschiedliche Arterioskleroseprogression ist, daß sich arteriosklerotische Läsionen an der linken Karotis früher manifestieren. Da die linke ACC direkt aus dem Aortenbogen entspringt, kommt es hier im Vergleich zur rechten Seite auch zu erhöhten Scherkräften im Blutfluß durch Verwirbelungen. In vorangehenden Arbeiten 
wurde schon gezeigt, daß die Wirkung der Scherkräfte die Lokalisation und den Grad der Arteriosklerose beeinflussen [ZARINS 83]. Bei Patienten mit Risikofaktoren (z.B. Arterieller Hypertonus), wie in der Untersuchung von LEMNE et al. (1995), scheint der Arterioskleroseprozeß systemisch fortzuschreiten bis der Unterschied der IMT zwischen linker und rechter Karotis nicht mehr klar erkennbar war.

An unserer Studie nahmen ältere Patienten teil (64,5 $\pm 12,5$ Jahre). 90\% der Untersuchten hatten mindestens einen Risikofaktor. Es ist anzunehmen, daß bei unseren Patienten die Arteriosklerose generalisiert fortgeschritten war und deshalb die IMT im Seitenvergleich keinen signifikanten Unterschied mehr zeigte. Natürlich ist es unmöglich aus unserer aktuellen Querschnittsstudie definitive Schlußfolgerungen zu ziehen. Aber einige vorangehende Untersuchungen bestätigen unsere Ergebnisse [BOTS 93]. Durch unsere Ergebnisse wird nochmals deutlich, daß es wichtig ist die IMT in beiden Karotiden, der rechten und der linken, zu messen.

\section{Zusammenfassung: Die IMT der rechten und linken ACC verhält sich nicht signifikant unterschiedlich.}

\subsection{Seitenverteilung der Stenosen und Verschlüsse}

Die Arteriosklerose kann sich in allen Abschnitten der Arterien manifestieren, sowohl in Arterien vom elastischen Typ als auch in Arterien vom muskulären Typ. Aber einige Gefäßregionen sind hauptsächlich betroffen, während andere wiederum fast ausgespart werden. Die Karotisgabel, die Koronararterien, die infrarenale Bauchaorta und Arterien, welche die unteren Extremitäten versorgen, sind Prädilektionsstellen. Die Neigung zur Plaquebildung in Gefäßgabelungen und Gefäßkrümmungen haben vermuten lassen, daß lokal bedingte mechanische Faktoren wie z.B. Fußturbulenzen (Scherkräfte) und intraluminaler Druck die Entstehung der Arteriosklerose verstärken. Studien über Gefäße mit erhöhtem Risiko am Menschen haben quantitative Beweise geliefert, daß Plaques/Stenosen dort auftreten, wo Flußgeschwindigkeit vermindert ist und der Fluß von einer laminaren Strömung abweicht [GLAGOV 88, ZARINS 83, PEARSON 84].

Die linke ACC entspringt direkt aus dem Aortenbogen und unterscheidet sich damit von der rechten ACC. Möglicherweise sind die unterschiedlichen anantomischen Verhältnisse 
in beiden Karotiden ein Grund, weshalb Plaques/Stenosen häufiger auf der linken Seite zu sehen sind [LEMNE 95].

In unserer Studie zeigten sich bei Patienten aus allen Stenosegruppen stets mehr hämodynamisch wirksame Makroangiopathien auf der linken Seite (Tab 8). Von 142 mittelgradigen Stenosen der hirnversorgenden Arterien waren 83 links und 53 rechts gelegen. Dies bestätigt eine seitenunterschiedliche Entwicklung der Arteriosklerose.

ROSFORS et al. (1998) sahen eine Korrelation zwischen IMT und Plaques/Stenosen in der Karotisgabel nur auf der linken Seite. Echodichte verkalkte Plaques, welche Stenosen verursachen, waren hauptsächlich linksseitig $\mathrm{zu}$ finden. Und fünf von sieben Karotisverschlüssen waren links lokalisiert.

LEMNE et al. (1995) begründeten die frühere Entstehung der Arteriosklerose in den linken Karotiden mit den unterschiedlichen anatomischen Verhältnissen. Denn die linke ACC entspringt direkt aus dem Aortenbogen, wodurch im Vergleich zur rechten ACC unterschiedliche hämodynamische Verhältnisse herrschen [ZARINS 83].

Die Ergebnisse von HOWARD et al. (1994) unterstützen die Hypothese, daß es eine systemische, eine anatomische und eine fokale Form der Arteriosklerose gibt. Sie zeigten, daß eine Zunahme der IMT an einer Stelle in den Karotiden mit der Zunahme der IMT an einer beliebigen anderen Stelle in den Karotiden (z.B. kontralaterale ACC) signifikant korrelierte. Das läßt eine systemische Natur der Arteriosklerose vermuten. Eine höhere Korrelation ergab sich, wenn man die ipsilaterale ACI mit dem ipsilateralen Flußteiler verglich in Relation zur ipsilateralen ACC. Dieses Ergebnis beleuchtet die bevorzugte Entstehung von Plaques/Stenosen in und oberhalb der Karotisgabel, was eine anatomische Form der Arteriosklerose erkennen läßt. Es ergaben sich jedoch auch zahlreiche Diskrepanzen der IMT, wenn man die IMT an verschiedenen Stellen miteinander verglich. Diese Erkenntnis läßt auch dieVermutung einer fokalen Form der Arteriosklerose zu.

Möglicherweise spielen auch Unterschiede in der histologischen Struktur der Arterienwände eine Rolle in der Entstehung von Arteriosklerose. So ist z.B. die A. carotis interna, eine Prädilektionsstelle der extrakraniellen karotidalen Halsgefäße, histologisch anders beschaffen als die A. carotis communis [HEATH 73]. 
In unserer Arbeit wurde die IMT der ACC auf beiden Seiten gemessen. In Anbetracht der Prädilektiosstellen für Arteriosklerose, welche in den distalen Abschnitten der Karotiden (Bifurkation und ACI) zu finden sind, empfahlen HOWARD et al. (1994) aufgrund höherer Genauigkeit die IMT in sechs Abschnitten der Karotiden zu messen (ACC/Bif./ACI jeweils auf beiden Seiten). Zusätzlich würde diese Mehrfachmessung der fokalen bzw. anatomischen Form der Arteriosklerose Rechnung tragen.

In unserer Arbeit waren deutlich mehr Makroangiopathien auf der linken Seite der hirnversorgenden Arterien $\mathrm{zu}$ finden. Schon in zahlreichen Studien wurde eine Seitenbevorzugung der linken Karotis beschrieben [ROSFORS 98, ALLAN 97, JENSENURSTAD 96].

Zusammenfassung: Stenosen oder Verschlüsse in den hirnversorgenden Arterien sind häufiger auf der linken Seite zu finden.

\subsection{Zerebrovaskuläre Risikofaktoren}

Die zerebrovaskulären Risikofaktoren bewirken eine kontinuierliche Zunahme der IMT der ACC [EBRAHIM 99, HOWARD 97, MANNAMI 97]. Mit zunehemndem Lebensalter werden in die Intima Lipide abgelagert. Makrophagen wandern ein und die Intima verdickt sich. Begünstigt wird dieser Prozeß durch fettreiche Ernährung, Rauchen, Bluthochdruck und Stoffwechselerkrankungen wie z.B. Zuckerkrankheit.

In unserer Arbeit wurden die klassischen zerebrovaskulären Risikofaktoren aller Studienteilnehmer erfragt und der Zusammenhang mit der IMT geprüft. $90 \%$ der Untersuchten hatten mindestens einen der folgende Risikofaktoren: Arterieller Hypertonus, Hypercholesterinämie, Diabetes mellitus, Nikotinabusus, Familienanamnese, vorangehende koronare und zerebrale Ereignisse.

Unsere Ergebnisse zeigten bei allen Patienten mit den oben genannten Risikofaktoren eine erhöhte IMT im Vergleich zu Patienten ohne Risikofaktor mit Ausnahme der Personen, die rauchten. Bei Rauchern war die IM dünner als bei Nicht-Rauchern. Studienteilnehmer mit Nikotinabusus waren jedoch durchschnittlich zehn Jahre jünger als Nicht-Raucher. Signifikant war dieser Unterschied nur bei Studienteilnehmern mit Arteriellen Hypertonus, 
Diabetes mellitus und bei Rauchern (Tab. 9). Das Alter der Studienteilnehmer korrelierte gut mit der IMT und dem QD (Abb. 13 u. 14).

Eine hohe Korrelation zwischen Alter und IMT wurde schon in zahlreichen Studien mit Patienten/Probanden im mittleren Alter zwischen 40 und 50 Jahren [LEMNE 95, JENSENURSTAD 96, JENSEN-URSTAD 97] und ebenso bei älteren Patienten/Probanden zwischen 60 und 70 Jahren beobachtet [ADAMS 95, ALLAN 97, BONITHON-KOPP 96]. HOMMA et al. (2001) fanden eine lineare Zunahme der IMT mit dem Alter in allen Altersgruppen (21-105 Jahre). In unserer Studie mit älteren Patienten (Mittelwert 64,5 Jahre) war das Alter ein wichtiger Faktor, der die IMT beeinflußte.

BALDASSARRE et al. (2000) untersuchten Männer und Frauen mittleren Alters (Mittelwert 54,6 Jahre) und sahen einen signifikanten Zusammenhang zwischen der IMT und dem systolischen Blutdruck, der Blutglukose, dem Gesamtcholesterin und den Triglyzeriden [HUGHES 93, WENDELHAG 92, SUURKÜLA 94]. Auch nach Unterteilung in drei Altersklassen und Berücksichtigung des Alters (Korrelation nach Spearman) blieb dieser Zusammenhang statistisch signifikant. Die IM-Schicht war dicker bei Hypertonikern als bei Nicht-Hypertonikern [BOTS 93, LEMNE 95, SU 01] (Abb. 10). Im Gegensatz zu unseren Ergebnissen (Abb. 11) wurde zwischen Diabetikern und NichtDiabetikern kein signifikanter Unterschied der IMT gefunden.

BALDASSARRE et al. (2000) vermuteten, daß die hohe Prävalenz der Patienten mit Hypercholesterinämie die Effekte des Diabetes mellitus auf die IMT der Karotis möglicherweise maskierten [KAWAMORI 92, NISKANEN 96].

JENSEN-URSTAD et al. (1996) untersuchten 59 Patienten mittleren Alters (44 Jahre), welche alle an Diabetes mellitus (IDDM) erkrankt waren. 31 Patienten erhielten eine intensivierte konventionelle Langzeitinsulinbehandlung (HbA1c 7,1). 28 Patienten wurden mit Standardinsulin (HbA1c 8,2) behandelt. Die Patienten mit der Standardinsulinbehandlung zeigten eine signifikant erhöhte IMT im Vergleich zu den Patienten, die sich einer intensivierten Langzeitinsulinbehandlung unterzogen.

In vielen vorangehenden Studien wurde ein statistisch signifikanter Zusammenhang zwischen den klassischen kardiovaskulären Risikofaktoren wie z.B. Diabetes mellitus, Arterieller Hypertonus, Nikotinabusus, Gesamtcholesterin und einer Zunahme der IMT 
bzw. Arterioskleroseausprägung (Plaques/Stenosen) in der Karotis nachgewiesen [SALONEN R 91, VELLER 93, MANNAMI 97].

Andere Arbeitsgruppen berichteten, daß bei älteren Patienten die konventionellen kardiovaskulären Risikofaktoren keine signifikante Verdickung der IM bewirkten:

Das Ziel der Studie von FABRIS et al. (1994) war die Ausprägung der Arteriosklerose in den Karotiden (Anzahl der Plaques, Grad der Stenosen) und die Relation zu den konventionellen kardiovaskulären Risikofaktoren in verschiedenen Altersgruppen (18-44, 45-64, 65-74, $\geq 75$ Jahre) zu bemessen. Alter, Nikotinabusus, und Gesamtcholesterin korrelierten zunächst gut mit der Zunahme von arteriosklerotischen Läsionen (IMT) in allen Altersgruppen. Nach einer schrittweisen Regressionsanalyse mit Berücksichtigung des Alters fiel auf, daß bei den älteren Patienten ( $\geq 65$ Jahre) Nikotinabusus und Gesamtcholesterin nicht länger mit der Arteriosklerose in der Karotis zusammenhing. JUNGQUIST et al. (1991) und PUJIA et al. (1992) konnten bei älteren Patienten ebenfalls keine statistisch signifikante Beziehung zwischen Risikofaktoren und dem Grad der Arteriosklerose finden.

Auch in unserer Studie mit älteren Patienten und Probanden (Mittelwert 64,5 Jahre) konnten einige der konventionellen Risikofaktoren (Hypercholesterinämie, Familienanamnese und vorangehende koronare/zerebrale Ereignisse) nicht signifikant mit der IMT korrelieren (Tab 9). Das Alter der Studienteilnehmer zeigte jedoch eine gute Korrelation mit der IMT (Abb. 13), d.h. mit zunehmenden Alter zeigten die Patienten und Probanden auch eine dickere IM.

Möglicherweise sind in Gruppen mit älteren Studienteilnehmern ( $\geq 65$ Jahre) vergleichbar mit unserer Querschnittstudie Patienten mit einem hohen zerebrovaskulären Risiko unterrepräsentiert. Denn diese versterben häufig schon vor dem Alter an kardiologischen Komplikationen. Um dieses Ungleichgewicht zu vermeiden wäre es sinnvoll auch jüngere Patienten mit kardiovaskulären Risikofaktoren in Verlaufsstudien zu untersuchen.

Zusammenfassend kann man sagen, daß Hypertoniker und Diabetiker eine dickere IM-Schicht aufweisen als Nicht-Hypertoniker und Nicht-Diabetiker. Das Alter korreliert gut mit der IMT. 
Die IMT der ACC sei ein prognostischer Faktor für arteriosklerotische Läsionen auch in Gefäßregionen außerhalb der hirnversorgenden Arterien wie z.B. Koronar- und Femoralarterien [CRAVEN 90, WENDELHAG 93, BURKE 95, ALLAN 97, BOTS 97]. Die Zunahme der IMT bei älteren Patienten ohne kardiovaskulärer Vorgeschichte hing direkt mit einem erhöhten Risiko für Herz- und Hirninfarkte zusammen. Folglich wurde von O'LEARY et al. (1999) vorgeschlagen die IMT in die Liste der zerebrovaskulären Risikofaktoren aufzunehmen. Sie untersuchten die Beziehung zwischen der IMT und der Inzidenz von Herz- oder Hirninfarkten.

Unsere Ergebnisse zeigten, daß Patienten mit vorangehenden koronaren oder zerebralen Ereignissen wie z.B. Herz- oder Hirninfarkte zwar tendenziell höhere IMT-Werte hatten im Vergleich zu Patienten ohne vorherige vaskuläre Ereignisse. Dieser Unterschied war aber nicht signifikant (Tab. 9). Wir müssen also nach unseren Ergebnissen davon ausgehen, daß die IMT bei Patienten mit vorangehenden zerebralen bzw. kardialen Ereignissen und Kontrollpersonen vergleichbar ist.

ADAMS et al. (1995) bestätigten unsere Ergebnisse. Sie fanden bei älteren Patienten nur eine sehr schwache signifikante Korrelation zwischen der IMT und dem Ausmaß der koronaren Herzerkrankung. Das Ausmaß der KHK wurde angiographisch dokumentiet. Sie folgerten, daß die IMT als klinischer Parameter zur Identifizierung von Patienten mit oder ohne koronarer Herzerkrankung nicht spezifisch und sensitiv genug sei und deshalb in der Praxis nicht sinnvoll einsetzbar sei. Alter, männliches Geschlecht und Diabetes mellitus hingegen korrelierten sehr viel signifikanter mit dem Ausmaß der KHK als die IMT selbst. Eventuell haben die traditionellen Risikofaktoren einen unterschiedlichen Effekt auf die verschiedenen Gefäßsysteme wie Karotiden oder Koronararterien [OLSSON 91].

LUDWIG et al. (1993) z.B. stellten heraus, daß Hypercholesterinämie einen stärkeren Einfluß auf die Enstehung von Atheromen in den Herzkranzgefäßen zeigte als auf die Entstehung von Arteriosklerose in den Halsschlagadern. Und BLANKENHORN et al. (1994) beobachteten, daß Atherome in der Karotis häufig später im Leben auftraten als Plaques in den Koronararterien. Möglicherweise ist die unterschiedliche Wirkung einiger Risikofaktoren auf die verschiedenen Gefäßsysteme wie Karotiden (IMT) oder Koronararterien (KHK) ein Grund für die fehlende Korrelation zwischen diesen beiden Parametern für Arteriosklerose. 
Andere Autoren wiederum sahen einen deutlichen Zusammenhang zwischen der IMT und dem Ausmaß der KHK. Sie postulieren die IMT als reliablen Parameter zur Vorhersage des kardiovaskulären Risikos auch in einem frühen Stadium der Arteriosklerose.

SALONEN JT et al. (1991) untersuchten Männer mittleren Alters (42-60 Jahre) in einer Verlaufsstudie. Sie verglichen die Morphologie in den Karotiden sonographisch (Normal < IM-Verdickung < Plaques < Stenosen) mit dem klinischen Ausmaß der KHK (kein < möglicher < gesicherter Herzinfarkt) und fanden eine enge Beziehung zwischen der karotidalen Gefäßwandmorphologie und der KHK. Schon in den 60er Jahren wurde dieser Zusammenhang in pathologischen Studien erstmals erwähnt [MATHUR 63, YOUNG 60].

PERSSON et al. (1994) schätzten das Risiko für zukünftige koronare Ereignisse, indem sie für jeden Patienten ein individuelles Risikofaktorenprofil nach Framingham [KANNEL 76] erstellten. Beinhaltet waren Risikofaktoren wie Alter, Rauchgewohnheiten, Blutdruck, Glukosetoleranz und LDL-Cholesterin. Die IMT nahm in gleichem Maße zu wie das Risiko für zukünftige koronare Ereignisse.

Es fällt jedoch auf, daß in den beiden zuletzt genannten Studien das Ausmaß der KHK nicht angiographisch bestimmt wurde wie bei ADAMS et al. (1995). SALONEN (1991) und PERSSON (1994) et al. definierten das Ausmaß der KHK nach klinischen Kriterien wie Inzidenz von Herzinfarkten oder Risikofaktorenprofil. Und beide Autoren sahen im Gegensatz zu ADAMS et al. (1995) einen starken signifikanten Zusammenhang zwischen der IMT/Plaques der hirnversorgenden Arterien und dem Ausmaß der KHK. Möglicherweise waren die unterschiedlichen Methoden ein Grund für die schwache Korrelation zwischen IMT und KHK bei ADAMS et al. im Vergleich zu SALONEN und PERSSON et al.

Wir definierten das Ausmaß der KHK wie SALONEN und PERSSON et al. nach klinischen Kriterien (s. Kap 3.4.1.). Unsere Ergebnisse zeigten aber keine signifikante Korrelation zwischen der IMT und dem Ausmaß der KHK. Insgesamt ist es jedoch problematisch die verschiedenen Ergebnisse miteinander zu vergleichen, da die Methoden (z.B. Definition des Ausmaßes der KHK) in den oben genannten Studien stark differieren. 
Nach unseren Ergebnissen müssen wir davon ausgehen, daß die IMT als prognostischer Parameter zur Vorhersage von kardialen oder zerebralen Ereignissen in der Klinik nicht sinnvoll einsetzbar ist.

Aufgrund der klinischen Bedeutung dieser Aussage (Vorhersage von koronaren/zerebralen Ereignissen) sind noch weitere Verlaufsstudien nötig, die den Zusammenhang zwischen der IMT und akuten klinischen Ereignissen wie z.B. Herz- und Hirninfarkt bestätigen.

Zusammenfassung: Nach unseren Ergebnissen müssen wir davon ausgehen, daß die IMT bei Patienten mit vorangehenden zerebralen bzw. kardialen Ereignissen und bei Kontrollpersonen vergleichbar ist.

In aktuellen Untersuchungen wurden neben den klassischen kardiovaskulären Risikofaktoren immer wieder neue Faktoren beschrieben, welche einen Einfluß auf die IMT zeigten.

DUGGIRALA et al. (1996) z.B. studierten die genetische Basis als Ursache für eine unterschiedliche IMT. Sie untersuchten einige Großfamilien in Mexico Stadt und fanden eine hohe Erblichkeit der IMT innerhalb der einzelnen Familien.

Ethnische Unterschiede der IMT beschrieben D'AGOSTINO et al. (1996) zwischen weißen, spanischstämmigen und schwarzen US-Amerikanern. So hatten Schwarze eine dickere IMSchicht als Weiße. Die Spanischstämmigen zeigten die geringste IMT [HOWARD 93].

Einwohner der karibischen Inseln afrikanischen Ursprungs, welche nach Großbritannien ausgewandert waren, hatten ein erhöhtes Schlaganfallrisiko und eine dickere IM-Schicht als weiße Briten, auch wenn man die klassischen zerebrovaskulären Risikofaktoren berücksichtigte. Es zeigten sich höchst signifikante ethnische Unterschiede in der Verteilung vieler wichtiger Gene für den zerebrovaskulären Phänotyp zwischen den Volksgruppen. Obwohl die untersuchten Gene in dieser Studie die ethnischen Unterschiede nicht signifikant erklärten, könnten andere genetische Prädispositionen oder Umwelteinflüsse (z.B. Clamydia pneumoniae etc.) Ursache für diese Differenzen sein [MARKUS 01].

Der sozioökonomische Status ( z.B. Bildung und Einkommen) war mit der Morbidität und Mortalität von Herz- und Kreislauferkrankungen eng verknüpft. So zeigten LYNCH et al. 
(1997), daß Männer mit niedrigem Bildungsstand und geringem Einkommen eine dickere IM-Schicht hatten als gebildete Männer mit hohem Einkommen.

Auch mentaler Streß (z.B. Sprechen vor Publikum) kann zu einer Verdickung der IMSchicht führen. So sahen MATTHEWS et al. (1998) während der Streßexposition eine Zunahme des Blutdrucks und der IMT. CASTILLO-RICHMOND et al. (2000) beobachteten eine Abnahme der IMT bei Stressverminderung durch transcendentale Meditation.

Umwelteinflüsse wie z. B. die Kontamination mit Bakterien oder Viren wurden immer wieder für die Entstehung von Arteriosklerose und deren Folgen verantwortlich gemacht. Zellkörper von Bakterien der Spezies Clamydia pneumoniae wurden z.B. in arteriosklerotischen Läsionen entdeckt. SCHMIDT et al. (2000) beobachteten bei erhöhten Antikörpertitern für Clamydia pneumoniae eine Verdickung der IM-Schicht.

Die IMT wird von vielen Faktoren beeinflußt. Nicht nur die klassischen zerebrovaskulären Risikofaktoren sondern auch Faktoren wie genetische Disposition, ethnische Unterschiede, sozioökonomische Aspekte und Umwelteinflüsse können eine Zunahme der IMT bewirken.

\subsection{Methodenkritik zum Studiendesign und zur Studiengröße}

Es handelt sich um eine monozentrische Querschnittstudie. 121 konsekutive Patienten und Probanden wurden im Zeitraum von 08.97 bis 08.98 untersucht. Patienten mit isolierten vertebrobasilären Stenosen oder Verschlüssen $(n=16)$ waren äußerst selten. Sehr viel häufiger waren Patienten mit rein karotidalen Stenosen oder Verschlüssen $(n=57)$ zu finden. Beim Vergleich der IMT zwischen Patienten mit VBS- und KS-Makroangiopathie zeigte sich kein signifikanter Unterschied. Möglicherweise ist die geringere Fallzahl der Studienteilnehmer mit ausschließlich vertebrobasilären Makroangiopathien ein Grund für die fehlende Signifikanz. Mit unserem Ergebnis " Patienten mit VBS-Makroangiopathien haben tendentiell eine geringere IMT als Patienten mit KS-Makroangiopathien" konnte jedoch eine Tendenz aufgezeigt werden. Bei unserer Untersuchung handelt es sich um eine Beobachtungsstudie zum Kollektiv. Es wäre denkbar und sinnvoll eine multizentrische 
Studie mit 100 bis 1000 Studienteilnehmern zu entwerfen um ein signifikantes Ergebnis zu erhalten. 


\section{Literaturverzeichnis}

1. Adams MR, Nakagomi A, Keech A, Robinson J, McCredie R, Bailey BP, Freedman B, Celermajer DS (1995) Carotid intima-media thickness is only weakly correlated with extend and severity of coronary artery disease. Circulation 92:2127-2134

2. Allan PL, Mowbray PI, Lee AJ, Fowkes GR (1997) Relationship between carotid intima-media-thickness and symptomatic and asymtomatic peripheral arterial disease. Stroke 28:348-353

3. Baldassarre D, Amato M, Bondioli A, Sirtori CR, Tremoli E (2000) Carotid intimamedia-thickness measured by ultrasongraphy in normal clinical practice correlates well with atherosclerosis risk factors. Stroke 31:2426-2430

4. Blankenhorn DH, Selzer RH, Crawford DW, Barth JD, Liu CR, Liu CH, Mack WJ, Alaupovic P (1993) Beneficial effects of colestipol-niacin therapy on the common carotid artery: two- and four-year reduction of intima-media thickness measured by ultrasound. Ciculation 88:20-28

5. Bond MG, Barnes RW, Riley WA, Wilmoth SK, Chambless LE, Howard G, Owens B (1991) High-resolution B-mode ultrasound scanning methods in the atherosclerosis risk communities study (ARIC). J Neuroimag 1:68-73

6. Bonithon-Kopp C, Jouven X, Taquet A, Touboul PJ, Guize L, Scarabin PY (1993) Early atherosclerosis in healthy middle-aged women. A follow-up study. Stroke 24:1837-1843

7. Bonithon-Kopp C, Touboul PJ, Berr C, Magne C, Ducimetière P (1996) Factors of carotid arterial enlargement aged 59 to 71 years. Stroke 27:654-660

8. Bots ML, Hofman A, Grobbee DE (1997) Increased common carotid intima-media thickness - Adaptive response or a reflection of atherosclerosis ? Stroke 28:2442-2447

9. Brownlee RD, Langeville BL (1991) Arterial adaptations to altered blood flow. Can J Physiol Pharmacol. 69:978-983 
10. Burke GL, Evans GW, Riley WA, Sharrett AR, Howard G et al. (1995) Arterial wall thickness is associated with prevalent cardiovascular disease in middle aged adults (ARIC). Stroke 26:386-391

11. Castillo-Richmond A, Schneider RH, Alexander C, Cook R, Myers H, Nidich S, Haney C, Rainforth M, Salerno J (2000) Effects of stress reduction on Carotid Atherosclerosis in hypertensive african americans. Stroke 31:568

12. Chambless LE, Zhong MM, Arnett D, Folsom AR, Riley WA, Heiss G (1996) Variability in B-mode ultrasound measurements in the atherosclerosis risk in communities (ARIC) study. Ultrasound in Med. \& Biol. 22:545-554

13. Clarkson TB, Bond MG, Bullock BC, Marzetta CA (1981) A study of atherosclerosis regression in Macaca mulatta. Exp Mol Pathol. 34:345-368

14. Craven TE, Ryu JE, Espeland MA, Kahl FR, McKinney WM, Toole JF, McMahan MR, Thompson CJ, Heiss G, Crouse JR (1990) Evaluation of the association between carotid artery atherosclerosis and coronary artery stenosis. Circulation 82:1230-1242

15. Crouse JR, Goldbourt U, Evans G, Pinsky J, Sharrett AR, Sorlie P, Riley W, Heiss G (1994) Arterial enlargement in the atherosclerosis risk in communities (ARIC) cohort. Stroke 25:1354-1359

16. Crouse JR, Goldbourt U, Evans G, Pinsky J, Sharrett AR, Sorlie P, Riley W, Heiss G (1996) Risk factors and segment-specific carotid arterial enlargement in the atherosclerosis risk in communities (ARIC) cohort. Stroke 27:69-75

17. D'Agostino RB, Burke G, O'Leary D, Rewers M, Selby J, Savage PJ, Saad MF, Bergman RN, Howard G, Wagenknecht L, Haffner SM (1996) Ethnic differences in carotid wall thickness. Stroke 27:1744-1749

18. Duggirala R, Villalpando CG, O'Leary DH, Stern MP, Blangero J (1996) Genetic basis of variation in carotid artery wall thickness. Stroke 27:833-837

19. Ebrahim S, Papacosta O, Whincup P, Wannamethee G, Walker M, Niclaides AN et al. (1999) Carotid plaque, intima-media thickness, cardiovascular risk factors and prevalent cardiovascular disease in men and women. Stroke 30:841-850 
20. Fabris F, Zanocchi M, Bo M, Fonte G, Poli L, Bergoglio I, Ferrario E, Pernigotti L (1994) Carotid plaque, aging, and risk factors. Stroke 25:1133-1140

21. Fox B, James K, Morgan B (1982) Distribution of fatty and fibrous plaques in young human coronary arteries, 41:337-347

22. Frost D, Friedl A, Beischer W (1998) Bestimmung der Intima-Media-Dicke an der Arteria carotis: methoden-, probanden- und untersucherabhängige Einflüsse. Ultraschall in Med. 19:168-173

23. Geroulakos G, O'Gorman DJ, Kalodiki E, Sheridan DJ, Nicolaides AN (1994) The carotid intima-media thickness as a marker of the presence of severe symptomatic coronary artery disease. Eur. Heart J 15:781-785

24. Glagov S, Zarins C, Giddens DP, Ku DN (1988) Hemodynamics and Atherosclerosis. Arch Pathol Lab Med 112:1018-1031

25. Grobbee DE, Bots ML (1994) Carotid artery intima-media thickness as an indicator of generalized atherosclerosis. J. Int. Med. 236:567-573

26. Heath D, Smith P, Harris P, Winson M (1973) The atherosclerosis human carotid sinus. J. Path. Vol. 110

27. Heiss G, Sharrett AR, Barnes R, Chambless LE, Szklo M, Azola C (1991) Carotid atherosclerosis measured by B-mode ultrasound in populations: association with cardiovascular risk factors in the ARIC study. Am J Epidemiol. 134:250-256

28. Hennerici M, Rautenberg W, Trockel U, Kladetzky RG (1985) Spontaneous progression and regression of small carotid atheroma. Lancet 1:1415-9

29. Homma S, Hirose N, Ishida H, Ishii T, Araki G (2001) Carotid plaque and IntimaMedia Thickness assessed by B-mode ultrasonography in subjects ranging from young adults to centenarians. Stroke 32:830

30. Howard G, Burke GL, Evans GW, Crouse JR, Riley W, Arnett D, Lacy R de, Heiss G (1994) Relations of intimal-medial thickness among sites within the carotid artery as evaluated by B-mode ultrasound. Stroke 25:1581-1587 
31. Howard G, Sharrett AR, Heiss G, Evans GW, Chambless LE, Riley WA, Burke GL (1993) Carotid artery Intimal- Medial Thickness in general populations as evaluated by B-mode ultrasound. Stroke 24:1297-1304

32. Hughes AD, Sinclair AM, Geroulakos G, Mayet J, Mackay J, Shahi M, Thom S, Nicolaides A, Sever PS (1993) Structural changes in the heart and carotid arteries associated with hypertension in humans. J Hum Hypertens. 7:395-397

33. James EM, Earnest F, Forbes GS, Reese DF, Houser OW, Folger WN (1982) Highresolution dynamic ultrasound imaging of the carotid bifurcation: A prospective evaluation. Radiology 144:853-858

34. Jensen-Urstad KJ, Reichard PG, Rosfors JF, Lindblad LE, Jensen-Urstad MT (1996) Early atherosclerosis is retarded by improved long-term blood glucose control in patients with IDDM. Diabetes, Vol. 45

35. Jungquist G, Hanson BS, Isacsson SO, Janzon L, Stehen B, Lindell SE (1991) Risk factors for carotid artery stenosis: an epidemiological study of men aged 69 years. $\mathrm{J}$ Clin Epidemiol Vol. 44, No. 4/5, pp. 347-353

36. Kannel WB, McGee D, Gordon T (1976) A general cardiovascular risk profile. The Framingham study. Am J Cardiol. 38:46-51

37. Kanters S, Algra A, Leeuwen MS van, Banga JD (1997) Reproducibility of in vivo carotid intima-media thickness measurements: A review. Stroke 28:665-671

38. Kawamori R, Yamasaky Y, Matsushima H, Nishizawa H, Nao K, et al. (1992) Prevalence of carotid atherosclerosis in diabetic patients. Diabetes Care. 15:1290-1294

39. Langille BL, O'Donell F (1986) Reductions in arterial diameter produced by chronic decreases in blood flow are endothelium-dependent. Science 231:405-407

40. Lemne C, Jogestrand T, Faire U de (1995) Carotid Intima-Media Thickness and plaque in borderline hypertension. Stroke 26:34.39

41. Ludwig M, Kraft K, Rücker W, Hüther AM (1989) Die Diagnose sehr früher arteriosklerotischer Gefäßveränderungen mit Hilfe der Duplexsonographie. Kleine Wochenschr 67:442-446 
42. Ludwig M, Stumpe KO (1994) Karotisultraschall in der Früherkennung der Atherosklerose. Veränderungen der Gefäß-Wanddicke. Dtsch Arzteblatt 91:745-746

43. Ludwig MM, Jörger U, Lund S, Stumpe KO (1993) Divergent responses of common carotid artery intimal-medial thickness to high blood pressure and hypercholesterolemia in relation to age. $\mathrm{J}$ Hypertens. 11:130-131

44. Lynch J, Kaplan GA, Salonen R, Salonen JT (1997) Socioeconomic status and progression of carotid atherosclerosis. Ateriosclerosis, Thrombosis, and Vascular Biology 17:513-519

45. Mannami T, Baba S, Ogata J (2000) Potential of carotid enlargement as a useful indicator affected by high blood pressure in a large general population of a japanese city. Stroke 31:2958-2965

46. Mannami T, Konishi M, Baba S, Nishi N, Terao A (1997) Prevalence of asymptomatic carotid atherosclerosis Lesions detected by high resolution ultrasonography and ist relation to cardiovascular risk factors in the general population of a japanese city. Stroke 28:518-525

47. Markus H, Kapozsta Z, Ditrich R, Wolfe C, Ali N, Powell J, Mendell M, Cullinane M (2001) Increased common carotid intima-media thickness in UK african caribbeans and ist relation to chronic and vascular candidate gene polymorphisms. Stroke 32:24652472

48. Mathur KS, Kashyap SK, Kumar V (1963) Correlation of the extent and severity of atherosclerosis in the coronary and cerebral arteries. Circulation 27:929-934

49. Matthews KA, Owens JF, Kuller LH, Sutton-Tyrrell K, Lassila HC, Wolfson SK (1998) Stress-induced pulse pressure change predicts women's carotid atherosclerosis. Stroke 29:1525-1530

50. Niskanen L, Rauramaa R, Miettinen H, Haffner SM, Mercuri M, Uusitupa M (1996) Carotid artery intima-media thickness in elderly patients with NIDDM and in nondiabetic subjects. Stroke 27:1986-1992 
51. Oberseider J, Felten-Keil G, Schuler K, Goebels N, Hamann GF (1999) Intima-MediaThickness (IMT) bei Patienten mit vertebrobasilären und karotidalen Stenosen/Verschlüssen, Ultraschall in Med. 20:144-149

52. O'Leary DH, Polak JF, Kronmal RA, Kittner SJ, Bond MG, Wolfson SK, Bommer W, Price TR, Gardin JM, Savage PJ (1992) Distribution and correlates of sonographically detected carotid artery disease in the cardiovascular health study. Stroke 23:1752-1760

53. O'Leary DH, Polak JF, Kronmal RA, Manolio TA, Burke GL, Wolfson SK (1999) Carotid-artery intima media thickness as a risk factor for myocardial infarction and stroke in older adults. N Engl J Med. 340:14-22

54. Olsson AG (1991) Regression of femoral atherosclerosis. Circulation 83:698-700

55. O'Rourke M (1990) Arterial Stiffness, systolic blood pressure and logical treatment of Arterial hypertension. Hypertension 15:339-347

56. Pearson TA (1983) Risk factors for arteriographically defined CAD, Baltimore, Md: The John Hopkins University

57. Persson J, Formgren J, Isrealsson B, Berglund G (1994) Ultrasound-determined intimamedia thickness and atherosclerosis. Arterioscler Thromb. 14:261-264

58. Pignoli P, Tremoli E, Poli A, Oreste P, Paoletti R (1986) Intimal plus medial thickness of the arterial wall:a direct measurement with ultrsound imaging. Circulation 74:13991406

59. Polak JF, Kronmal RA, Tell GS, O'Leary DH, Savage PJ, Gardin JM, Rutan GH, Borhani NO (1996) Compensatory increase in common carotid artery diameter. Stroke 27:2012-2015

60. Poli A, Tremoli E, Colombo A, Sirtori M, Pignoli P, Paoletti R (1988) Ultrasonographic measurement of the common carotid artery wall thickness in hypercholesterolemic patients. Athrosclerosis 70:253-261

61. Popele van N, Grobbee DE, Bots ML, Asmar R, Topouchian J, Reneman RS, Hoeks AP, Kuip D van, Hofman A, Witteman J (2001) Association between arterial stiffness and atherosclerosis. Stroke 32:454-460 
62. Pries AR., Secomb TW, Gaehtgens P (1995) Design priciples of vascular beds. Circ Res. 77:1017-1023

63. Pujia A, Rubba P, Spencer MP (1992) Prevalence of extracranial carotid artery disease detectable by echo-doppler in an elderly population. Stroke 23:818-822

64. Riley WA, Barnes RW, Applegate WB, Dempsey R, Hartwell T, Davis VG, Bond MG, Furberg CD (1992) Reproducibility of noninvasive ultrasonic measurement of carotid atherosclerosis. Stroke 23:1062-1068

65. Rosfors S, Hallerstam S, Jensen-Urstad K, Zetterling M, Carlström C (1998) Relationship between intima-media thickness in the common carotid artery and atherosclerosis in the carotid bifurcation. Stroke 29:1378-1382

66. Rubba P, Mercuri M, Faccenda F, Iannuzzi A, Irace C, Strisciuglio P, Gnasso A, Tang R, Andria G, Bond MG, Mancini M (1994) Premature carotid atherosclerosis: Does it occur in both familial hypercholesterolemia and homocystinuria. Stroke 25:934-950

67. Salonen JT, Salonen R (1991) Ultrasonographically assessed carotid morphology and the risk of coronary heart disease. Atheriosclerosis and Thrombosis 11:1245-1249

68. Salonen JT, Salonen R (1993) Ultrasound B-mode imaging in observational studies of atherosclerotic progression. Circulation 87:56-65 (suppl.)

69. Salonen R, Salonen JT (1991) Progression of carotid atherosclerosis and its determinants. Atherosclerosis 81:33-40

70. Schmidt C, Hulthe J, Wikstrand J, Gnarpe H, Gnarpe J, Agewall S, Fagerberg B (2000) Clamydia pneumoniae seropositivity is associated with carotid artery intima-media thickness. Stroke 31:1526-1531

71. Sidhu PS, Desai SR (1997) A simple reproducible method for assessing intimal-medial thickness of the common carotid artery. Brit J Radiology 70:85-89

72. Sitzer M, Puac D, Buehler A, Steckel D, Kegler von S, Markus H, Steinmetz H (2003) Internal carotid artery angle of origin. Stroke 34:950 
73. Solberg LA, Eggen DA (1971) Localization and sequence of developement of atherosclerotic lesions in the carotid and vertebral arteries. Ciculation 43:711-724

74. Stary HC (1987) Macrophages, macrophage foam cells, and eccentric intimal thickening in the coronary arteries of young children. Atherosclerosis 64:91-108

75. Stary HC, Blankenhorn DH, Chandler AB, Glagov S, Insull W, Richardson M, Rosenfeld ME, Schaffer SA, Schwartz CJ, Wagner WD, Wissler RW (1992) A definition of the intima of human arteries and of ist atherosclerosis-prone regions. Circulation Vol. 85, No 1

76. Steinke W, Els T, Hennerici M (1994) Compensatory carotid artery dilatation in early atherosclerosis. Circulation 89:2578-2581

77. Su T, Jeng J, Chien K, Sung F, Hsu H, Lee Y (2001) Hypertension status is the major determinant of carotid atherosclerosis. Stroke 32: 2265

78. Suurküla M, Agewall S, Fagerberg B, Wendelhag I, Wildgren B, Wikstrand J (1994) Ultrasound evaluation of atherosclerotic manifestations in the carotid artery in high-risk hypertensive patients. Arterioscler Thromb. 14:1297-1304

79. Terwey B, Gahbauer H, Montemayor M, Proussalis A, Zöllner G (1984) Die B-BildSonographie der Karotisbifurkation. Ultraschall 5, 190-201

80. Touboul PJ, Prati P, Scarabin PY, Adrai V, Thibout E, Ducimetière P (1992) Use of monitoring software to improve the measurement of carotid wall thickness by B-mode imaging. Journal of hypertension 10:37-41

81. Veller MG, Fisher CM, Nicolaides AN, Renton S, Geroulakos G, Stafford NJ, Sarker A, Szendro G, Belcaro G (1993) Measurement of the ultrasonic intima-media complex thickness in normal subjects. J Vasc Surg 17:719-725

82. Wendelhag I, Wiklund O, Wikstrand J (1992) Arterial wall thickness in familial hypercholesterolemia. Atheriosclerosis and Thrombosis 12:70-77

83. Widder B (1995) Doppler- und Duplex-Sonographie der hirnversorgenden Arterien, 4., überarb. und erw. Aufl.-Berlin: Springer 
84. Wikstrand J, Wendelhag I (1994) Methodological considerations of ultrasound investigation of intima-media thickness and lumen diameter. J Intern Med. 236:555-559

85. Wong M, Edelstein J, Wollman J, Bond MG (1993) Ultrasonic-pathological comparison of the human arterial wall. Arterioscler. \& Thromb. 13:482-486

86. Young W, Gofman JW, Tandy R, Malamud N, Waters ES (1960) The quantitation of atherosclerosis. Am J Cardiol 6:300-308

87. Zarins CK, Giddens DP, Bharadvaj BK, Sottiurai VS, Mabon RF,Glagov S (1983) Carotid bifurcation atherosclerosis. Circ Res. 53:502-514

88. Zureik M, Ducimetière P, Touboul PJ, Courbon D, Bonithon-Kopp C, Berr C, Magne C (2000) Common carotid intima-media thickness predicts occurrence of carotid atherosclerotic plaques. Arteriosclerosis, Thrombosis and Vasc Biol. 20:1622-1630 


\section{Anhang}

Tab. 1 Meßfehler der Dopplerfrequenz bei Berücksichtigung der Blutflußgeschwindigkeit und des Beschallungswinkels $\cos \alpha$

Tab. 2 Intima-Media-Thickness und innerer Querdurchmesser in den Stenosegruppen im Vergleich

Tab. 3 Korrelation nach Pearson zwischen inneren Querdurchmesser und Intima-MediaThickness

Tab. 4 Vergleich der Intima-Media-Thickness nach einfaktorieller ANOVA zwischen den vier Gruppen (1. ohne Stenose/Verschluß, 2. eine Stenose, 3. mindestens zwei Stenosen, 4. Verschluß)

Tab. 5 Intima-Media-Thickness in der A. carotis communis - Vergleich zwischen den Gruppen ohne Stenosen, mit einer Stenose, mit mindestens zwei Stenosen und mit Verschluß

Tab. 6 Intima-Media-Thickness in der A. carotis communis - Vergleich zwischen Männern und Frauen sowie zwischen der rechten und linken Seite

Tab. 7 Innerer Querdurchmesser in der A. carotis communis - Vergleich zwischen Männern und Frauen sowie zwischen der rechten und linken Seite

Tab. 8 Seitenverteilung der Stenosen und Verschlüsse in den hirnversorgenden Arterien

Tab. 9 Intima-Media-Thickness bei Personen mit und ohne kardiovaskulären Risikofaktor im Vergleich

Tab. 10 Korrelation nach Spearman zwischen Alter und Intima-Media-Thickness der A.carotis communis

Tab. 11 Korrelation nach Spearman zwischen Alter und innerem Querdurchmesser der A. carotis communis 


\section{Dank}

An erster Stelle danke ich Herrn Prof. Dr. G. F. Hamann für die Überlassung des Themas und für die Unterstützung während der Durchführung der gesamten Dissertation. Er ermutigte und unterstützte mich zur Veröffentlichung meiner Ergebnisse. Desweiteren gilt mein Dank Herrn Prof. Dr. T. Brandt für die Ermöglichung meiner Arbeit an der Neurologischen Klinik der Ludwig-Maximilians-Universität in München.

Besonders herzlichen bedanken möchte ich mich bei den MTA's des Doppler-Labors, Frau Felten-Keil und Frau Schuler, welche unermüdlich und sehr geduldig an der Durchführung der Arbeit mitwirkten. Nicht zuletzt durch ihre moralische Unterstützung konnte ich immer wieder Mut zur Durchführung dieser Arbeit schöpfen.

Meinem Betreuer, Dr. N. Goebels, möchte ich ebenfalls herzlichen Dank sagen.

Frau Jenny Geiler war stets bester Laune und konnte mich durch ihre temperamentvolle Art immer wieder für neue Ideen inspirieren. Ich danke ihr außerdem für die große Hilfsbereitschaft bei organisatorischen Fragen.

In Zusammenarbeit mit dem Institut für Biometrie im Klinikum Großhadern konnte ich die statistische Auswertung meiner Daten durchführen. Ich danke Herrn Dr. H. Exner und Herrn Kampe für die freundliche Unterstützung.

Für die professionelle Unterstützung von Frau Renate Jahr aus dem LRZ in Sachen Word und Layout möchte ich mich vielmals bedanken.

Last but not least danke ich von Herzen meinen Eltern. Sie haben mir Kraft und Vertrauen zur Fertigstellung dieser Arbeit gegeben. Sie standen mir immer zur Seite. 


\section{Lebenslauf}

Ich wurde am 6. Oktober 1969 in Neuendettelsau/Mittelfranken als Sohn von Marianne und Wilhelm Friedrich Oberseider geboren.

Von 1976 bis 1980 besuchte ich die Grundschule in Sachsen bei Ansbach und von 1980 bis 1989 das Platen-Gymnasium in Ansbach, wo ich im Juni 1989 meine Abiturprüfung ablegte.

Im Frühjahr 1993 begann ich mein Medizinstudium an der Ludwig-MaximiliansUniversität in München.

Als Famulus war ich im Missionskrankenhaus "Church of South India Hospital" im Süden Indiens tätig (1998).

Nach dem 2. Statsexamen (1999) veröffentlichte ich unter meiner Erstautorenschaft die Ergebnisse meiner Doktorarbeit in der Zeitschrift "Ultraschall in der Medizin".

Im Herbst 1999 hatte ich die Gelegenheit im Rahmen des Praktischen Jahres in der chirurgischen Abteilung bei Prof. JP. Chigot im Universitätsklinikum Pitié-Salpêtrière in Paris zu lernen.

Mein Studium beendete ich im Mai 2000 mit dem 3. Staatsexamen an der LudwigMaximilians-Universität.

Von Oktober 2000 bis Juni 2001 abeitete ich als AiP bei Prof. Glasner in der neurologischen Abteilung des Klinikums Neukölln in Berlin. Von Juli 2001 bis Mai 2002 konnte ich mir im Rahmen des ärztlichen Praktikums bei Prof. Mauritz in der Klinik Berlin Kenntnisse in der neurologischen Rehabilitation erwerben.

Am 1. April 2002 wurde mir die Approbation als Arzt erteilt.

Seit April 2002 bin ich im Wenckebach Klinikum in Berlin bei Dr. Trabant in der Abteilung für Psychiatrie und Psychotherapie tätig. 